\title{
Comparison of Strongly Heat-Driven Flow Codes for Unsaturated Media
}

Manuscript Completed: June 1989

Date Published: August 1989

Prepared by

C. D. Updegraff

GRAM, Inc.

1709 Moon Street, N.E.

Albuquerque, NM 87112

Under Contract to:

Sandia National Laboratories

Albuquerque, NM 87185

\section{DISCLAIMER}

This report was prepared as an account of work sponsored by an agency of the United States Government. Neither the United States Government nor any agency thereof, nor any of their employees, makes any warranty, express or implied, or assumes any legal liability or responsibility for the accuracy, completeness, or usefulness of any information, apparatus, product, or process disclosed, or represents that its use would not infringe privately owned rights. Reference herein to any specific commercial product, process, or service by trade name, trademark, manufacturer, or otherwise does not necessarily constitute or imply its endorsement, recommendation, or favoring by the United States Government or any agency thereof. The views and opinions of authors expressed herein do not necessarily state or reflect those of the United States Government or any agency thereof.

\section{Prepared for}

Division of Engineering

Office of Nuclear Regulatory Research

U.S. Nuclear Regulatory Commission

Washington, DC 20555

NRC FIN A1266 


$\cdots, \quad \because \cdots$




\section{DISCLAIMER}

Portions of this document may be illegible in electronic image products. Images are produced from the best available original document. 


\section{ABSTRACT}

Under the sponsorship of the U.S. Nuclear Regulatory Commission, Sandia National Laboratories (SNL) is developing a performance assessment methodology for the analysis of long-term disposal of high-level radioactive waste (HLW) in unsaturated welded tuff. As part of this effort, SNL evaluated existing strongly heat-driven flow computer codes for simulating ground-water flow in unsaturated media. The three codes tested, NORIA, PETROS, and TOUGH, were compared against a suite of problems for which analytical and numerical solutions or experimental results exist. The problems were selected to test the abilities of the codes to simulate situations ranging from simple, uncoupled processes, such as two-phase flow or heat transfer, to fully coupled processes, such as vaporization caused by high temperatures. In general, all three codes were found to be difficult to use because of (1) built-in time stepping criteria, (2) the treatment of boundary conditions, and (3) handling of evaporation/condensation problems. A drawback of the study was that adequate problems related to expected repository conditions were not available in the literature. Nevertheless, the results of this study suggest the need for thorough investigations of the impact of heat on the flow field in the vicinity of an unsaturated HLW repository. Recommendations are to develop a new flow code combining the best features of these three codes and eliminating the worst ones. 


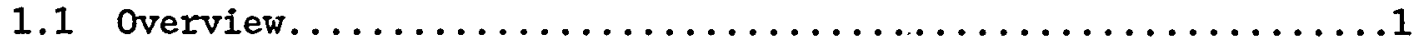

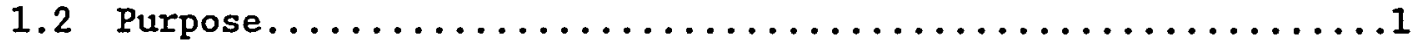

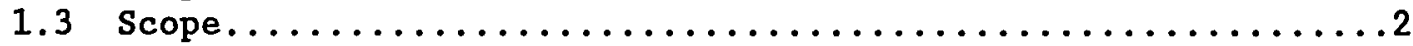

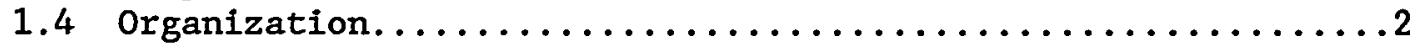

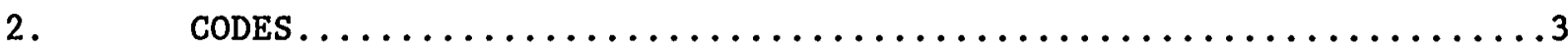

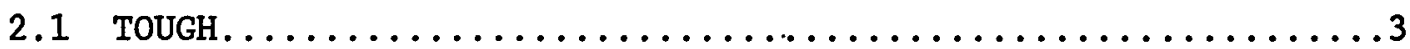

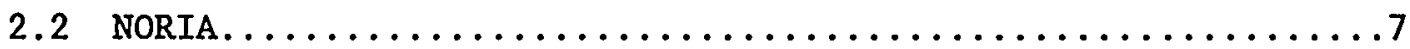

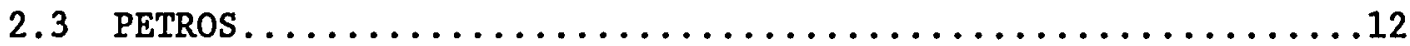

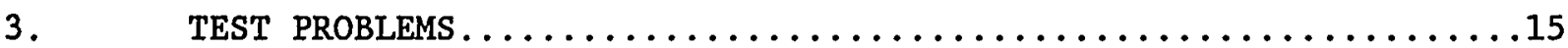

3.1 Test Problems With Exact or Numerical Solutions.........15

3.1.1 One-Dimensional Horizontal Infiltration..........15

3.1 .2 One-Dimensional Heat Transport...............18

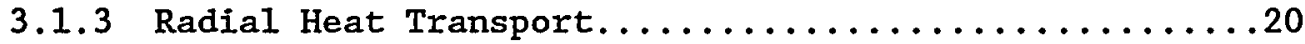

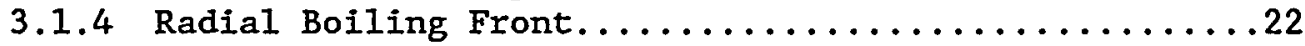

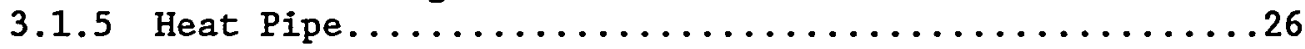

3.2 Test Problems With Experimental Results............... 30

3.2.1 Two-Dimensional Infiltration................

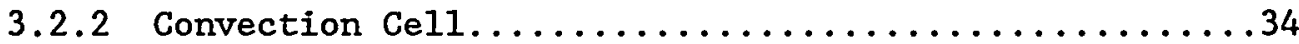

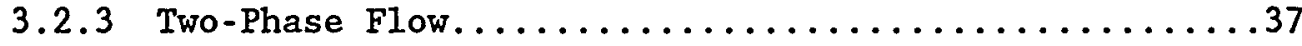

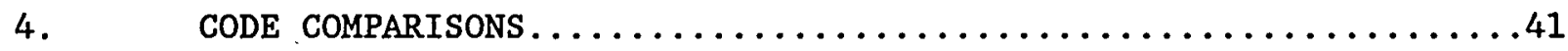

4.1 Comparison with Exact or Numerical Solutions...........41

4.1.1 One-Dimensional Horizontal Infiltration..........41

4.1 .2 One-Dimensional Heat Transport...............49

4.1.3 Radial Heat Transport................... 57

4.1 .4 Radial Boiling Front.......................64

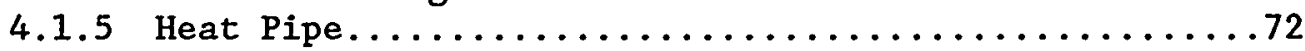

4.2 Comparison with Experimental Results...............78

4.2.1 Two-Dimensional Infiltration...............78

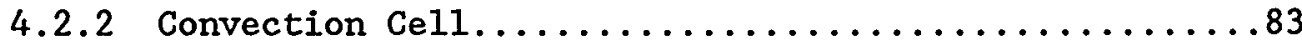

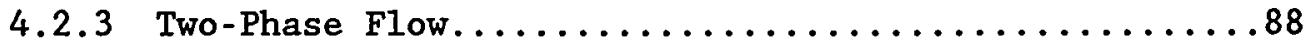


5 .

CONCLUSIONS AND RECOMMENDATIONS................... 97

5.1 Conclusions..........................

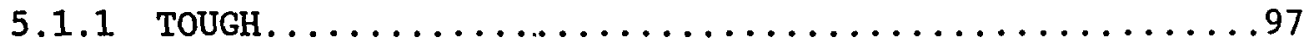

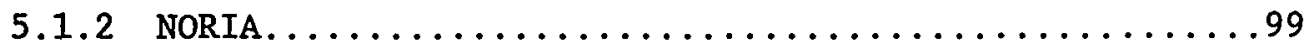

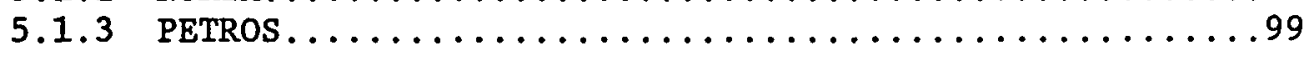

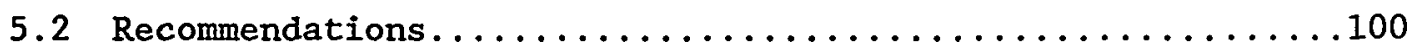

6.

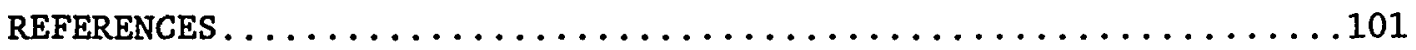




\section{LIST OF FIGURES}

Figure

3.1 One-Dimensional Horizontal Infiltration Problem..............16

3.2 Characteristic Curves for the One-Dimensional Horizontal Infiltration Problem............................... 17

3.3 One-Dimensional Heat Transport Problem...................... 19

3.4 Radial Heat Transport Problem........................21

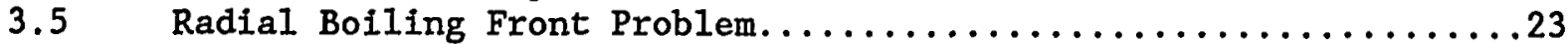

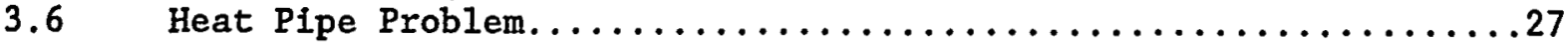

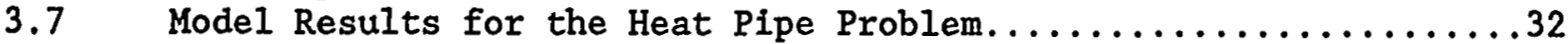

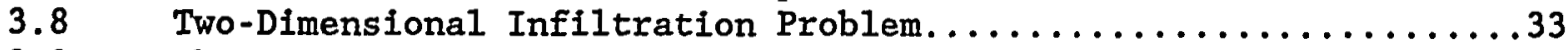

3.9 Characteristic Curves for the Two-Dimensional

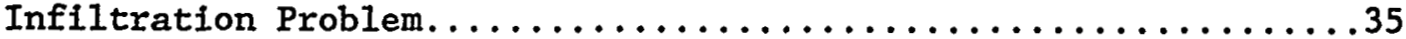

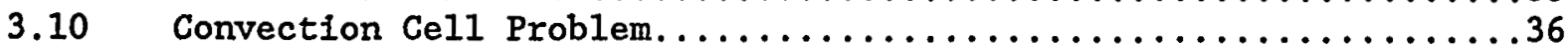

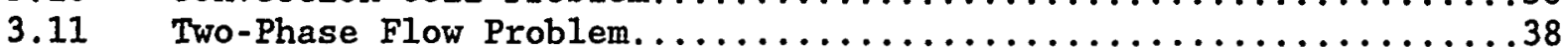

4.1 TOUGH Grid for the One-Dimensional Horizontal

Infiltration Problem.............................. 42

4.2 Comparison of TOUGH Results with Exact Solution for

the One-Dimensional Horizontal Infiltration Problem.........43

4.3 NORIA Grid for the One-Dimensional Horizontal

Infiltration Problem.............................. 45

4.4 Comparison of NORIA Results with Exact Solution

for the One-Dimensional Horizontal Infiltration Problem.......46

4.5 PETROS Grid for the One-Dimensional Horizontal

Infiltration Problem............................47

4.6 Comparison of PETROS Results with Exact Solution

for the One-Dimensional Horizontal Infiltration Problem........48

4.7 TOUGH Grid for the One-Dimensional Heat Transport Problem.......50

4.8 Comparison of TOUGH Results with Exact Solution for the

One-Dimensional Heat Transport Problem.................. 51

4.9 NORIA Grid for the One-Dimensional Heat Transport Problem......52

4.10 Comparison of NORIA Results with Exact Solution for the One-Dimensional Heat Transport Problem.................54

4.11 PETROS Grid for the One-Dimensional Heat Transport Problem......55

4.12 Comparison of PETROS Results with Exact Solution for the One-Dimensional Heat Transport Problem................ 56

4.13 TOUGH Grid for the Radial Heat Transport Problem.............58

4.14 Comparison of TOUGH Results with Exact Solution for the

Radial Heat Transport Problem......................... 59

4.15 NORIA Grid for the Radial Heat Transport Problem............61

4.16 Comparison of NORIA Results with Exact Solution for the

Radial Heat Transport Problem......................62

4.17 PETROS Grid for the Radial Heat Transport Problem...........6. 63

4.18 Comparison of PETROS Results with Exact Solution at $1 \times 10^{6}$ s for the Radial Heat Transport Problem............65

4.19 Comparison of PETROS Results with Exact Solution at $1 \times 10^{9} \mathrm{~s}$ for the Radial Heat Transport Problem............66

4.20 TOUGH Grid for the Radial Boiling Front Problem............67

4.21 Comparison of TOUGH Results with Garg (1980)

Solution for the Radial Boiling Front Problem.............68 
LIST OF FIGURES (continued)

Figure

Page

4.22 PETROS Grid for the Radial Boiling Front Problem...........70

4.23 Comparison of PETROS Results with Garg (1980)

Solution for the Radial Boiling Front Problem.............71

4.24 TOUGH Grid for the Heat Pipe Problem..................73

4.25 Comparison of TOUGH Results with Udell and Fitch (1985)

Steady-State Solution for the Heat Pipe Problem............74

4.26 NORIA Grid for the Heat Pipe Problem..................76

4.27 PETROS Grid for the Heat Pipe Problem....................77

4.28 TOUGH Grid for the Two-Dimensional Infiltration Problem.......79

4.29 Comparison of TOUGH Results with Experimental Results

for the Two-Dimensional Infiltration Problem..............81

4.30 NORIA Grid for the Two-Dimensional Infiltration Problem......82

4.31 TOUGH Grid for the Convection Cell Problem..............84

4.32 Comparison of TOUGH Results with Experimental Results

for the Convection Cell Problem..................... 86

4.33 NORIA Grid for the Convection Cell Problem................87

4.34 TOUGH Grid for the Two-Phase Flow Problem..................89

4.35 Comparison of TOUGH Results with Experimental Results

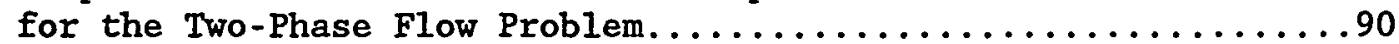

4.36 NORIA Grid for the Two-Phase Flow Problem...............92

4.37 PETROS Grid for the Two-Phase Flow Problem...............94

4.38 Comparison of PETROS Results with Experimental Results

for the Two-Phase Flow Problem....................... 95 
CHAPTER 1

INTRODUCTION

\subsection{Overview}

Welded tuff is now the only potential host rock for the disposal of high-level radioactive waste (HLW) being considered by the U.S. Department of Energy (DOE). The Nuclear Regulatory Commission (NRC) has the responsibility, according to the Nuclear Waste Policy Act of 1982, to regulate DOE's activities pertaining to HLW disposal. One avenue being pursued by NRC to conduct evaluations of DOE's license applications is to develop a performance assessment methodology for analyzing the long-term disposal of HLW in welded tuff. The NRC has contracted to Sandia National Laboratories (SNL) to assist in the development of this methodology. One of the first steps in this work was the evaluation of existing flow codes for unsaturated rock.

The heat generated by an HLW repository can cause a large impact on liquid and gas flow around the repository. For instance, the high heat output of the waste expands the air and water vapor around the repository. The expanding gas creates large gas pressure increases near the repository. This, in turn, forms a pressure gradient in the gas causing it to flow. Because capillary pressure couples the gas pressure to the liquid pressure, a pressure gradient also forms in the liquid and the liquid begins to flow. In addition, if the temperature becomes high enough, water in the rock could vaporize. The water vaporization increases the gas pressure and enhances water flow. Therefore, it is reasonable to expect that the flow field will change after placing HLW in the repository. The standard unsaturated flow codes (Oster, 1982) do not have the capability to model the complex physical situation created by the vaporization of water due to heat loading.

This project originally started as a search for unsaturated flow codes, of which many with varying capabilities exist. Unfortunately, few can model heat transport and, if they do have this capability, they do not include vaporization of water. While SNL searched for unsaturated flow codes, the interest in modeling strongly heat-driven flow became apparent. The modeling of strongly heat-driven flow is applicable not only to HLW disposal but to geothermal resources as well. TOUGH, a strongly heat-driven flow code which has some application to HLW problems, was developed initially for geothermal reservoirs. Because of the recent development of the strongly heat-driven flow codes, SNL decided to evaluate them for possible use in the tuff methodology.

\subsection{Purpose}

The computer codes evaluated in this report may have limitations with respect to the development of aspects of the tuff methodology. This study attempts to identify these limitations and to suggest ways to overcome them. These 
suggestions may range from modifying an existing code to developing an entirely new one.

\subsection{Scope}

The investigation proceeded in a sequential manner, which the organization of this report reflects. First, SNL searched the literature to find strongly heat-driven flow codes from a list of available unsaturated flow codes. After reviewing the codes to determine their capabilities, SNL searched the literature to find problems to test these capabilities. The problems tested such capabilities as heat transport, unsaturated flow, and vaporization of water. Then, SNL examined each code's capabilities by modeling each of the test problems. The results of the modeling formed the basis for recommending code improvements or designing a new code.

\subsection{Organization}

This report consists of six sections divided according to the work involved in studying the strongly heat-driven flow computer codes. Section 1 describes the underlying reasons for choosing the strongly heat-driven flow codes over the standard unsaturated flow codes. Section 2 presents a description of the three strongly heat-driven flow codes evaluated by simulating the test problems. This section explains the physical processes, boundary conditions, numerics, limitations, and structure of the codes. Section 3 presents a list of the test problems selected for the evaluation. These problems include five for which exact or numerical solutions exist and three for which experimental results exist. Section 4 presents the results of running the three codes and comparing them to the exact and numerical solutions, and experimental results. Section 5 presents a list of recommendations to consider for developing a new strongly heat-driven flow code. Section 6 presents a list of references used for describing the codes and the problems.

Several sections of this report refer to gas and liquid. In this report, when the codes track the mass of several components, the gas phase consists of water vapor and air. If the codes model only one component, the gas phase consists of either air or water vapor. Although air and water vapor are gases, this report refers to them by their respective names. Liquid refers to any liquid, but in this report it means water.

When modeling a problem, the code input data were kept as similar as possible for each code. For instance, the grid spacing for a problem was kept the same as much as possible. This presented assurances that the codes be equitably compared for results and running times. All problems were run on SNL's CRAY XMP/24 computer. 
CHAPTER 2

CODES

Several computer codes capable of simulating strongly heat-driven flow problems were found. These codes include TOUGH, developed by Pruess (1987) at Lawrence Berkeley Laboratories; NORIA, developed by Bixler (1985) at Sandia National Laboratories; PETROS, developed by Hadley (1985) at Sandia National Laboratories; and WAFE, developed by Travis at Los Alamos National Laboratory (Oster, 1982). All of these codes, except WAFE, have published documentation. Because WAFE was undocumented, it was neither reviewed nor tested in this report.

\subsection{TOUGH}

TOUGH (Pruess, 1987) solves the gas- and liquid-phase flow of air and water, and heat transport in a fully coupled way. The formulation used in TOUGH is analogous to that used in multiphase, multicomponent geothermal or steam-flooded hydrocarbon reservoir problems. The governing fluid flow equations account for gaseous diffusion, Darcy flow, capillary pressure, vaporization, and condensation. Vaporization and condensation with latent heat effects, and conduction and convection of heat are included in the energy equation. Water, air, and rock are assumed to be in thermodynamic equilibrium at all times. The flow domain can include liquid-, gas-, and two-phase regions, indicating that the code handles both saturated and unsaturated flow problems either individually or simultaneously. The thermophysical properties of liquid and vaporized water are represented by the International Formulation Committee's (1967) steam tables. Air is approximated as an ideal gas and additivity of partial pressures is assumed for air-water vapor mixtures.

TOUGH solves three nonlinear partial differential equations simultaneously. These consist of the conservation equations for air, water, and energy.. Air and water can be transported in either the liquid phase, the gas phase, or both. The dissolution of air in water is represented by Henry's law and flow by Darcy's law.

The code can simulate flow in one, two, or three dimensions because the method of solution is based on a general integrated finite-difference method. Time stepping is accomplished by a fully implicit procedure. The resulting nonlinear difference equations are linearized by the Newton-Raphson technique. The linearized equations are solved by the Harwell MA28 matrix solver (Duff, 1977) which stores only the nonzero elements of a matrix thus reducing core storage requirements for the code.

The governing flow equations used in TOUGH are similar to those used in modeling geothermal reservoirs. The governing mass conservation equation of water can be written as:

$$
\frac{\partial}{\partial t} \int_{V_{n}} M^{W} d v=\int_{\Gamma_{n}} F^{W} \cdot n d \Gamma+\int_{V_{n}} q^{W} d v
$$


where $\mathrm{M}^{\mathrm{W}}$ is the mass of water per unit volume in the integrated finite-difference grid block, $V_{n}$ is the volume of the grid block, $F^{W}$ is the mass flux of water out the grid block, $\Gamma_{n}$ is the surface area of a grid block, $n$ is an outward pointing unit normal vector, and $q^{W}$ is the mass production of water per unit volume. Note that water is stored in both the liquid and gas phases in the above equation. The mass of water per unit volume is, therefore,

$$
M^{W}=\phi\left(S_{1} \rho_{1} X^{W}+S_{g} \rho_{g} X_{g}^{W}\right)
$$

where $\phi$ is the matrix porosity, $s_{1}$ is the liquid saturation, $\rho_{1}$ is the liquid density, $\mathrm{X}^{\mathrm{w}}{ }_{1}$ is the mass fraction of water in the liquid, $S_{g}$ is the gas saturation $\left(1-S_{1}\right), \rho_{g}$ is the gas density, and $x_{g}^{W}$ is the mass fraction of water in the gas.

A set of equations, similar to the ones for water, can be written for air:

$$
\frac{\partial}{\partial t} \int_{V_{n}} M^{a} d v=\int_{\Gamma_{n}} F^{a} \cdot n d \Gamma+\int_{V_{n}} q^{a} d v
$$

and

$$
M^{a}=\phi\left(S_{1} \rho_{1} X_{1}^{a}+S_{g} \rho_{g} X_{g}^{a}\right)
$$

where $\mathrm{M}^{\mathrm{a}}$ is the mass of air per unit volume in a grid block, $\mathrm{F}^{\mathrm{a}}$ is the mass flux of air out the grid block, $q^{a}$ is the mass production of air per unit volume, $x^{2}$ is the mass fraction of air in the liquid and $x_{g}^{2}$ is the mass fraction of air in the gas.

The mass flux for either the air or water component is simply the sum of the mass fluxes of a component over both phases. For water the mass flux is:

$$
F^{W}=F_{1}^{W}+F_{g}^{W}
$$

and for air:

$$
F^{a}=F_{1}^{a}+F_{g}^{a}
$$

where $F^{W}$ is the mass flux of water in the liquid phase, $F^{W}$ is the mass flux of water in the gas phase, $F^{2}{ }_{1}$ is the mass $f 1$ ux of air in the liquid phase, and $\mathrm{F}_{\mathrm{g}}^{\mathrm{a}}$ is the mass flux of air in the gas phase. The mass flux of each component in a phase is governed by Darcy's law and gaseous diffusion. These four equations are: 


$$
\begin{aligned}
& F_{1}^{W}=-k \frac{k_{r 1}}{\mu_{1}} \rho_{1} X^{W}{ }_{1}\left(\nabla P_{1}-\rho_{1} g\right) \\
& F_{1}^{a}=-k \frac{k_{r 1}}{\mu_{1}} \rho_{1} X^{a}{ }_{1}\left(\nabla P_{1}-\rho_{1} g\right) \\
& F_{g}^{W}=-k \frac{k g}{\mu_{g}} \rho_{g} X_{g}^{W}\left(\nabla P_{g}-\rho_{g} g\right)-D_{v a} \rho_{g} \nabla X_{g}^{W}
\end{aligned}
$$

and

$$
F_{g}^{a}=-k \frac{k_{r g}}{\mu_{g}} \rho_{g} x_{g}^{a}\left(\nabla P_{g}-\rho_{g} g\right)-D_{v a} \rho_{g} \nabla x_{g}^{a}
$$

where $k$ is the porous medium permeability in the direction of $n, k_{r l}$ is the liquid relative permeability, $\mathrm{k}_{\mathrm{rg}}$ is the gas relative permeability, $\mu_{1}$ is the liquid dynamic viscosity, ${ }_{\mu} g$ is the gas dynamic viscosity, $\mathrm{P}_{1}$ is the liquid pressure, $\mathrm{P}_{\mathrm{g}}$ is the gas pressure, $\mathrm{g}$ is the acceleration of gravity and $D_{v a}$ is the Binary diffusion coefficient for air-water vapor mixtures. TOUGH cannot handle the off-diagonal terms of the permeability tensor. Further, the code allows only functions to determine the relationship between relative permeability and saturation, and contains a library of functions for these in one of its subroutines. The solubility of air in the water is governed by Henry's law:

$$
\mathrm{X}_{1}^{\mathrm{a}}=\frac{\mathrm{P}_{\mathrm{a}}}{\mathrm{K}_{\mathrm{h}}} \frac{\mathrm{MW} \mathrm{a}}{\mathrm{MW}}
$$

where $P_{a}$ is the air partial pressure, $K_{h}$ is Henry's constant, $M W_{a}$ is the molecular weight of air, and $\mathrm{MW}_{\mathrm{w}}$ is the molecular. weight of water. The binary diffusion coefficient is dependent on both temperature and pressure and is written:

$$
D_{v a}=\tau \phi S \frac{D_{v a}^{\circ}}{P_{g}}\left[\frac{T-273^{\circ} .15}{273.15}\right]^{\eta}
$$

where $T$ is tortuosity, $T$ is temperature, and $D^{\circ}$ va and $\eta$ are parameters which have values of $2.13 \times 10^{-5} \mathrm{~m}^{2} / \mathrm{s}$ and 1.80 , respectively, for a pressure of one bar and a temperature of $0^{\circ} \mathrm{C}$. Partial pressures are assumed to be additive to determine the gas pressure:

$$
P_{g}=P_{a}+P_{v}
$$


where $P_{a}$ is the air partial pressure and $P_{v}$ is the vapor partial pressure.

The energy conservation equation can be written in an integral form similar to the air and water conservation equations. The energy equation is:

$$
\frac{\partial}{\partial t} \int_{V_{n}} M^{h} d v=\int_{\Gamma_{n}} F^{h} \cdot n d \Gamma+\int_{V_{n}} q^{h} d v
$$

where $M^{h}$ is the amount of heat per unit volume in an integrated finite difference grid block, $F^{h}$ is the heat flux out the grid block and $q^{h}$ is the amount of heat produced per unit volume of grid block. The heat term contains contributions from both the rock, liquid, and vapor:

$$
M^{h}=(1-\phi) \rho_{r} c_{r} T+\phi\left(S_{1} \rho_{1} u_{1}+S_{g} \rho_{g} u_{g}\right)
$$

where $\rho_{r}$ is the rock grain density, $c_{r}$ is rock grain specific heat, $u_{1}$ is the specific internal energy of the liquid, and $u_{g}$ is the specific internal energy of the gas. The heat flux term consists of conductive and convective parts:

$$
F^{h}=-K \nabla T+h^{W}{ }_{1} F^{W}{ }_{1}+h^{a}{ }_{1} F^{a}{ }_{1}+h^{w}{ }_{g} F^{W}{ }_{g}+h^{a}{ }_{g} F_{g}^{a}
$$

where $K$ is the thermal conductivity of the rock-fluid mixture, $h^{w}$ is the specific enthalpy of water in the liquid phase, $h^{a}{ }_{1}$ is the specific enthalpy of air in the liquid phase, $h^{\mathrm{w}}$ is the specific enthalpy of water in the gas phase, and $\mathrm{h}^{\mathrm{a}}$ is the specific enthalpy of air in the gas phase. The thermal conductivity model, selected by the user, is allowed to vary with saturation by either of the following equations:

$$
\mathrm{k}=\mathrm{k}_{\mathrm{dry}}+\sqrt{\mathrm{s}_{1}}\left(\mathrm{k}_{\mathrm{wet}}-\mathrm{k}_{\mathrm{dry}}\right)
$$

and

$$
\mathrm{K}=\mathrm{k}_{\mathrm{dry}}+\mathrm{s}_{1}\left(\mathrm{k}_{\mathrm{wet}}-\mathrm{k}_{\mathrm{dry}}\right)
$$

where $K_{d r y}$ is the thermal conductivity of the totally dry porous medium and $\mathrm{K}_{\text {wet }}$ is the thermal conductivity of the fully saturated porous medium.

The above equations are recast into an integrated finite-difference form for solution. The integrated finite-difference method employed in TOUGH is general. It can be used to solve problems in one, two or three dimensions and Cartesian or axisymmetric geometries. Because of this general method, the TOUGH code requires volumes for the grid blocks, distances between the 
grid blocks, and interfacial areas between the grid blocks as input data instead of grid-block spacings that might be required by finite-element or node-centered finite-difference codes. Because of its generality, TOUGH is not limited to rectangular or rectangular-parallelpiped grid blocks. The grid blocks can be any shape such as pentagons or tetrahedrons, but the aspect ratio must not be extreme.

The recast equations are differenced backwards in time and linearized with the Newton-Raphson procedure. The resulting algebraic equations are solved with the Harwell matrix inverter (Duff, 1977) until the solution converges.

TOUGH handles several types of boundary conditions. If a grid block edge is . not connected to another grid block, then that edge becomes a zero flux boundary condition for all the dependent variables. If a grid block is assigned an extremely large volume relative to the other grid blocks, then the dependent variables are essentially fixed with respect to time. Time varying flux boundary conditions can be implemented by using the time varying source/sink capability in TOUGH. However, time varying Dirichlet type boundary conditions have to be implemented using TOUGH'S restart capability, a process which can be cumbersome in practice.

TOUGH's method of handling boundary conditions may cause difficulty in actually implementing them. For instance, suppose a problem requires a zero liquid flux and constant temperature boundary condition along a grid block edge. The first condition implies that the grid block is not connected to another grid block through the boundary edge. The second condition implies that the grid block is connected to a large volume grid block through the boundary edge. Clearly, these two condition are incompatible. However, with some ingenuity these difficulties in implementing boundary conditions can be overcome. The important point to remember is that implementing boundary conditions with TOUGH is not always straightforward.

\subsection{NORIA}

NORIA (Bixler, 1985) is designed to simulate liquid, vapor, air, and energy transport in partially saturated and saturated porous media. The following mechanisms are included in NORIA: (1) transport of water, vapor, and air due to pressure gradients; (2) transport of water, vapor, and air due to density gradients; (3) binary diffusion of vapor and air; (4) Knudsen diffusion of vapor and air; (5) thermodiffusion of vapor and air; (6) conduction of sensible heat; (7) convection of sensible heat; (8) evaporation and condensation; (9) nonequilibrium and equilibrium vapor pressure models; and (10) capillary pressure. The user can define nearly all the code's thermodynamic and constitutive properties in terms of the remaining dependent or independent variables.

NORIA solves four nonlinear partial differential equations governing the flow of water, vapor, air, and energy. These equations consist of a water pressure equation, a vapor partial pressure equation, an air partial pressure equation, and a heat equation. The equations are solved by the Galerkin finite-element method. Time stepping is accomplished by a two-step time integrator with automatic time-step selection. The nonlinear difference 
equations formed by application of the finite-element method are solved simultaneously by Newton-Raphson iteration. Normally, a one-step iteration is used; however, a multistep iteration is used if the correction on the first iteration is larger than a specified amount.

NORIA solves three mass conservation equations and a heat conservation equation. The liquid conservation equation is:

$$
\rho_{1} \frac{\partial \theta}{\partial t}=-\nabla \cdot j_{1}-F_{v}
$$

where $\rho_{1}$ is the liquid density, $\theta$ is the soil/rock moisture content, $j_{l}$ is the liquid mass flux vector, $F_{v}$ is the vaporization rate of water per unit volume and $t$ is time. Note that the liquid density is constant. The liquid mass flux is governed by Darcy's law and the Boussinesq approximation for natural convection due to temperature gradients:

$$
\mathbf{j}_{1}=-\rho_{1} \frac{\underline{\mathbf{k}} \mathbf{k}_{\mathrm{I}}}{\mu_{1}} \cdot \nabla\left(P-\rho_{1} \mathrm{~g}_{\mathrm{z}} \mathrm{Bz} \Delta \mathrm{T}\right)
$$

where $\underline{k}$ is the porous media permeability tensor, $k_{r l}$ is the liquid relative permeability, $\mu_{1}$ is the liquid dynamic viscosity, $P$ is the effective pressure, $g_{z}$ is the gravitational constant, $B$ is the liquid volumetric expansion coefficient, $z$ is the vertical coordinate, and $\Delta T$ is the difference between the local temperature and a reference temperature. The effective pressure, $P$, is a combination of a liquid pressure term and an elevation term:

$$
P=P_{1}+\rho_{1} g_{z} z
$$

where $P_{1}$ is the pressure in the liquid. The vapor conservation equation is written as:

$$
\frac{\partial}{\partial t}(\phi-\theta) \rho_{\mathrm{v}}=-\nabla \cdot \mathbf{j}_{\mathrm{v}}+\mathrm{F}_{\mathrm{v}}
$$

where $\rho_{\mathrm{V}}$ is the vapor density and $\mathrm{j}_{\mathrm{V}}$ is the vapor mass flux vector. The vapor density is governed by the ideal gas law:

$$
\rho_{v}=\frac{P_{v}}{R_{v} T}
$$

where $P_{v}$ is the vapor partial pressure and $R_{v}$ is the ideal gas constant divided by the molecular mass of the vapor. The mass flux term is controlled 
by several factors including Darcy's law, natural convection caused by temperature and pressure gradients, Knudsen diffusion, binary diffusion, and thermodiffusion:

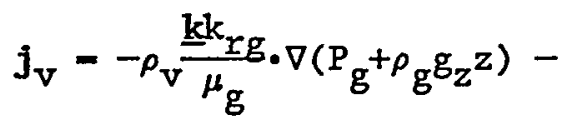

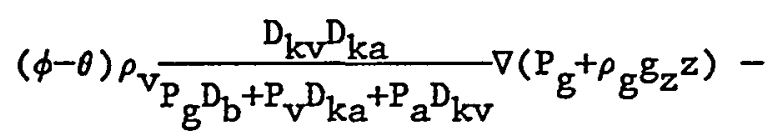

$$
\begin{aligned}
& (\phi-\theta) \rho_{v_{\mathrm{P}_{\mathrm{g}}} \mathrm{D}_{\mathrm{b}}+\mathrm{P}_{\mathrm{v}} \mathrm{D}_{\mathrm{ka}}+\mathrm{P}_{\mathrm{a}} \mathrm{D}_{\mathrm{kv}}} \nabla\left(\mathrm{P}_{\mathrm{v}}+\rho_{\mathrm{v}} \mathrm{g}_{\mathrm{z}} z\right)+ \\
& (\phi-\theta) \rho \frac{\mathrm{R}_{\mathrm{a}} \mathrm{D}_{\mathrm{kv}} \mathrm{D}_{\mathrm{t}}}{\mathrm{g}_{\mathrm{g}} \mathrm{D}_{\mathrm{b}}+\mathrm{P}_{\mathrm{v}} \mathrm{D}_{\mathrm{ka}}+\mathrm{P}_{\mathrm{a}} \mathrm{D}_{\mathrm{kv}}} \nabla \mathrm{T}
\end{aligned}
$$

where $k_{r g}$ is the relative gas permeability, $\mu_{\mathrm{g}}$ is the gas dynamic viscosity, $\mathrm{P}_{a}$ is the air partial pressure, $\mathrm{P}_{\mathrm{g}} \mathrm{g}_{\text {is }}$ the gas pressure $\left(\mathrm{P}_{\mathrm{a}}+\right.$ $\left.\mathrm{P}_{\mathrm{v}}\right), \rho_{\mathrm{g}}$ is the gas density $\left(\rho_{\mathrm{a}}+\rho_{\mathrm{v}}\right), \mathrm{D}_{\mathrm{kv}}$ is ${ }^{\mathrm{g}}$ the vapor Knudsen diffusion coefficient, $D_{k a}$ is the air Knudsen diffusion coefficient, $D_{b}$ is the binary diffusion coefficient and $D_{t}$ is the thermodiffusion coefficient. The terms on the right-hand side of the above equation represent, in order, Darcy flow from pressure and density changes, Knudsen diffusion, binary diffusion, and thermodiffusion.

A similar set of equations as described in the preceding paragraphs can be developed for the air phase. The main difference is that there is no vaporization term in the air continuity equation. Therefore, the continuity equation for air is:

$$
\frac{\partial}{\partial t}(\phi-\theta) \rho_{a}=-\nabla \cdot j_{a}
$$

where $\rho_{a}$ is the air density and $j_{a}$ is the air mass flux vector. The air density is determined from the ideal gas law:

$$
\rho_{a}=\frac{P_{a}}{R_{a} T}
$$

where $R_{a}$ is the ideal gas constant divided by the molecular mass of air. The expression for the air mass flux vector is:

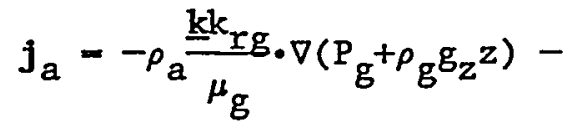

$$
\begin{aligned}
& (\phi-\theta) \rho \frac{D_{k a} D_{k v}}{P_{g} D_{b}+P_{v} D_{k a}+P_{a} D_{k v}} \nabla\left(P_{g}{ }^{+} \rho_{g} g_{z} z\right)-
\end{aligned}
$$




$$
\begin{aligned}
& (\phi-\theta) \rho \frac{\left(\mathrm{P}_{\mathrm{g}} / \mathrm{P}_{\mathrm{a}}\right) \mathrm{D}_{\mathrm{ka}} \mathrm{D}_{\mathrm{b}}}{\mathrm{P}_{\mathrm{g}} \mathrm{D}_{\mathrm{b}}+\mathrm{P}_{\mathrm{v}} \mathrm{D}_{\mathrm{ka}}+\mathrm{P}_{\mathrm{a}} \mathrm{D}_{\mathrm{kv}}} \nabla\left(\mathrm{P}_{\mathrm{a}}+\rho_{\mathrm{a}} \mathrm{g}_{\mathrm{z}} \mathrm{z}\right)- \\
& (\phi-\theta) \rho_{\mathrm{g}_{\mathrm{g}} \mathrm{D}_{\mathrm{b}}+\mathrm{P}_{\mathrm{v}} \mathrm{D}_{\mathrm{ka}}+\mathrm{P}_{\mathrm{a}} \mathrm{D}_{\mathrm{kv}}} \nabla \mathrm{T} .
\end{aligned}
$$

The energy conservation equation is based on thermal equilibrium between the arious phases and components, the convective transport of heat, the conductive transport of heat, the heat of vaporization, and the addition of heat. These factors lead to the heat equation:

$$
[\rho c]_{a v e} \frac{\partial T}{\partial t}=-\left(c_{1} j_{1}+c_{v} j_{v}+c_{a} j_{a}\right) \cdot \nabla T+\nabla \cdot(\underline{\Lambda} \cdot \nabla T)-F_{v} L+Q
$$

where

$$
[\rho \mathrm{c}]_{\text {ave }}=(1-\phi) \rho_{\mathrm{r}} \mathrm{c}_{\mathrm{r}}+\theta \rho_{1} \mathrm{c}_{1}+(\phi-\theta)\left(\rho_{\mathrm{v}} \mathrm{c}_{\mathrm{v}}+\rho_{\mathrm{a}} \mathrm{c}_{\mathrm{a}}\right)
$$

and $\rho_{r}$ is the rock grain density, $c_{r}$ is the rock grain specific heat, $c_{1}$ is the liquid specific heat, $c_{\mathrm{v}}$ is the vapor specific heat, $c_{a}$ is the air specific heat, $\underline{\Lambda}$ is the thermal conductivity tensor, $L$ is the latent heat of vaporization per unit mass, and $Q$ is the heat input per unit volume.

A nonequilibrium vapor pressure model is implemented in NORIA as opposed to the equilibrium model contained in TOUGH. In an equilibrium model, the vapor is always at its equilibrium pressure for the local temperature (i.e., in thermodynamic equilibrium with the liquid). The nonequilibrium model depends on the vaporization rate. The default vaporization model implemented in NORIA is :

$$
F_{v}=C \frac{\left(\theta-\theta_{r}\right)}{P_{v}{ }^{x}-P_{v}}
$$

where $\theta_{\mathrm{r}}$ is a residual moisture content, $\mathrm{P}_{\mathrm{v}}{ }^{*}$ is the equilibrium vapor pressure of water as a function of temperature, and $C$ is a constant of proportionality. By setting $C$ to a large value an equilibrium model can be implemented. However, when $\theta$ approaches $\theta_{r}$, the rate of vaporization approaches zero in a smooth manner.

The water, vapor, air, and porous media hydrologic and thermal properties are implemented as default models in subroutines. These subroutines can be easily changed to reflect different property models. As a matter of fact, NORIA is not necessarily limited to water, vapor, and air. By changing the models implemented in the subroutines, NORIA can model almost any vaporizable liquid and noncondensable gas.

The above conservation and flux equations are solved by means of the Galerkin finite-element method. The basis functions used in NORIA are quadratic 
functions applied to either subparametric or isoparametric elements. The subparametric element sides are described by polynomials whose highest order is less than the highest order of the basis functions. In NORIA, the sides of the subparametric elements are described by linear functions. The isoparametric elements are described by polynomials of the same order as the basis functions, that is, quadratic functions.

The resulting difference equations are solved in time by a two-step, predictor-corrector method. In the first step the dependent variables are estimated (or predicted) at the next time step by a second-order Adams-Bashford predictor:

$$
y^{p_{n+1}}=y_{n}+\frac{\Delta t_{n}}{2}\left\{\left[2+\frac{\Delta t_{n}}{\Delta t_{n-1}}\right] \dot{y}_{n}-\frac{\Delta t_{n}}{\Delta t_{n-1}} \dot{y}_{n-1}\right\}
$$

where $y^{p}{ }_{n+1}$ is the predicted value of the dependent variable at the new time, $y_{\mathfrak{n}}$ is the value of the dependent variable at the present time, $\dot{y}_{\mathfrak{n}}$ is the rate of change of the dependent variable at the present time, $\dot{y}_{n-1}$ is the rate of change of the dependent variable at the preceding time, $\Delta t_{n}$ is the present time-step size $\left(t_{n+1}-t_{n}\right)$, and $\Delta t_{n-1}$ is the preceding time-step size $\left(t_{n}-t_{n-1}\right)$. The rate of change of the dependent variables are described by:

$$
\dot{y}_{n}=\frac{2}{\Delta t_{n-1}}\left[y_{n}-y_{n-1}\right]-\dot{y}_{n-1}
$$

and

$$
\dot{y}_{n-1}=\frac{\Delta t_{n-2}}{\Delta t_{n-1}+\Delta t_{n-2}}\left[\frac{y_{n}-y_{n-1}}{\Delta t_{n-1}}\right]+\frac{\Delta t_{n-1}}{\Delta t_{n-1}+\Delta t_{n-2}}\left[\frac{y_{n-1}-y_{n-2}}{\Delta t_{n-2}}\right] .
$$

By describing rates of change this way, Bixler (1985) found that temporal oscillations in the solution are eliminated as steady state is approached. It can be seen that the above predictor is an explicit scheme, but because it requires values of the dependent variable from two preceding time steps, the scheme cannot be used on the first two time steps. Instead, the equations for the first two time steps are solved by a backward difference or fully implicit procedure.

Once the predicted solution is calculated, it is improved with a trapezoid-rule corrector. The corrector produces highly nonlinear algebraic equations which are solved by a Newton iteration procedure. Generally, only one Newton iteration is required but, if the difference between the predicted and corrected solution is large, up to three Newton iterations may be necessary.

NORIA also has a capability for time-step-size adjustment after the first predictor-corrector time step (third time step overal1). The criteria is based on differences between the predicted and corrected solutions. 
Both Dirichlet and Neumann boundary conditions can be implemented in NORIA. The code has capabilities to handle both fixed and time-varying dependent variables, and constant and time-varying fluxes. Zero flux boundary conditions are the default boundary conditions in NORIA.

\section{$2.3 \quad$ PETROS}

PETROS is designed to simulate problems similar to those simulated by NORIA. PETROS solves the same number and types of nonlinear equations and handles the same physical processes as NORIA but in a slightly different manner. The main difference between the two codes is that PETROS solves only one-dimensional problems, either in linear, radial, or spherical coordinates, and solves the equations with the finite-difference method. There are also some differences between the codes in the way the time integrations are performed. PETROS uses a modified version of the time integrator in NORIA. In addition, the format for data input in the PETROS code is not as general as that in NORIA.

PETROS solves the same three mass conservation equations and heat conservation equation as NORIA. However, the liquid conservation equation in PETROS is formulated with respect to saturation rather than pressure as in NORIA. The liquid mass conservation equation is:

$$
\phi \rho_{1} \frac{\partial S}{\partial t}+\frac{I}{r^{i}} \frac{\partial}{\partial r}\left[r^{i_{j}}\right]=-F_{v}
$$

where $\phi$ is porosity, $\rho_{1}$ is liquid density, $S$ is saturation, $r$ is distance, $t$ is time, $j_{1}$ is liquid mass flux, $F_{v}$ is liquid evaporation rate per unit volume, and $i$ is either 1, 2, or 3 for linear, radial, or spherical geometry, respectively. The liquid mass flux is:

$$
j_{1}=-\rho_{1} \frac{k_{r l} 1}{\mu_{1}}\left[\frac{\partial P_{1}}{\partial r}-\rho_{1} g_{r}\right]
$$

where $\mathrm{k}$ is the porous media permeability, $\mathrm{k}_{\mathrm{rl}}$ is the liquid relative permeability, $\mu_{1}$ is the liquid dynamic viscosity, and $g_{r}$ is the gravitational constant in the direction of positive $x$.

The vapor mass conservation equation is written as:

$$
\frac{1}{r^{i}} \frac{\partial}{\partial r}\left[r^{i} j_{v}\right]=F_{v}
$$

where $j_{\mathrm{v}}$ is the vapor mass flux. Note that this equation is treated as a steady-state equation. The vapor mass flux is controlled by the effects of Darcy's law, Knudsen diffusion, and binary diffusion: 


$$
j_{v}=-\frac{\phi(1-S) m_{v} D_{k v}}{\kappa T}\left\{\frac{\frac{\partial P_{a}}{\partial r}+\left[1+\frac{P_{g} \lambda D_{b}}{P_{v} D_{k v}}\right] \frac{\partial P_{v}}{\partial r}}{1+\frac{P_{a} \lambda}{P_{v}}+\frac{P_{g} \lambda D_{b}}{P_{v} D_{k v}}}\right\}-\frac{k_{r g} m_{v} P_{v}}{\mu_{g} \kappa T} \frac{\partial P_{g}}{\partial r}
$$

where $D_{k v}$ is the vapor Knudsen diffusion coefficient, $D_{b}$ is the binary diffusion coefficient, $\mathrm{m}_{\mathrm{v}}$ is the mass of a water molecule, $\kappa$ is Boltzmann's constant, $\mathrm{P}_{\mathrm{a}}$ is the air partial pressure, $\mathrm{P}_{\mathrm{v}}$ is the vapor partial pressure, $P_{g}$ is the gas pressure $\left(P_{a}+P_{v}\right), \lambda$ is the square root of the mass ratio of air to water, $k_{\mathrm{g}}$ is the gas relative permeability, $\mu_{g}$ is the gas dynamic viscosity, and $T$ is temperature.

The air mass conservation equation is similar to the vapor mass conservation except for the evaporation term:

$$
\frac{1}{r^{i}} \frac{\partial}{\partial r}\left[r^{i_{j}}\right]=0
$$

where $j_{a}$ is the air mass flux. The air mass flux equation is similar to the vapor mass flux equation:

$$
\mathrm{j}_{\mathrm{a}}=-\frac{\phi(1-\mathrm{S}) \mathrm{m}_{\mathrm{v}} \lambda \mathrm{D}_{\mathrm{kv}}}{\kappa \mathrm{T}}\left\{\frac{\frac{\partial \mathrm{P}_{\mathrm{v}}}{\partial r}+\left[1+\frac{\mathrm{P}_{\mathrm{g}} \mathrm{D}_{\mathrm{b}}}{\mathrm{P}_{\mathrm{a}} \mathrm{D}_{k v}}\right] \frac{\partial \mathrm{P}_{\mathrm{a}}}{\partial r}}{1+\frac{\mathrm{P}_{\mathrm{v}} \lambda}{\mathrm{P}_{\mathrm{a}}}+\frac{\mathrm{P}_{\mathrm{g}} \mathrm{D}_{\mathrm{b}}}{\mathrm{P}_{\mathrm{a}} \mathrm{D}_{k v}}}\right\}-\frac{k_{\mathrm{rg}} \mathrm{m}_{\mathrm{v}} \lambda^{2} \mathrm{P}_{\mathrm{a}}}{\mu_{\mathrm{g}} \kappa \mathrm{T}} \frac{\partial \mathrm{P}_{\mathrm{g}}}{\partial \mathrm{r}}
$$

The capillary pressure relates the gas pressure to the liquid pressure through the relationship:

$$
P_{c}(S, T)=P_{g}-P_{1}
$$

where $P_{c}$ is the capillary pressure, which depends on both saturation and temperature. The capillary pressure depends on the temperature through:

$$
\mathrm{P}_{c}(S, T)=\mathrm{P}_{c}\left(S, \mathrm{~T}_{0}\right)\left[1+\frac{\mathrm{d} \ln \sigma}{\mathrm{dT}}\left(\mathrm{T}-\mathrm{T}_{0}\right)\right]
$$

where $\sigma$ is the surface tension of water and $\mathrm{T}_{0}$ is a reference temperature.

The heat transport equation is given by: 


$$
[\rho c] \text { ave } \frac{\partial T}{\partial t}+\left(c_{1} j_{1}+c_{v} j_{v}+c_{a} j_{a}\right) \frac{\partial T}{\partial r}=\frac{1}{r^{i}} \frac{\partial}{\partial r}\left[r^{i} K \frac{\partial T}{\partial r}\right]-F_{v} L
$$

where

$$
[\rho c]_{\text {ave }}=(1-\phi) \rho_{r} c_{r}+\phi S \rho_{1} c_{1}
$$

$\rho_{r}$ is the rock grain density, $c_{r}$ is the rock grain specific heat,

$c_{1}$ is the liquid specific heat, $c_{y}$ is the vapor specific heat, $c_{a}$ is

the air specific heat, $K$ is the thermal conductivity of the mixture, and $L$ is

the latent heat of vaporization.

The characteristic curves (the relative permeability versus saturation, the capillary pressure versus saturation, the derivative of saturation with respect to capillary pressure), and the thermal conductivity versus saturation and temperature are supplied to PETROS through user-written function subprograms. Other parameters, such as the various diffusion coefficients, the water viscosity, the saturation vapor pressure of water, and the default values of the characteristic curves and thermal conductivity, are supplied internally in the code as function subprograms. Constants, such as gas viscosity, specific heats, and water density, can either be set at default values or supplied by the user. The user can also choose between equilibrium and nonequilibrium vapor pressure models.

The above equations are solved numerically by a finite-difference method. The equations are differenced in both space and time. Backward differencing in time results in fully implicit equations. Saturations can be spatially discontinuous between two different materials or soil/rock types. PETROS handles this discontinuity by using the average saturation from each side of the interface between the materials. The saturation and temperature equations are solved with a tridiagonal algorithm. Because the vapor and air partial pressure equations are strongly coupled, they are solved with a block tridiagonal algorithm. Dirichlet and Neumann boundary conditions can be applied to all the equations. 
CHAPTER 3

TEST PROBLEMS

In this section of the report, a description of the various test problems used in the evaluation of the the strongly heat-driven flow codes is presented. The codes were compared to five problems with an exact or numerical solution and three problems with an experimental result.

The problems ranged over various degrees of complexity. They were chosen to test various uncoupled aspects of the codes, such as two-phase flow or heat transport, before testing coupled processes. As such, the problems consist of an infiltration problem, heat transport problems in one and multiple dimensions, and coupled problems in one and multiple dimensions. It is believed that, if the codes cannot adequately simulate simple uncoupled processes, they cannot adequately simulate relatively complex coupled processes expected at an HLW repository.

None of the problems described below are considered to represent actual conditions at an HLW repository. At most, they represent some of the processes that can occur. Finding problems with exact solutions representive of expected HLW conditions is not possible. The same is true of problems with experimental results. Therefore, at most, the problems described below can only be expected to represent some simplification of the conditions at an HLW repository.

\subsection{Test Problems With Exact or Numerical Solutions}

\subsubsection{One-Dimensional Horizontal Infiltration}

The one-dimensional horizontal infiltration problem was originally solved by Phillip (1955) and is described in Ross et al. (1982). In this problem, a semi-infinite horizontal tube filled with a homogeneous soil is partially saturated with water. Air is not accounted for and is, in essence, a passive spectator. The tube of soil is held saturated at one end and, as time progresses, the moisture at the saturated end is pulled into the porous matrix. The purpose of this problem is to test the various codes' capabilities to simulate transient unsaturated flow in a porous medium. A schematic of the problem is presented in Figure 3.1.

The following data are applicable to the infiltration problem:

initial moisture content hydraulic conductivity porosity
0.20

$1.1574 \times 10^{-7} \mathrm{~m} / \mathrm{s}(1 \mathrm{~cm} /$ day $)$

0.45

The relative hydraulic conductivity characteristic curve of the unsaturated medium is described by the following curve (Figure 3.2): 


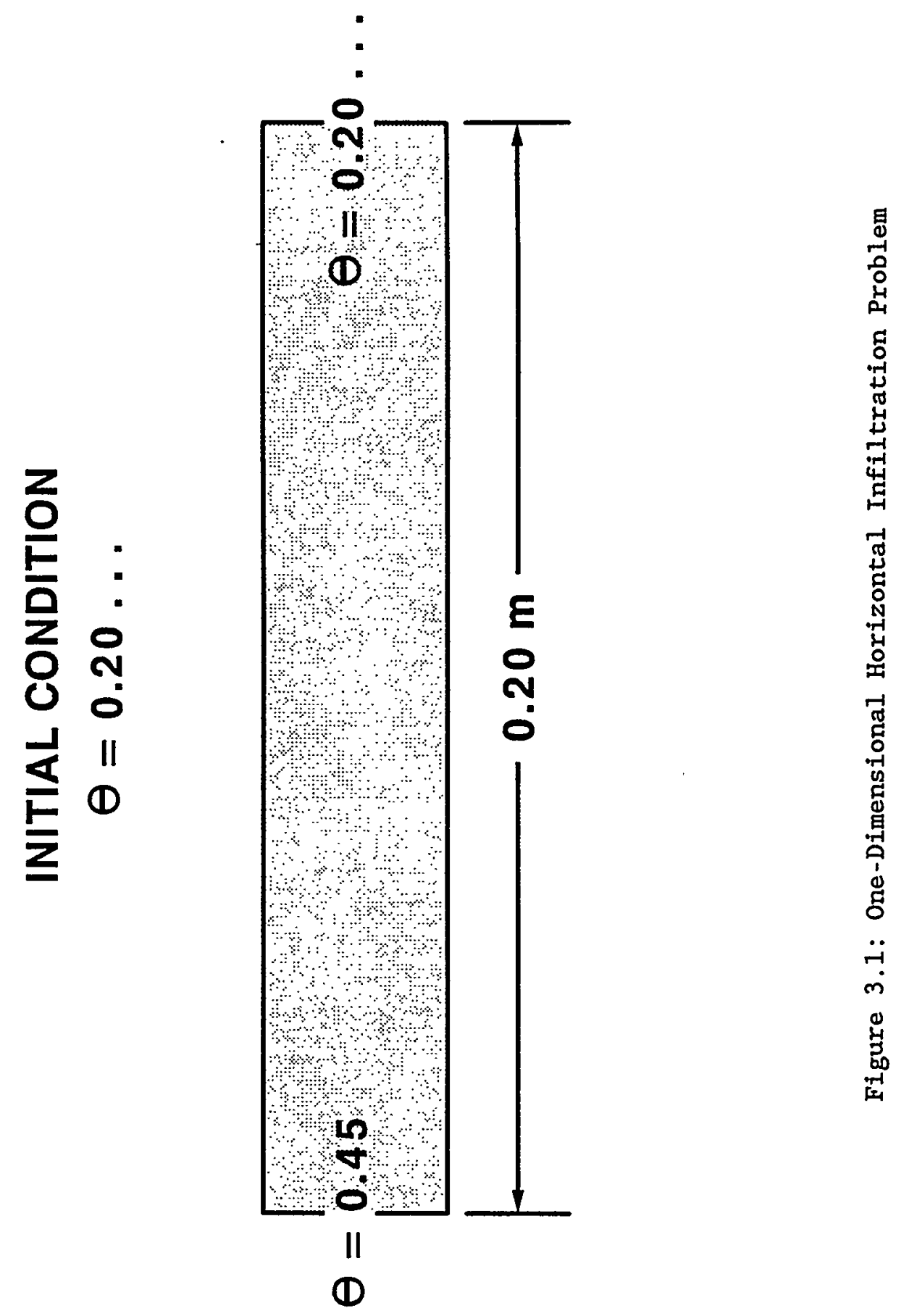




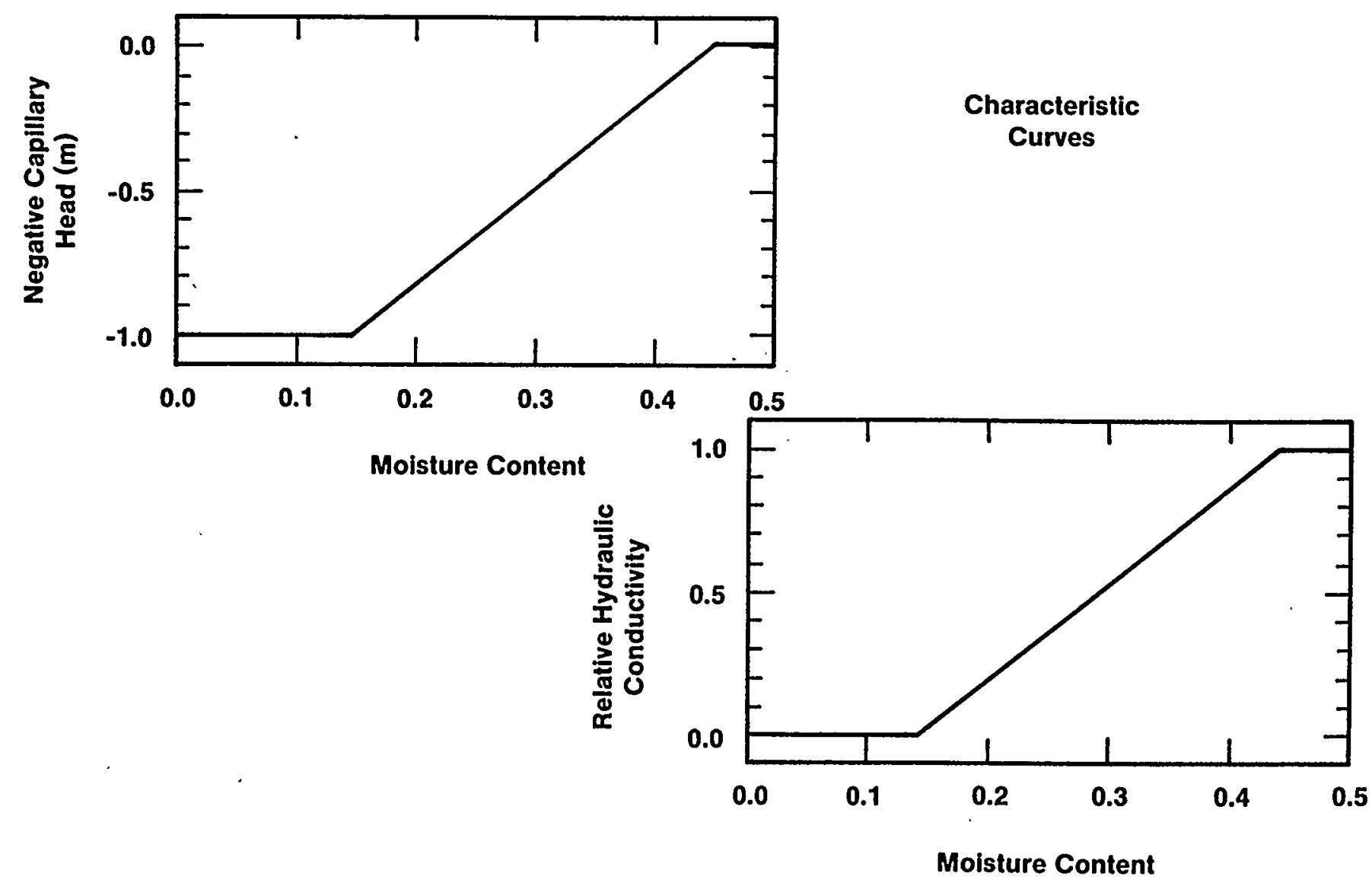

Figure 3.2: Characteristic Curves for the One-Dimensional Horizontal Infiltration Problem 


$$
k_{r}(\theta)=\left\{\begin{array}{cl}
{\left[\frac{\theta-\theta_{r}}{\theta_{s}-\theta_{r}}\right]} & \text { if } \theta \geq \theta_{r} \\
0 & \text { if } \theta<\theta_{r}
\end{array}\right.
$$

where $\mathrm{k}_{\mathrm{r}}$ is the relative hydraulic conductivity, $\theta$ is the moisture content, $\theta_{s}$ is the saturated moisture content (or porosity), and $\theta_{r}$ is the residual moisture content. The residual moisture content specified for the problem is 0.15 . The capillary head characteristic curve is given by (Figure 3.2):

$$
\psi_{r}(\theta)=\left\{\begin{array}{cl}
\psi_{r}\left[\frac{\theta-\theta_{r}}{\theta_{s}-\theta_{r}}\right] & \text { if } \theta>\theta_{r} \\
\psi_{r} & \text { if } \theta \leq \theta_{r}
\end{array}\right.
$$

where $\psi_{r}$ is a constant and is the limit of capillary head as the soil dries. Its value for this problem is $1.00 \mathrm{~m}$.

Ross et al. (1982) specify the exact solution at times of $864 \mathrm{~s}$ (0.01 days), $5184 \mathrm{~s}$ (0.06 days), and $9504 \mathrm{~s}$ (0.11 days). It is necessary to compare code predictions at these same simulation times. These results of the exact solution indicate that the semi-infinite soil tube can be represented by a finite one with a minimum length of $0.2 \mathrm{~m}$.

\subsubsection{One-Dimensional Heat Transport}

Avdonin (1964) originally solved the one-dimensional heat transport problem, which is described in Ross et al. (1982). In this problem cold water is injected into an semi-infinite, $100-\mathrm{m}$-thick hot water aquifer, which is overlain and underlain by an overburden and underburden, respectively. For modeling purposes, the overburden and underburden are neglected in order to simplify the problem and adiabatic boundary conditions are prescribed there. This, in effect, prevents heat from escaping the aquifer and reduces the equation governing heat transport to the convective-diffusive equation. Figure 3.3 presents a diagram of the problem. This problem was used to test the ability of the various codes to simulate convective-diffusive heat transport.

The convective-diffusive equation for heat transport describes the temperature field in the aquifer:

$$
\rho_{\mathrm{m}} c_{\mathrm{m}} \frac{\partial \mathrm{T}}{\partial t}+u_{\mathrm{w}} \rho_{\mathrm{w}} c_{\mathrm{w}} \frac{\partial \mathrm{T}}{\partial \mathrm{x}}=\mathrm{K}_{\mathrm{m}} \frac{\partial^{2} \mathrm{~T}}{\partial \mathrm{x}^{2}}
$$




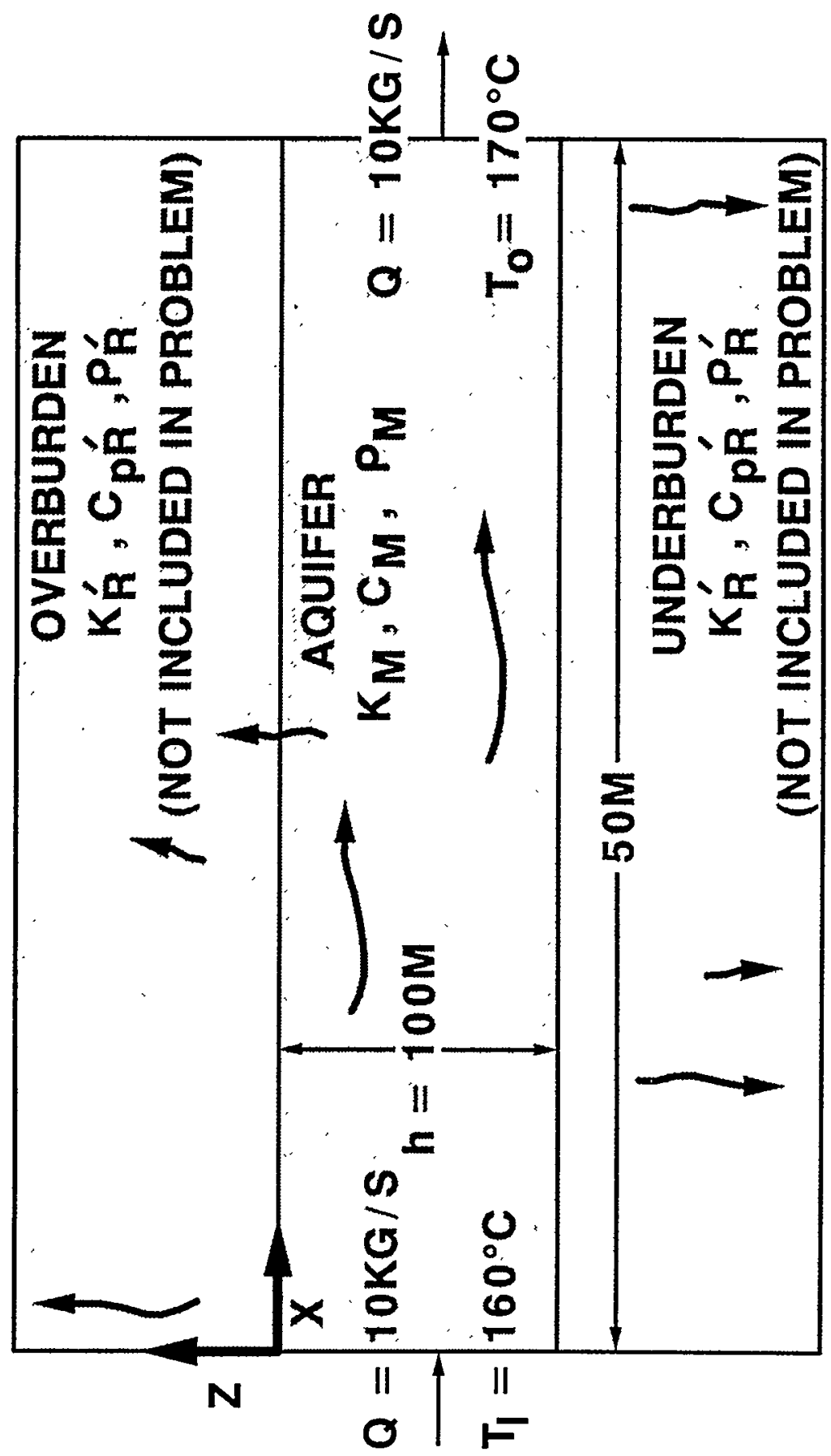

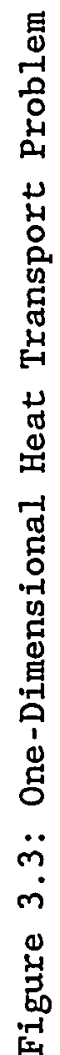


where $\rho_{\mathrm{m}}$ is the density of the saturated porous medium, $\mathrm{c}_{\mathrm{m}}$ is the specific heat of the saturated porous medium, $u_{w}$ is the Darcy velocity of the water, $\rho_{w}$ is the density of water, $c_{w}$ is the specific heat of the water, $K_{m}$ is the thermal conductivity of the saturated porous medium, $T$ is temperature, $\mathrm{x}$ is distance, and $t$ is time.' At $\mathrm{x}=0$, the temperature is held fixed at $160^{\circ} \mathrm{C}$. The temperature at the far end is fixed at the same temperature as the aquifer, $170^{\circ} \mathrm{C}$. Ross et al. (1982) present a corrected solution of the Avdonin (1964) solution to the convective-diffusive equation. The Ross et al. solution includes the effects of the overburden and underburden. However, if the overburden and underburden components are eliminated from the solution, it reduces to the solution of the convective-diffusive equation with a Dirichlet boundary condition:

$$
\frac{T(x, t)-T(x, 0)}{T(0, t)-T(x, 0)}=\frac{1}{2}\left\{\operatorname{erfc}\left[\frac{x-v t}{\sqrt{4 D t}}\right]+\exp \left[\frac{v x}{D}\right] \operatorname{erfc}\left[\frac{x+v t}{\sqrt{4 D t}}\right]\right\}
$$

where $\mathrm{v}=\mathrm{u}_{\mathrm{w}} \rho_{\mathrm{w}} \mathrm{c}_{\mathrm{w}} /\left(\rho_{\mathrm{m}} \mathrm{c}_{\mathrm{m}}\right), \mathrm{D}=\mathrm{K}_{\mathrm{m}} /\left(\rho_{\mathrm{m}} \mathrm{c}_{\mathrm{m}}\right), \mathrm{T}(0, \mathrm{t})=$

temperature at $x=0$, and $T(x, 0)$ is the initial temperature of the aquifer.

Additional parameters must be specified to complete the problem. These include a fully saturated aquifer specific heat of $1000 \mathrm{~J} / \mathrm{kg}-{ }^{\circ} \mathrm{C}$, a fully saturated aquifer density of $2500 \mathrm{~kg} / \mathrm{m}^{3}$, a water specific heat of 4185 $\mathrm{J} / \mathrm{kg}-{ }^{\circ} \mathrm{C}$, a water density of $919 \mathrm{~kg} / \mathrm{m}^{3}$, a water mass flow rate of 10 $\mathrm{kg} / \mathrm{s}$, an aquifer porosity of 0.2 , a fully saturated aquifer thermal conductivity of $20 \mathrm{~W} / \mathrm{m}-{ }^{\circ} \mathrm{C}$, and an aquifer thickness of $100 \mathrm{~m}$. Other water properties are taken from the steam tables (Meyer et al., 1968). Buoyancy of heated water within the aquifer is neglected. Code predictions are required at $130000 \mathrm{~s}$ after the start of cold water injection. Previous modeling studies indicate that a $40-\mathrm{m}$-long aquifer is enough to approximate the semi-infinite aquifer (Ward et al., 1984).

\subsubsection{Radial Heat Transport}

The radial heat transport problem is very similar to the linear heat transport problem described in Section 3.1.2. The problem was originally solved by Avdonin (1964) and is described by Ross et al. (1982). The only difference between the radial heat transport problem and the linear one is that the former problem is formulated in a radial coordinate system while the linear problem is formulated in a one-dimensional Cartesian system. In both cases, the hydraulic and thermal properties are the same, and the overburden and underburden are neglected. Figure 3.4 presents a diagram of the problem. This problem was used to test the codes' ability to solve heat transport problems in multidimensional coordinate systems.

The heat transport is governed by the radial convective-diffusion equation:

$$
\rho_{\mathrm{m}} c_{\mathrm{m}} \frac{\partial \mathrm{T}}{\partial \mathrm{t}}+u_{\mathrm{w}} \rho_{\mathrm{w}} c_{\mathrm{w}} \frac{\partial \mathrm{T}}{\partial \mathrm{r}}=\mathrm{K}_{\mathrm{m}_{\mathrm{r}}} \frac{1}{\partial \mathrm{r}}\left[\mathrm{r} \frac{\partial \mathrm{T}}{\partial \mathrm{r}}\right]
$$




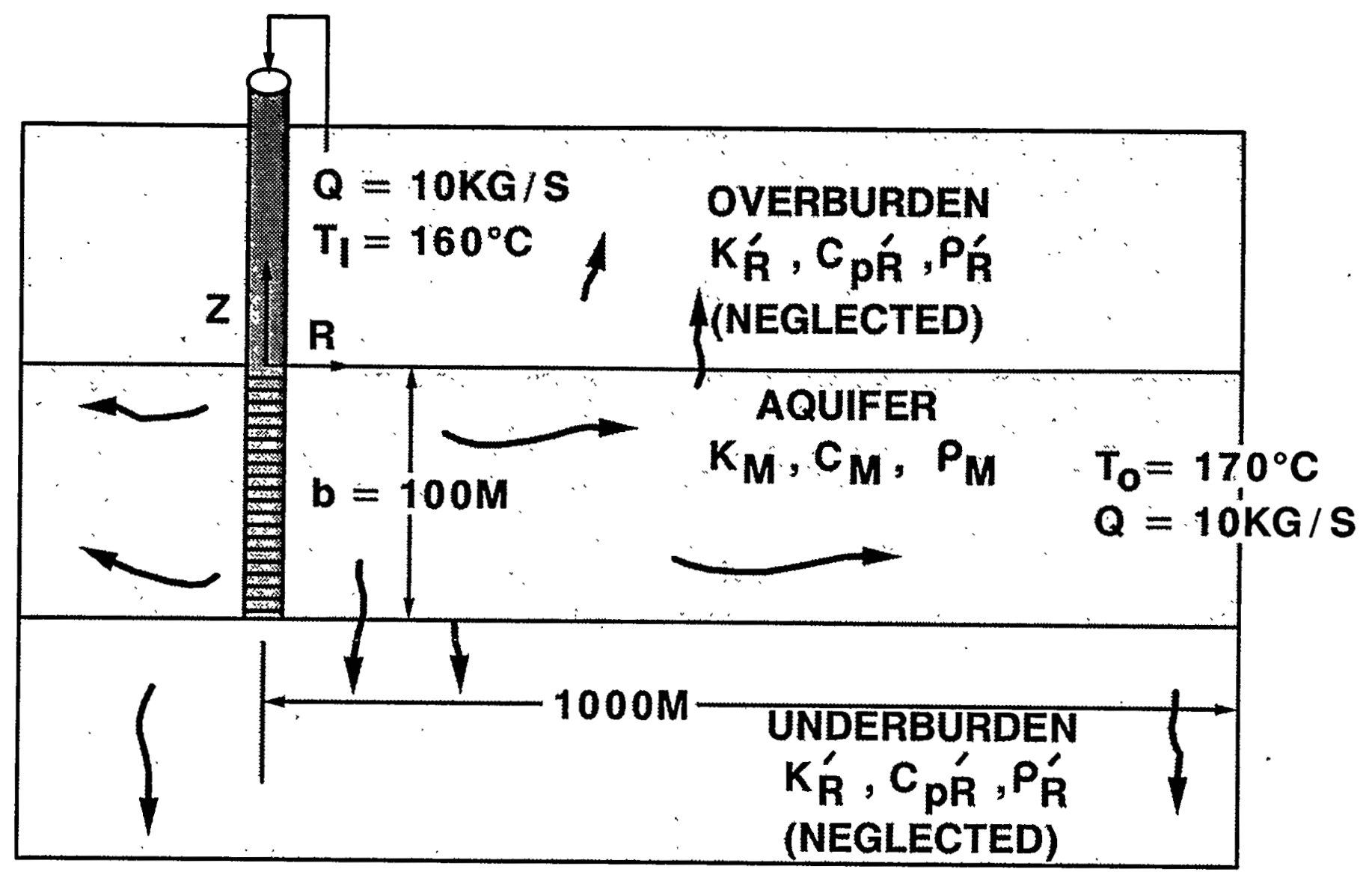

Figure 3.4: Radial Heat Transport Problem 
where $r$ is radial distance and the other parameters are the same as those defined in Section 3.1.2. Since this is a radial coordinate problem, the water Darcy velocity, $u_{w}$, depends on the radial distance. This means that the Darcy velocity is defined by:

$$
u_{w}=\frac{Q}{2 \pi r b}
$$

where $Q$ is the volumetric injection rate of cold water and $b$ is the aquifer thickness. At an infinitesimally small injection well, the temperature is fixed at $160^{\circ} \mathrm{C}$. At a distance of $1000 \mathrm{~m}$ from the injection well the temperature is fixed at $170^{\circ} \mathrm{C}$, the initial temperature of the aquifer.

Avdonin (1964) provides a solution to the above problem which includes the effects of the overburden and underburden. However, if the overburden and underburden are neglected and the temperature does not reach the outer boundary, the solution reduces to:

$$
\frac{T(r, t)-T(r, 0)}{T(0, t)-T(r, 0)}=\frac{\Gamma\left(\nu, \omega^{2} / 4 \tau\right)}{\Gamma(\nu)}
$$

where $\Gamma(\nu)$ is the gamma function, $\Gamma\left(\nu, \omega^{2} / 4 \tau\right)$ is the complementary incomplete gamma function, $\nu=Q \rho_{\mathrm{w}} \mathrm{c}_{\mathrm{w}} /\left(4 \pi \mathrm{bK} \mathrm{K}_{\mathrm{m}}\right), \omega=2 \mathrm{r} / \mathrm{b}$, and $\tau=4 \mathrm{~K}_{\mathrm{m}} \mathrm{t} /\left(\rho_{\mathrm{m}} \mathrm{c}_{\mathrm{m}} \mathrm{b}^{2}\right)$. This problem requires code output of temperature as a function of distance at $10^{9} \mathrm{~s}$.

\subsubsection{Radial Boiling Front}

The radial boiling front problem is described and solved by Garg (1980). In this problem water and/or steam is pumped from the center of an infinitely large, hot water aquifer. It is possible for water within a portion of the aquifer to vaporize due to lowering of the aquifer pressure caused by the pumping of hot water. Therefore, this is a one-component, two-phase flow and heat transport problem. Air is not included and capillary pressure is neglected in this problem. A diagram of the problem, which indicates four possible cases, is presented in Figure 3.5. Only case D is considered in this report. In this case a 100-m-thick aquifer is initially saturated with water at a temperature of $300^{\circ} \mathrm{C}$. Water is removed at a rate of $0.14 \mathrm{~kg} / \mathrm{s}$ such that water in the aquifer vaporizes. This vaporized water, forming a flash front, advances into the aquifer with time. The approximate equation governing flow in the two-phase region is:

$$
\frac{\partial \mathrm{P}_{2}}{\partial \mathrm{t}}=\frac{(\mathrm{k} / \nu)_{2}}{\left(\phi \rho \mathrm{c}_{\mathrm{t}}\right)_{2}} \frac{1}{\mathrm{r}} \frac{\partial}{\partial \mathrm{r}}\left[\mathrm{r} \frac{\partial \mathrm{P}_{2}}{\partial \mathrm{r}}\right]
$$

where $P_{2}$ is pressure in the two-phase region, $t$ is time, $r$ is radial distance, $(\mathrm{k} / \nu)_{2}$ is total kinematic mobility in the two-phase region, 


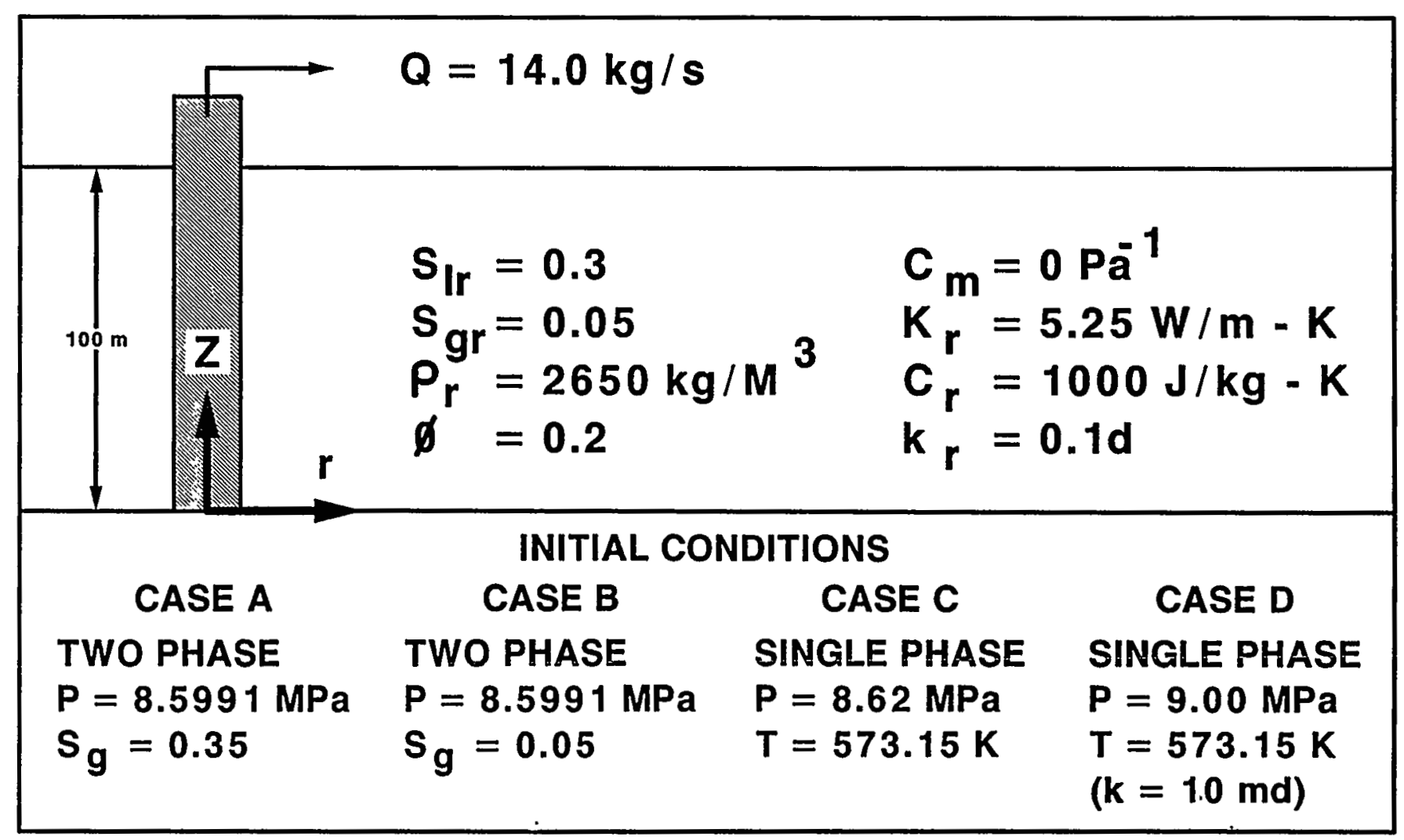

Figure 3.5: Radial Boiling Front Problem 
$\phi$ is porosity, $\rho$ is mixture (liquid and gas) density, and $c_{t}$ is total compressibility. The kinematic mobility is a combination of the liquid and gas mobilities:

$$
(\mathrm{k} / \nu)_{2}=\mathrm{kk}_{\mathrm{rg}} \rho_{\mathrm{g}} / \mu_{\mathrm{g}}+\mathrm{kk}_{\mathrm{rl}} \rho_{1} / \mu_{1}
$$

where $\mathrm{k}$ is the porous medium intrinsic permeability, $\mathrm{k}_{\mathrm{rg}}$ is the gas relative permeability, $k_{r 1}$ is the liquid relative permeability, $\rho_{g}$ is the gas density, $\rho_{1}$ is the liquid density, $\mu_{\mathrm{g}}$ is the gas dynamic viscosity, and $\mu_{1}$ is the liquid dynamic viscosity. The mixture density in the two-phase region is:

$$
\rho=s_{g} \rho_{g}+s_{1} \rho_{1}
$$

where $S_{g}$ is gas saturation and $S_{1}$ is liquid saturation. The total compressibility depends on the rock compressibility and the liquid/gas mixture compressibility, the latter of which depends on both pressure and temperature.

The governing flow equation in the one-phase region is:

$$
\frac{\partial \mathrm{P}_{1}}{\partial t}=\frac{(\mathrm{k} / \nu)_{1}}{\left(\phi \rho \mathrm{c}_{t}\right)_{1}} \frac{1}{\mathrm{r}} \frac{\partial}{\partial \mathrm{r}}\left[\mathrm{r} \frac{\partial \mathrm{P}_{1}}{\partial \mathrm{r}}\right]
$$

where $(\mathrm{k} / \nu)_{1}$ is the total mobility in the single-phase region, which is defined as:

$$
(\mathrm{k} / \nu)_{1}=\mathrm{kk}_{\mathrm{r1}} \rho_{1} / \mu_{1}
$$

The total compressibility in the one-phase region is defined as:

$$
c_{t}=c_{m}(1-\phi) / \phi+c_{f}
$$

where $c_{m}$ is the rock compressibility and $c_{f}$ is the liquid compressibility. The density in the one-phase region is simply the liquid density, $\rho_{1}$. The boundary condition for fluid removal at the well is:

$$
\lim _{r \rightarrow 0}\left[\frac{\partial P_{j}}{\partial r}\right]=-\frac{M}{2 \pi b(k / \nu)_{j}}
$$

where $M$ is the mass withdrawal rate, $b$ is the aquifer thickness, and $j$ is either "1" or "2" depending on whether the region near the wellbore is either 
a one- or a two-phase region. The total kinematic mobility has been defined previously. The boundary condition at the outer edge of the aquifer is:

$$
\lim _{\mathrm{I} \rightarrow \infty} \mathrm{P}_{1}=\mathrm{P}_{\mathbf{i}}
$$

where $P_{i}$ is the initial pressure of the aquifer. In order to completely describe the mathematics, two compatibility conditions are required at the flash front, $r=R$. First, a continuous flux condition,

$$
\left.(k / \nu) 2 \frac{\partial \mathrm{P}_{2}}{\partial \mathrm{r}}\right|_{\mathrm{r}=\mathrm{R}}=\left.(\mathrm{k} / \nu)_{1} \frac{\partial \mathrm{P}_{1}}{\partial \mathrm{r}}\right|_{\mathrm{r}=\mathrm{R}}
$$

where $R$ is the distance where the flash front occurs as a function of time, is necessary. The second compatibility condition prescribes a continuous pressure:

$$
\left.\mathrm{P}_{2}\right|_{\mathrm{r}=\mathrm{R}}=\left.\mathrm{P}_{1}\right|_{\mathrm{r}=\mathrm{R}}
$$

Both pressures are equal to the saturation pressure, $P_{S}(T)$, where $T$ is temperature. Garg (1980) provides a solution to the problem. However, for purposes of this report, only long-time asymptotic solutions of pressure in the wellbore are of interest. The long-time asymptotic wellbore pressure distribution is given as:

$$
P_{w}(t)=P_{s}-\frac{M E i\left(-\lambda^{2}\right)}{4 \pi b(k / \nu)_{2}}-\frac{1.15 M}{2 \pi b(k / \nu)_{2}}\left[\log _{10}\left[\frac{(k / \nu)_{2} t}{r_{w}{ }^{2}\left(\phi \rho c_{t}\right)_{2}}\right]+0.351\right)
$$

where $E i\left(-\lambda^{2}\right)$ is the negative exponential integral with argument, $-\lambda^{2}, P_{w}$ is the wellbore pressure, and $r_{w}$ is the wellbore radius. The important thing to realize about the above solution is that it represents a linear relationship between the wellbore pressure and $\log (t)$.

The parameter, $-\lambda^{2}$, can be estimated from the nonlinear equation:

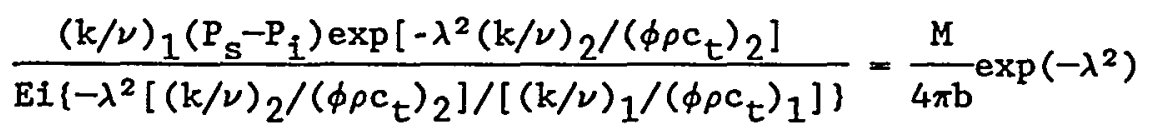

Garg (1980) gives a numerical solution to this problem. The numer-ical solution exhibits the same qualitative behavior as the long-time asymptotic solution. Therefore, it was decided that the numerical solution generated by Garg was adequate for the code comparisons. 
To simulate the radial boiling front problem, Garg (1980) used the following hydrologic and thermal properties:

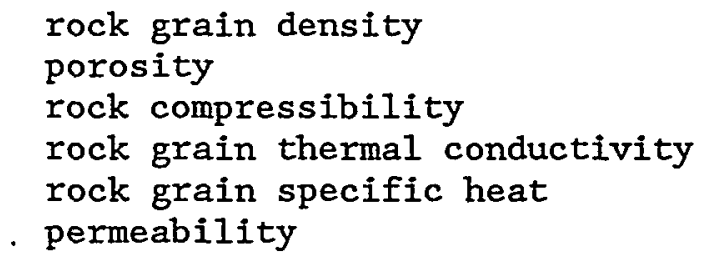

$$
\begin{aligned}
& 2650 \mathrm{~kg} / \mathrm{m}^{3} \\
& 0.2 \\
& 0 \mathrm{MPa}^{-1} \\
& 5.25 \mathrm{~W} / \mathrm{m}-{ }^{\circ} \mathrm{C} \\
& 1000 \mathrm{~J} / \mathrm{kg}^{\circ} \mathrm{C} \\
& 9.8697 \times 10^{-15} \mathrm{~m}^{2}
\end{aligned}
$$

The relative permeabilities were represented with Corey's equations:

$$
\begin{aligned}
& s^{*}=\left[\frac{s_{1}-s_{1 r}}{1-s_{1 r}-s_{g r}}\right]^{4} \\
& k_{r 1}=\left(s^{*}\right)^{4} \\
& k_{r g}=\left(1-s^{*}\right)^{2}\left(1-s^{* 2}\right)
\end{aligned}
$$

where $\mathrm{S}_{1 \mathrm{r}}$ is the residual liquid saturation and $\mathrm{s}_{\mathrm{gr}}$ is the residual gas saturation. $S_{1 r}$ and $S_{g r}$ have values of 0.30 and 0.05 , respectively.

\subsubsection{Heat Pipe}

The heat pipe problem is described and modeled by Udell and Fitch (1985). Figure 3.6 presents a schematic of the problem. In this problem, a one-dimensional horizontal layer of porous medium filled with water and a two-component gas (nitrogen and water vapor) is subject to a heat flux at the right end. The result is that moisture collects at the left end of the porous medium layer while the right end, which is subject to the heat flux, dries. In addition, near the saturated left end the temperature increases strongly before approaching a constant value at the heat flux end. Finally, the mass fraction of nitrogen in the two-component gas decreases rapidly to zero near the left end of the porous medium layer. This problem was used to test the codes' capability to simulate coupled two-phase flow, heat transport, and evaporation processes.

Udell and Fitch (1985) formulated the steady-state flow and heat transport equations into a set of four one-dimensional equations. These equations, for a horizontal porous medium layer, are:

$$
\frac{\partial \mathrm{x}}{\partial \mathrm{S}}=\frac{-\frac{\partial \mathrm{P}_{\mathrm{C}}}{\partial \mathrm{S}}\left[\frac{1}{\rho_{1} \mathrm{~g}}\right]}{\lambda \omega \frac{1}{\mathrm{k}_{\mathrm{rg}}}\left[\frac{1}{1-\mathrm{y}}+\frac{\nu_{1}}{\nu_{\mathrm{g}}} \frac{\mathrm{k}_{\mathrm{rg}}}{\mathrm{k}_{\mathrm{r} I}}\right]}
$$




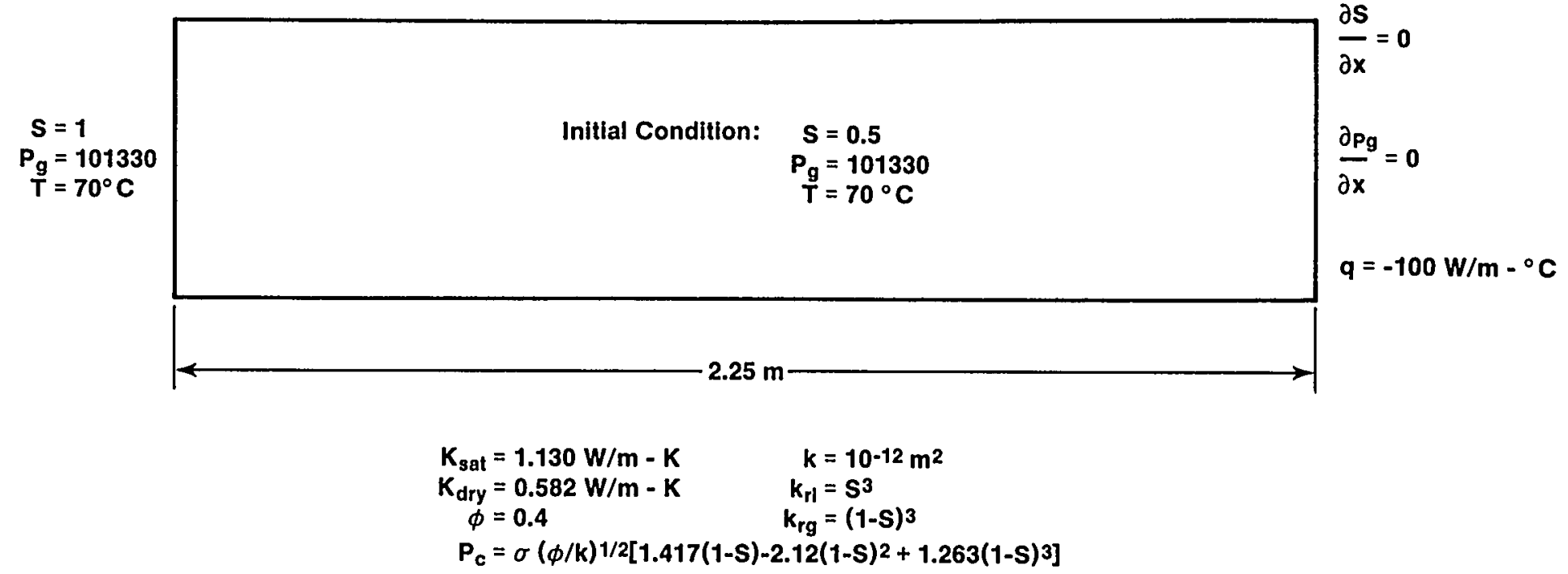

Figure 3.6: Heat Pipe Problem 


$$
\begin{aligned}
& \frac{\partial \mathrm{P}_{\mathrm{g}}}{\partial \mathrm{S}}=\frac{\frac{\partial \mathrm{P}_{\mathrm{c}}}{\partial \mathrm{S}}}{1+\left[\frac{\nu_{1}}{\nu_{\mathrm{g}}} \frac{\mathrm{k}_{\mathrm{rg}}}{\mathrm{k}_{\mathrm{r} 1}}\right][1-\mathrm{y}]} \\
& \frac{\partial y}{\partial S}=\frac{-\frac{\partial P_{c}}{\partial S}\left[\frac{k}{\nu_{g} \rho_{g} D_{e f f}}\right]}{\frac{1}{k_{r g}}\left[\frac{1}{1-y}+\frac{\nu_{1}}{\nu_{g}} \frac{k_{r g}}{k_{r l}}\right]} \\
& \frac{\partial T}{\partial S}=\frac{\frac{\partial P_{c}}{\partial S}\left[\frac{1-\lambda}{\lambda} \frac{h_{f g}}{\nu_{g}}\right]}{\frac{1}{k_{r g}}\left[\frac{1}{1-y}+\frac{\nu_{1}}{\nu_{g}} \frac{k_{r g}}{k_{r l}}\right]}
\end{aligned}
$$

where $\mathrm{x}$ is distance, $\mathrm{S}$ is normalized saturation, $\mathrm{P}_{c}$ is capillary pressure, $P_{g}$ is gas pressure, $\rho_{I}$ is liquid density, $\rho_{g}$ is gas density, $k$ is permeability, $k_{r g}$ is gas relative permeability, $k_{r l}$ is liquid relative permeability, $\nu_{g}$ is gas kinematic viscosity, $\nu_{1}$ is lîquid

kinematic viscosity, $y$ is molar fraction of noncondensable gas in the gas, $t$ is temperature, $g$ is gravity, and $h_{f g}$ is latent heat of vaporization. The ratio of convective heat flux to total heat flux, $\lambda$, is described by:

$$
\lambda=1+\frac{K \partial T / \partial x}{q}
$$

where $\mathrm{q}$ is the heat flux (positive to the right) and $\mathrm{K}$ is the thermal conductivity, which is described by:

$$
\mathrm{k}=\mathrm{k}_{\mathrm{dry}}+\sqrt{\mathrm{s}_{1}}\left(\mathrm{~K}_{\mathrm{wet}}-\mathrm{K}_{\mathrm{dry}}\right)
$$

where $K_{\text {wet }}$ is the thermal conductivity of the saturated porous medium, $\mathrm{K}_{\mathrm{dry}}$ is the thermal conductivity of the dry porous medium, and $\mathrm{s}_{1}$ is the liquid saturation. The constant, $\omega$, is defined by:

$$
\omega=\frac{q \nu_{g}}{h_{f g} k \rho_{1} g}
$$


The normalized saturation is simply:

$$
s=\frac{s_{1}-s_{1 r}}{1-s_{1 r}}
$$

where and $s_{1 r}$ is the residual saturation. The effective molecular diffusivity is given by:

$$
D_{\text {eff }}=\frac{\phi\left(1-S_{1}\right) D_{n c}}{r}
$$

where $\phi$ is porosity, $\tau$ is tortuosity, and $D_{n c}$ is the molecular diffusion coefficient of gas ' $n$ ' in gas mixture ' $n-c$ '. The term, $\left(1-S_{1}\right)$, accounts for blockage due to pore water. The characteristic curves for capillary pressure and relative permeability are described as:

$$
\begin{aligned}
& \mathrm{P}_{c}=\sigma(\phi / \mathrm{k})^{1 / 2}\left[1.417(1-\mathrm{S})-2.12(1-\mathrm{S})^{2} 2+1.263(1-\mathrm{S})^{3}\right] \\
& \mathrm{k}_{\mathrm{rg}}=(1-\mathrm{S})^{3} \\
& \mathrm{k}_{\mathrm{r} 1}=\mathrm{S}^{3}
\end{aligned}
$$

where $\sigma$ is the surface tension of water. The first-order differential equations described above are subject to the following boundary conditions:

$$
\begin{array}{ll}
S=1 & \text { at } x=0 \\
P_{g}=P_{g i} & \text { at } x=0 \\
T=T_{i} & \text { at } x=0 .
\end{array}
$$

In addition, $y$ must satisfy the boundary condition:

$$
T=\frac{T_{o}\left[1+\frac{P_{c}-y P_{g}}{h_{f g} \rho_{1}}\right]}{1-T_{o_{h_{f g}}} \frac{R}{h_{g}}\left[\frac{P_{g}(1-y)}{P_{o}}\right]}
$$

at $x=0$, where $T_{0}$ is a reference temperature, $P_{0}$ is a reference pressure, and $R$ is the condensable gas constant. The differential equations 
are formulated with respect to $S$ to improve the stability of the numerical solution.

Udell and Fitch (1985) solved the above set of differential equations for the properties listed in Table 3.1. Unless otherwise indicated, the properties are independent of temperature and pressure and are, thus, constants. The boundary conditions at $\mathrm{x}=0$ are:

$$
\begin{aligned}
& \mathrm{T}_{\mathrm{i}}=343.15 \mathrm{~K} \\
& \mathrm{P}_{\mathrm{gi}}=101330 \mathrm{~Pa} \\
& \mathrm{~S}=1 \\
& \mathrm{y}=0.6818 \text { (calculated from boundary equation). }
\end{aligned}
$$

The solution of Udell and Fitch (1985) is shown in Figure 3.7. It is noted that all the dependent variables reach asymptotic values within $2 \mathrm{~m}$ of the left boundary. This implies that when modeling this problem the right boundary of the modeled region should be $2 \mathrm{~m}$ or more from the left boundary. The right boundary conditions should reflect zero flux of both liquid and gas and a $100 \mathrm{~W} / \mathrm{m}^{2}$ heat flux directed inward. Boundary conditions for the left side are listed above.

In order to compare code predictions to the solution for this problem, the simulation needs to be carried out to steady-state conditions.

\subsection{Test Problems With Experimental Results}

\subsubsection{Two-Dimensional Infiltration}

The two-dimensional infiltration problem is described and solved numerically by Vauclin et al. (1979). This problem was used to test the codes' ability to model unsaturated flow under laboratory conditions. Figure 3.8 presents only the right half of the problem. In this problem, a 2-m-high by $6-m-1$ ong vertical slab of soil is recharged at a rate of $4.111 \times 10^{-5} \mathrm{~m} / \mathrm{s}(14.8$ $\mathrm{cm} / \mathrm{hr}$ ) over a length of $1 \mathrm{~m}$. The recharge zone is located at the center of the top of the slab.

The slab is bounded on each side by lateral trenches, each filled with $0.65 \mathrm{~m}$ of water. Both the bottom and line of symmetry of the slab are considered impermeable to flow. Prior to the initiation of flow, the bottom of the slab is filled with water to a level equal to the water level in the trench. In the unsaturated zone, there is a no flow initial condition.

The equation governing flow in both the unsaturated and saturated zones is:

$$
C(h) \frac{\partial H}{\partial t}=\frac{\partial}{\partial x}\left[K(h) \frac{\partial H}{\partial x}\right]+\frac{\partial}{\partial z}\left[K(h) \frac{\partial H}{\partial z}\right]
$$


Table 3.1: Representative Fluid and Medium Properties for the Heat Pipe Problem

$\begin{array}{ll}\text { dry rock thermal conductivity } & 0.582 \mathrm{~W} / \mathrm{m}-\mathrm{K} \\ \text { wet rock thermal conductivity } & 1.130 \mathrm{~W} / \mathrm{m}-\mathrm{K} \\ \text { gas density } & 0.960 \mathrm{~kg} / \mathrm{m}^{3} \\ \text { liquid density } & 958.400 \mathrm{~kg} / \mathrm{m}^{3} \\ \text { gas kinematic viscosity } & 21.0 \times 10^{-6} \mathrm{~m}^{2} / \mathrm{s} \\ \text { liquid kinematic viscosity } & 0.3066 \times 10^{-6} \mathrm{~m}^{2} / \mathrm{s} \\ \text { heat of vaporization } & 2.257 \times 10^{6} \mathrm{~J} / \mathrm{kg} \\ \text { permeability } & 1.0 \times 10^{-12} \mathrm{~m}^{2} \\ \text { surface tension } & 0.05878 \mathrm{~N} / \mathrm{m} \\ \text { molecular diffusion of gas } & 26.0 \times 10^{-6} \mathrm{~m}^{2} / \mathrm{s} \\ \text { condensable gas constant } & 461.52 \mathrm{~J} / \mathrm{kg}^{-\mathrm{mole}-\mathrm{K}} \\ \text { reference temperature } & 373.15 \circ \mathrm{K} \\ \text { reference pressure } & 101330.0 \mathrm{~Pa} \\ \text { gravitational constant } & 9.817 \mathrm{~m} / \mathrm{s}^{2} \\ \text { porosity } & 0.4 \\ \text { tortuosity } & 2.0 \\ \text { heat flux } & 100.0 \mathrm{~W} / \mathrm{m}^{2}\end{array}$


Ratio of Convective to Total Heat Flux $(\lambda)$

Normalized Saturation ( $\mathbf{S}$ )

Molar Fraction of Non-Condensable Gas $(Y)$

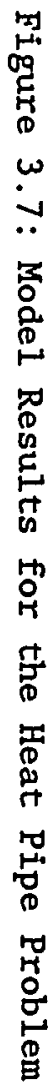

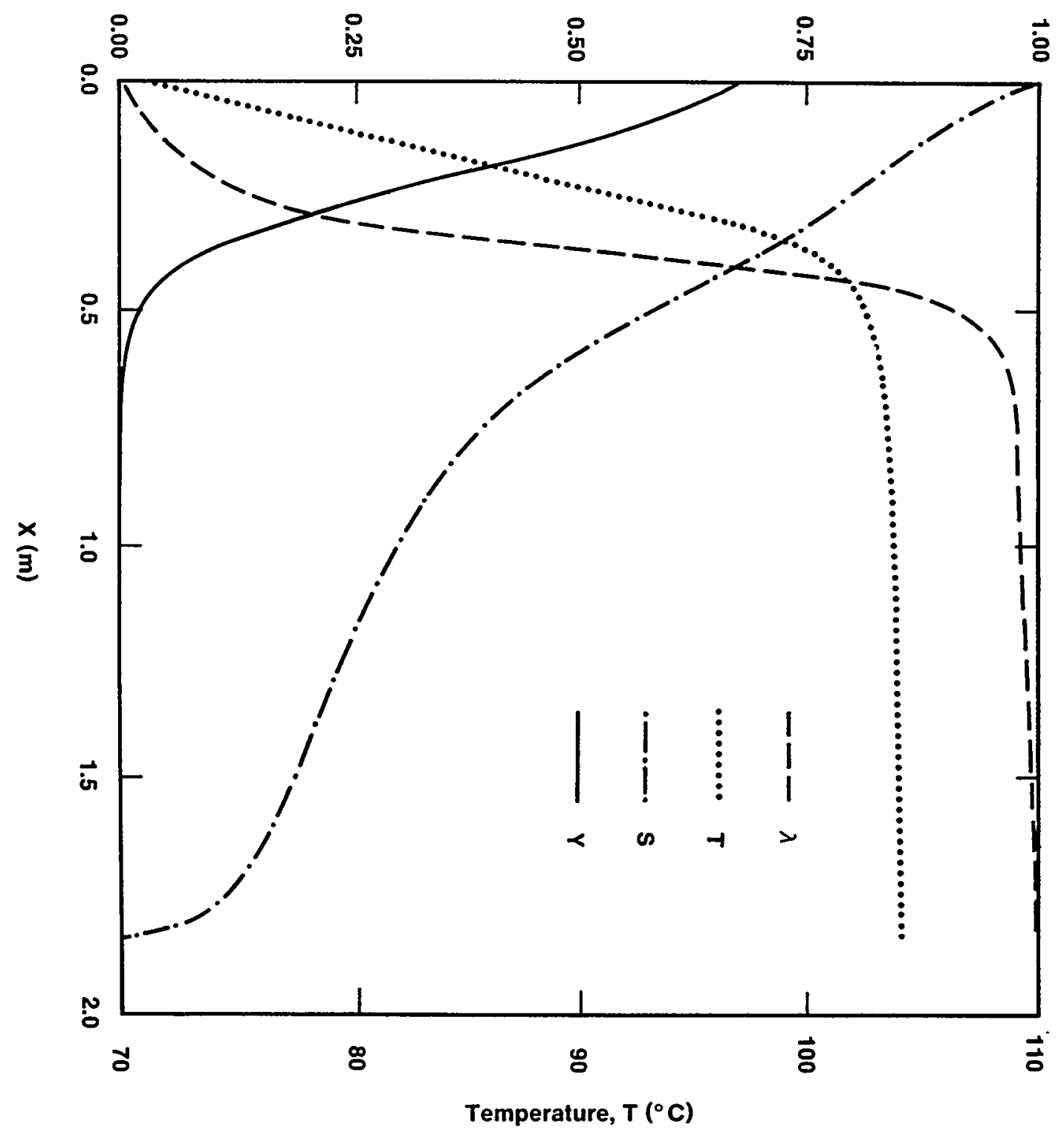




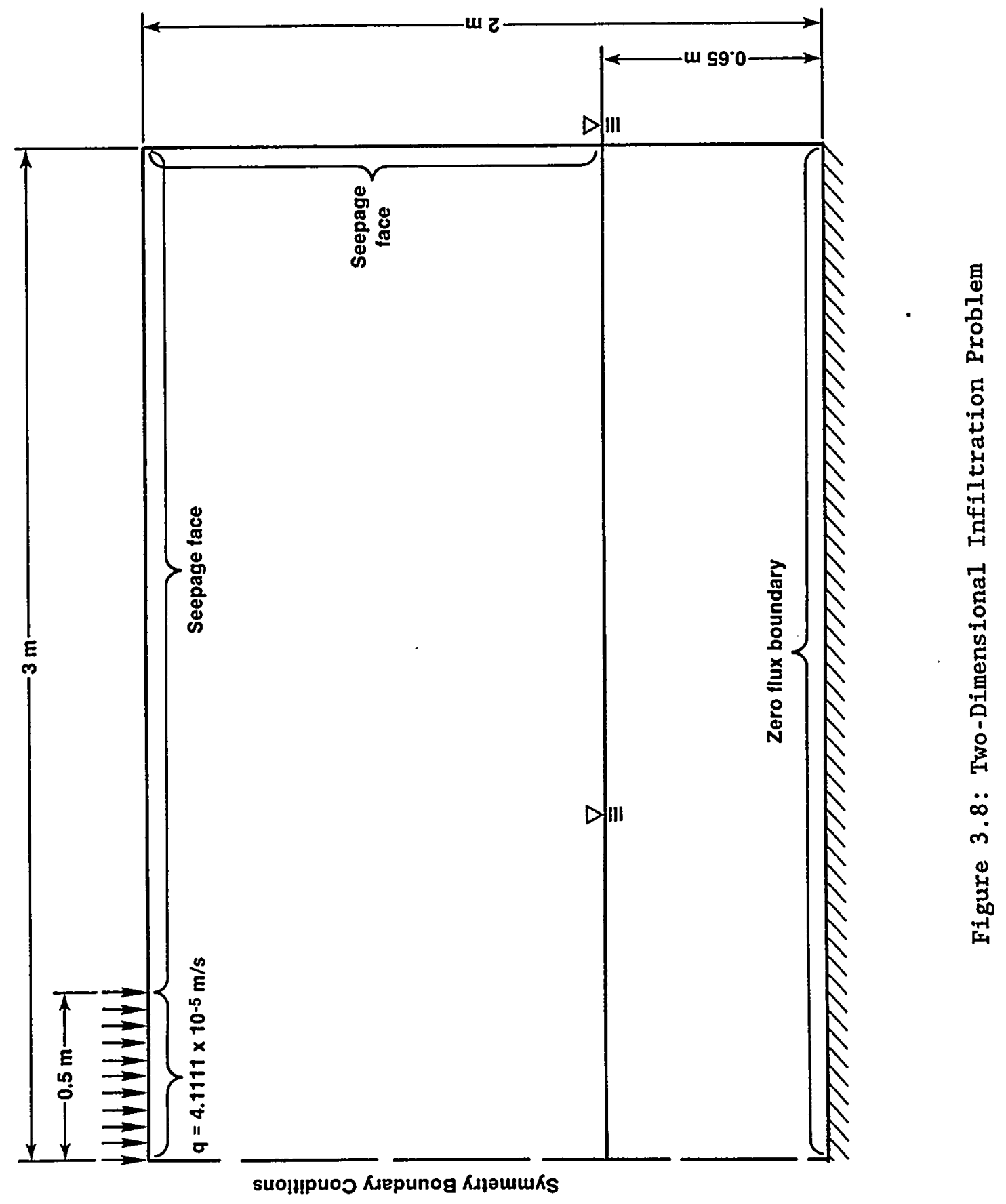

$-33-$ 
where $\mathrm{H}$ is the total hydraulic head, $\mathrm{h}$ is the capillary head, $\mathrm{C}(\mathrm{h})$ is the moisture capacity, defined as $\mathrm{d} \theta / \mathrm{dh}$ where $\theta$ is the moisture content, $K(h)$ is the hydraulic conductivity, $t$ is time, $x$ is horizontal distance, and $z$ is vertical distance. Data for the moisture capacity and the unsaturated hydraulic conductivity are presented on Figures $3.9 \mathrm{a}$ and $3.9 \mathrm{~b}$, respectively. Vauclin et al. (1979) estimate that the relationship:

$$
\theta(h)=\frac{\theta_{S}}{1+15.774|\mathrm{~h}|^{2.9}}
$$

where $\theta_{S}$ is the saturated moisture content (porosity), describes the moisture content fairly well. Furthermore, they estimate that:

$$
K(h)=\frac{K_{S}}{1+3344.48|h|^{5}}
$$

where $\mathrm{K}_{\mathrm{s}}$ is the saturated hydraulic conductivity, describes the hydraulic conductivity-capillary head relationship. Both curves are shown on Figures $3.9 \mathrm{a}$ and $3.9 \mathrm{~b}$. The saturated moisture content and hydraulic conductivity are 0.30 and $9.7222 \times 10^{-5} \mathrm{~m} / \mathrm{s}(35 \mathrm{~cm} / \mathrm{hr})$, respectively.

Several boundary conditions are applied to the modeled right portion of the slab. Both the lower boundary of the slab and the line of symmetry have zero flux boundary conditions. Along the top boundary, between $x=0.0$ and $x=$ $0.5 \mathrm{~m}$, a flux of $4.11 \times 10^{-5} \mathrm{~m} / \mathrm{s}$ into the slab is applied. Between $\mathrm{x}=$ 0.5 and $x=3.0 \mathrm{~m}$ along the top, and between $z=0.65 \mathrm{~m}$ and $z=2.00 \mathrm{~m}$ along the right side of the slab, a seepage face boundary condition is applied. A seepage-face boundary is a mixed boundary condition in that, if the soil is saturated, the total head is equal to the elevation head, and, if the soil is unsaturated, the water flux is zero.

Two types of initial conditions are applied to the slab interior. In the saturated zone, between $z=0.0$ and $0.65 \mathrm{~m}$, the total head is fixed at 0.65 $\mathrm{m}$. Above $z=0.65 \mathrm{~m}$, in the unsaturated zone, there is no water flux.

Code output in the form of moisture content with respect to depth is required at $x=0.19,0.49,0.79,1.09,1.39,1.69$ and $1.99 \mathrm{~m}$ from the line of symmetry, and $t=1800,3600,5400,7200,10800,14400,18000,21600$, and $28800 \mathrm{~s}$.

\subsubsection{Convection Cell}

The convection cell experiment is described, performed, and modeled by Reda (1984). In this experiment, the annular region between two vertical concentric cylinders is filled with a saturated porous medium and heated. The application of heat creates forced convection cells in the porous medium which convect heat throughout the annular region. A schematic of this experiment is presented on Figure 3.10. The purpose of modeling this 


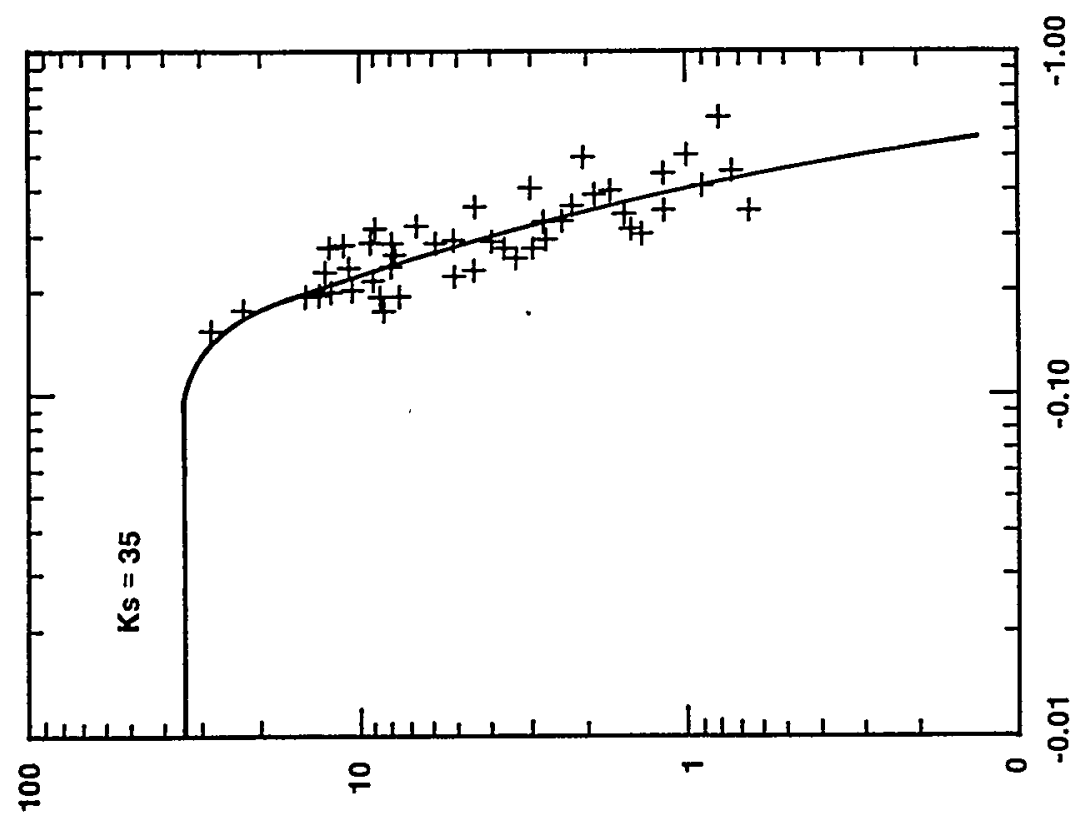

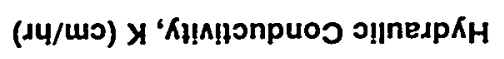

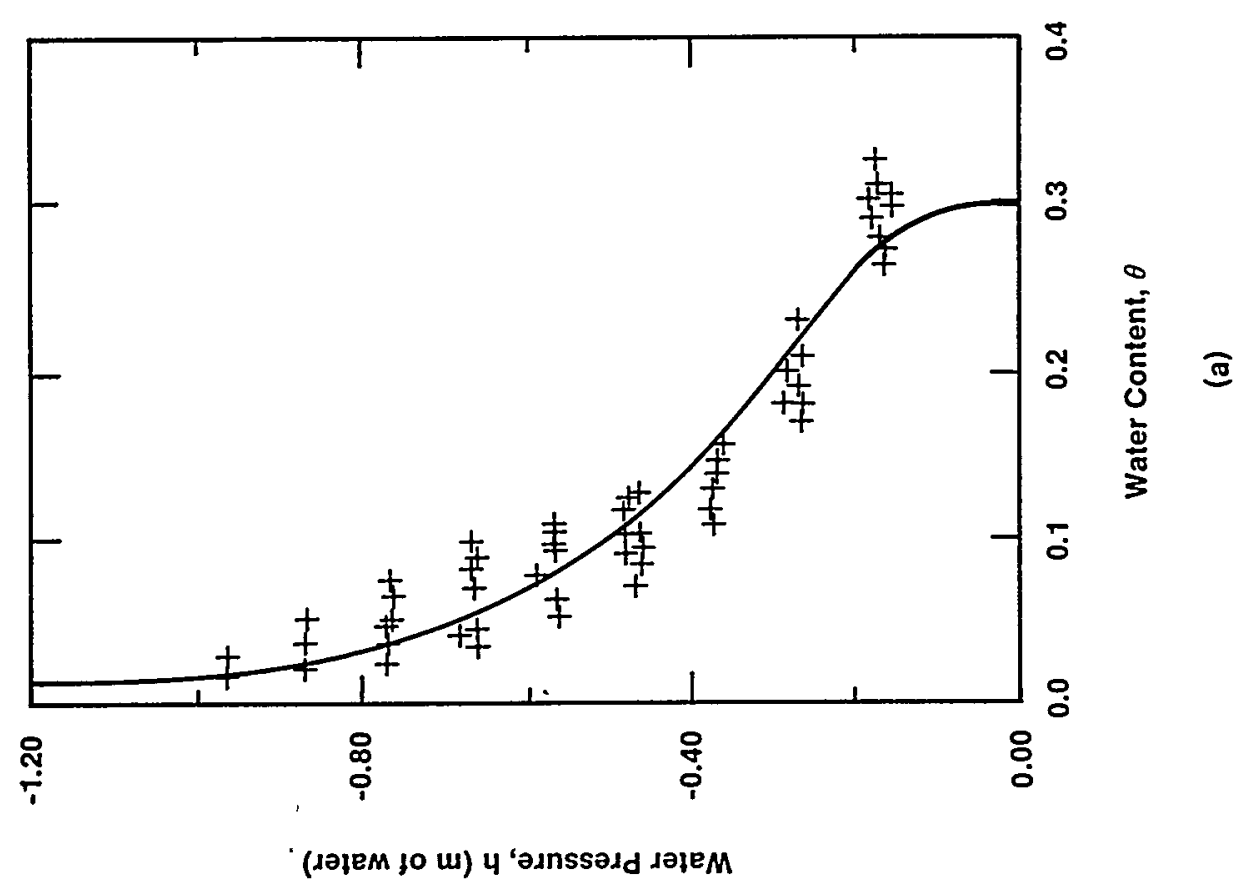

吾

要

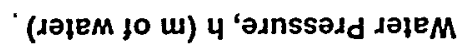




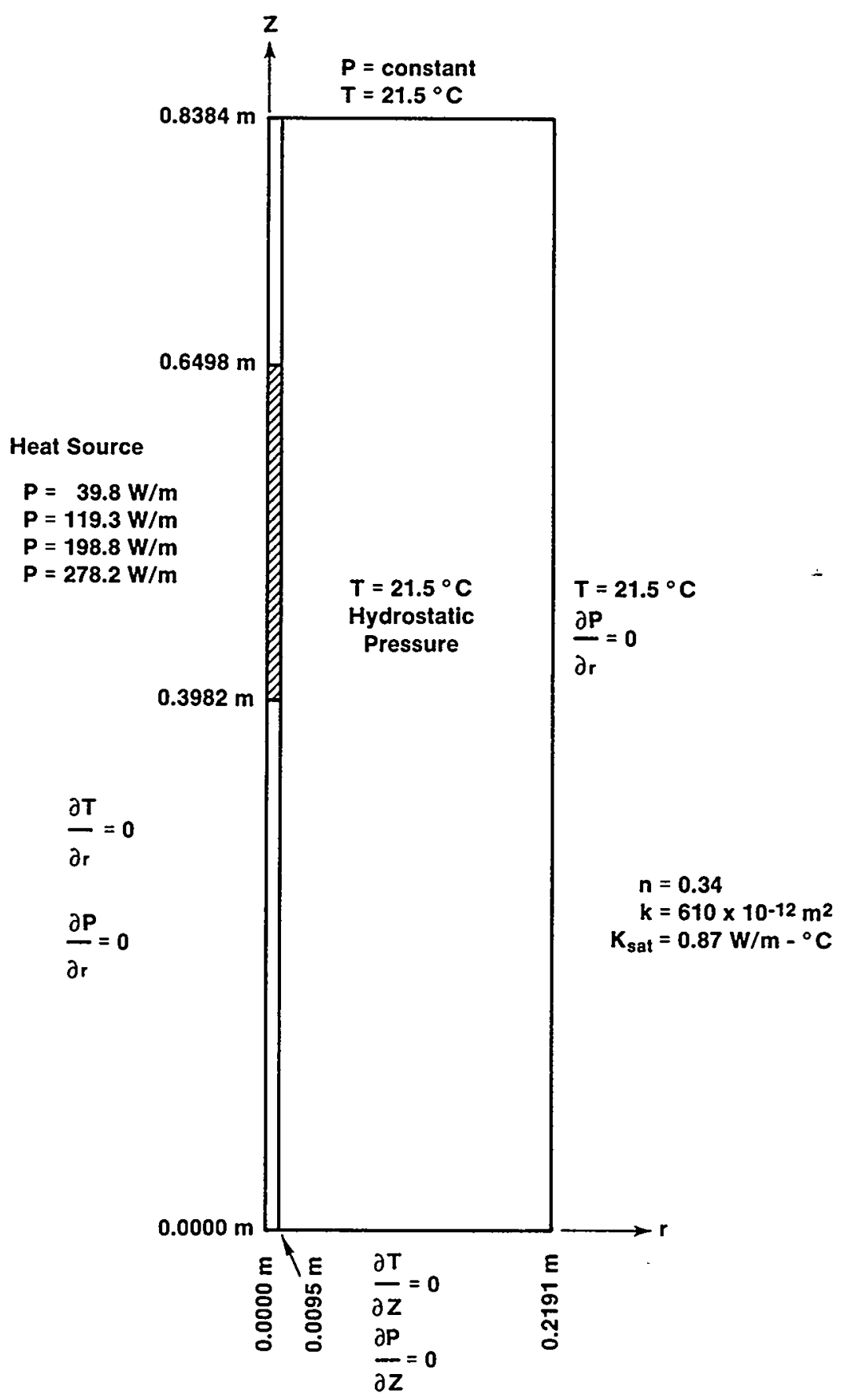

Figure 3.10: Convection Cell Problem 
experiment was to test the various codes' capabilities to handle multidimensional problems and simulate heat transport.

The inner edge of the annular region consisted of a finite-length heat source supported above and below by an insulating rod of radius $0.0095 \mathrm{~m}$. Power is supplied through the heat source between $0.3982 \mathrm{~m}$ and $0.6498 \mathrm{~m}$ above the bottom of the annular region. The outer edge of the annular region consists of a constant temperature reservoir separated from the annular region by a thin wall. The thin wall effectively prevents water flux out the annular region in the radial direction. The bottom of the annular region is comprised of an insulated end plate that effectively prevents water and heat flux out this end. A constant-temperature water bath comprises the upper boundary of the annular region. The water bath has a fixed pressure and allows water either to enter or to leave the annular region during the experiment. Prior to heating, the water in the annular region was stagnant and had a fixed temperature. Figure 3.10 also shows the boundary conditions applicable to the experiment.

Parameters necessary to simulate the experiment include:

\author{
porosity \\ permeability \\ saturated thermal conductivity \\ rock grain density \\ rock grain specific heat
}

$$
\begin{aligned}
& 0.34 \\
& 610 . \times 10^{-12} \mathrm{~m}^{2} \\
& 0.87 \mathrm{~W} / \mathrm{m}-{ }^{\circ} \mathrm{C} \\
& 2500 \mathrm{~kg} / \mathrm{m}^{3} \\
& 740 \mathrm{~J} / \mathrm{kg}-{ }^{\circ} \mathrm{C} .
\end{aligned}
$$

Code output is required at elevations of $0.4192,0.6288,0.7336$, and $0.7860 \mathrm{~m}$ above the bottom of the annular region for various times between 100 and $100000 \mathrm{~s}$ for a power level of $278.3 \mathrm{~W} / \mathrm{m}$ at the level of the heater.

\subsubsection{Two-Phase Flow}

The two-phase flow experiment was performed and modeled by Kruger and Ramey (1974) and independently modeled by Faust and Mercer (1979). The experiment consisted of heating a pressurized core sample saturated with hot water. A diagram of the experiment is presented in Figure 3.11. This problem was used to test the codes' capability to handle vaporization of water and heat transport.

A saturated piece of core with a length of $0.5969 \mathrm{~m}$ and a cross-sectional area of $2.027 \times 10^{-3} \mathrm{~m}^{2}$ is placed in an oven and heated to $198.9^{\circ} \mathrm{C}$. The left end of the core is insulated to prevent the flux of heat, liquid, and vapor. The right end of the core has a valve which is slowly opened to the atmosphere such that the pressure at the right side decreases with time. The pressure decline can be described approximately by:

$$
P_{\Upsilon}=1840965.0-11395.51972 t+21.4064213 t^{2}
$$


$T=198.9^{\circ} \mathrm{C}$

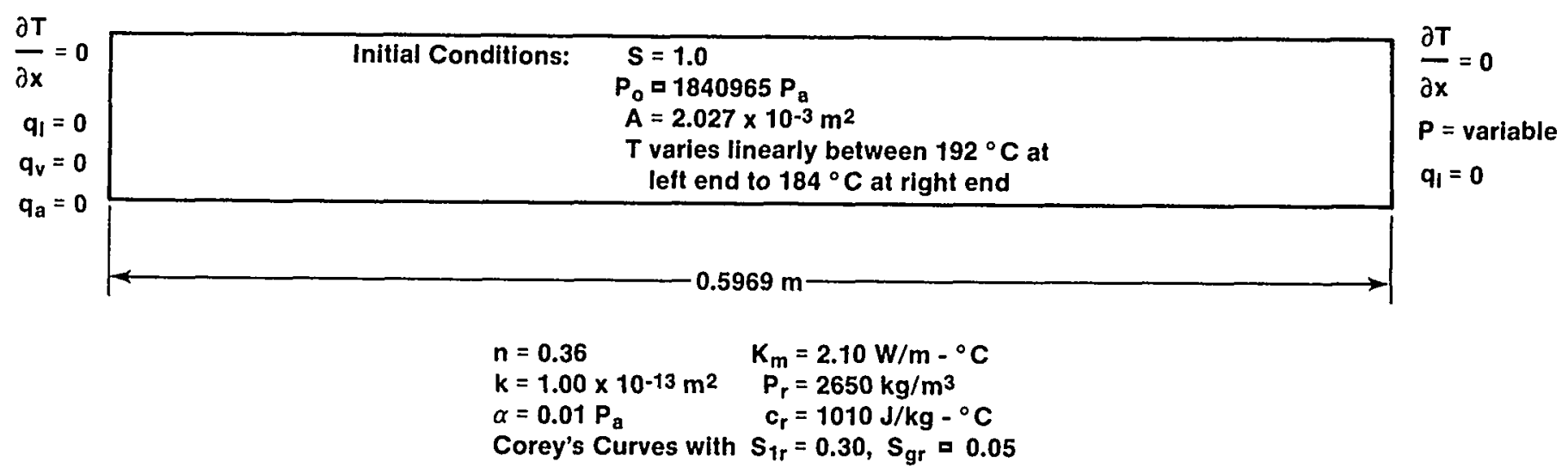

Figure 3.11: Two-Phase Flow Problem 
CHAPTER 4

CODE COMPARISONS

This section presents comparisons of the various codes with exact and numerical solutions or experimental results for the problems described in Section 3. Comparisons with exact and numerical solutions are presented first. For the most part the strongly heat-driven flow codes produced good comparisons with the exact or numerical solutions for the simpler problems. For the more complex solutions, those involving vaporization, the codes produced poor solutions or no solutions could be obtained. Comparison with the experiments are presented in the second part of this section. For the most part, solutions with the codes for comparison with experimental results could not be obtained.

\subsection{Comparison With Exact or Numerical Solutions}

\subsubsection{One-Dimensional Horizontal Infiltration}

\subsubsection{TOUGH}

TOUGH successfully ran the one-dimensional horizontal infiltration problem to completion. The code required approximately $12.1 \mathrm{~s}$ of $\mathrm{CPU}$ time to complete the problem. The grid used in the solution to the problem is shown in Figure 4.1. The grid consisted of a front row of grid blocks labelled LB1 and F1 through F40, and a back row labelled R1 through R40. The sole purpose of the back row was to suck air out of front-row grid blocks so that air would not interfere with the flow of water in the front row. The LB1 element, a boundary-condition element, was assigned an extremely large volume to keep the saturation at 1.000 at the left boundary. Grid blocks F1 through F40 were given widths of $0.005 \mathrm{~m}$ and heights and depths of $1.0 \mathrm{~m}$. Heights and depths are irrelevant to the solution of one-dimensional problems with TOUGH, but they need to be specified anyway. Zero flux boundary conditions are implied along the right edge of block $R 40$ because no additional grid blocks are attached there. The initial time step was 1.0 seconds, which was allowed to double if the solution at a time step converged within four iterations. The code required 28 time steps to complete the solution.

Results of the run for times of 864,5184 , and 9504 seconds are presented in Figure 4.2. The simulation results compare very well with the exact solution results for saturations greater than approximately 0.7 . Below this limit, the simulation results are greater than the exact solution by less than 58 of the exact solution. The results from TOUGH follow the trend of the exact solution very closely. In general, there is good agreement between the results from TOUGH and the exact solution. 


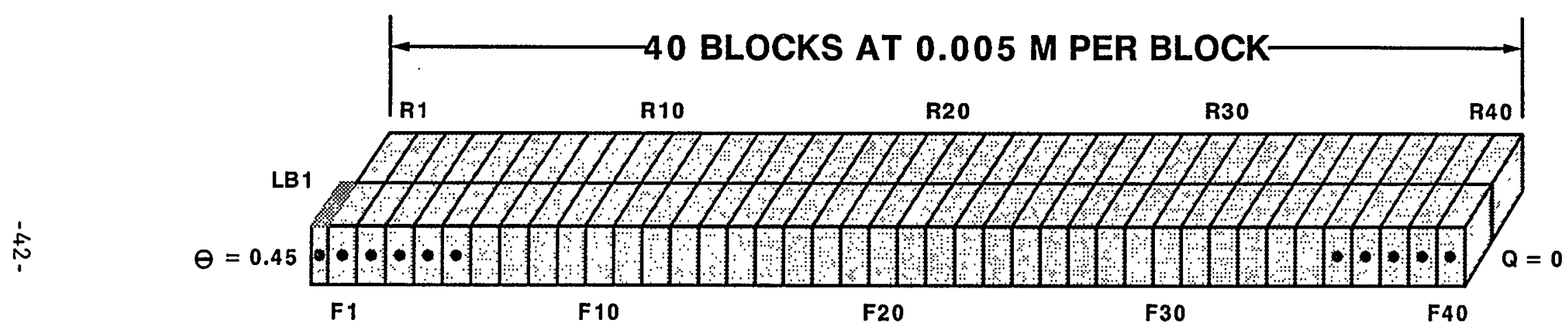

INITIAL CONDITION: $\theta=0.20 \ldots$ 


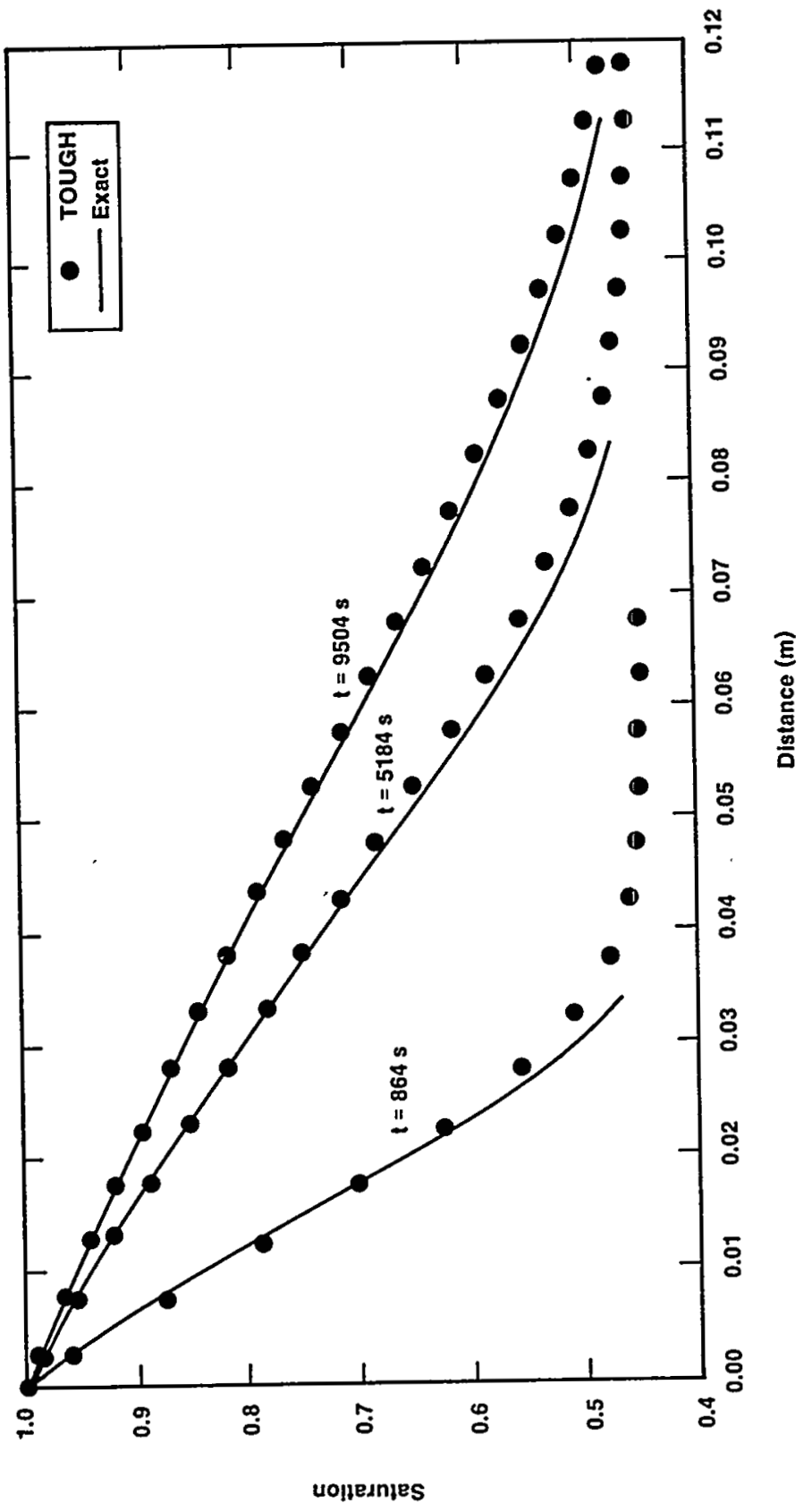

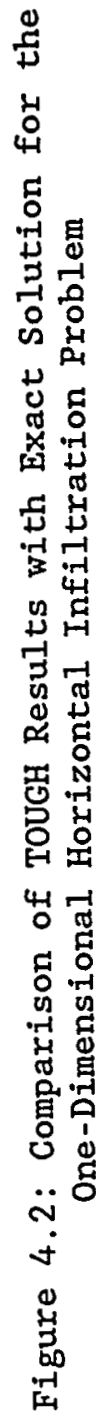




\subsubsection{NORIA}

NORIA successfully ran the one-dimensional horizontal infiltration problem but required approximately $1980 \mathrm{~s}$ of CPU time to do so. The grid used to model this problem is presented in Figure 4.3. It consisted of 20 finite elements, each $0.0100 \mathrm{~m}$ wide and $0.0001 \mathrm{~m} \mathrm{high}$. The height of the elements is irrelevant to the solution of the problem, but comparisons with various heights show that the solution converged better with a height this small.

The problem was modeled with Dirichlet boundary conditions along the left side of element 1 and the right side of element 20. The liquid pressure along the left side was set to atmospheric pressure, $101330 \mathrm{~Pa}$, to insure full saturation at that point. The liquid pressure at the right side of element was fixed at $93157.5 \mathrm{~Pa}$ to reflect the initial condition saturation of 0.4444 . The vapor and air pressures were arbitrarily fixed at $10000 \mathrm{~Pa}$ and $91330 \mathrm{~Pa}$, respectively, because NORIA requires some type of pressure for gases or it produces zero divide errors. Finally, the temperature at both boundaries was fixed at $20^{\circ} \mathrm{C}$. The initial time-step size was 0.01 seconds and was allowed to increase or decrease with time according to NORIA's built-in time-step-size criteria. The code required 935 time steps to complete the simulation.

The results from NORIA for times of 864,5184 , and $9504 \mathrm{~s}$ are presented in Figure 4.4. There is an excellent agreement between the resuts from NORIA and the exact solution. There is no noticeable difference between the two solutions at any time or distance. The use of quadratic element basis functions, which produce a closer approximation to the exact solution than linear basis functions, and small time steps probably account for the excellent agreement between solutions.

\subsubsection{PETROS}

PETROS successfully ran the one-dimensional horizontal infiltration problem. Three runs were necessary, each with a different ending time of 864,5184 , and $9504 \mathrm{~s}$; the required CPU times were approximately $2.0,2.9$, and $3.3 \mathrm{~s}$, respectively, and the required time steps were 131,198 , and 221 , respectively.

The PETROS grid for modeling the one-dimensional horizontal infiltration problems is shown in Figure 4.5. The grid consists of 41 nodes, each separated by a distance of $0.005 \mathrm{~m}$. Nodes at each end of the grid, nodes 1 and 41, are located on the boundaries. The left boundary was held fixed at a saturation of 1.00 and a temperature of $20^{\circ} \mathrm{C}$, and the right boundary was fixed at the initial conditions, a saturation of 0.4444 and a temperature of $20^{\circ} \mathrm{C}$. The air and vapor phase pressure equations were decoupled from the problem and were not solved. The initial time step was chosen as $1.0 \mathrm{~s}$ and allowed to vary according to the criteria in PETROS.

The results from PETROS compared to the exact solution are presented in Figure 4.6. For times of 864 and $5184 \mathrm{~s}$, there is an excellent agreement between the two solutions for saturations greater than 0.8 over the distance simulated. Below saturations of 0.8 , PETROS predicts saturations slightly less than the exact solution. The differences are less than approximately 


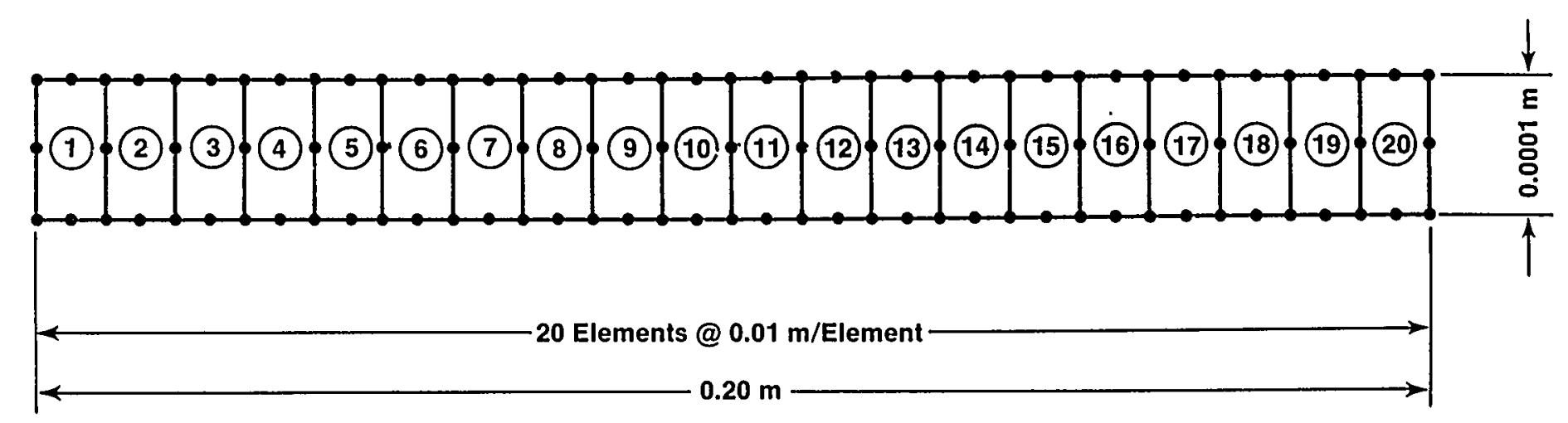

Figure 4.3: NORIA Grid for the One-Dimensional Horizontal Infiltration Problem 


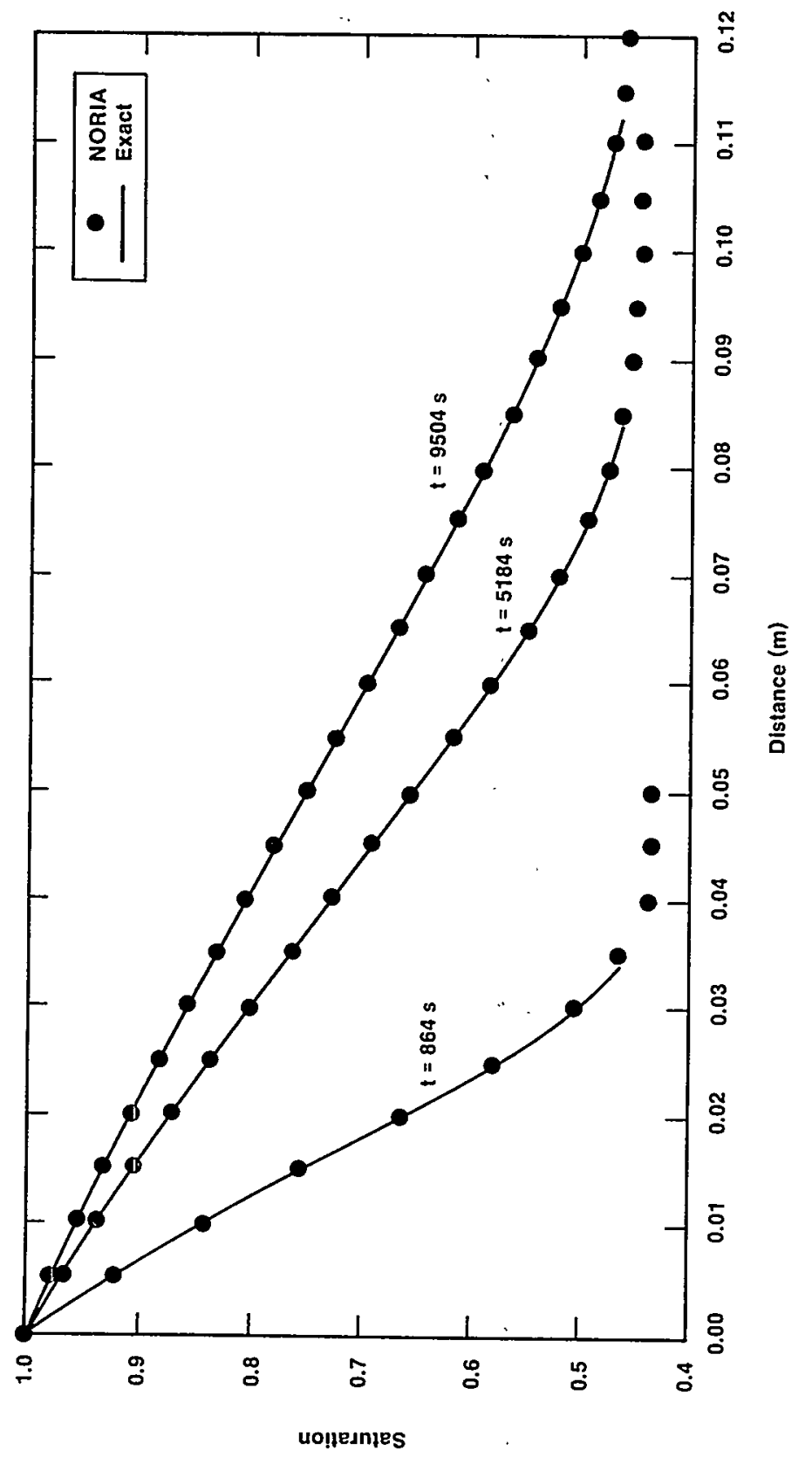

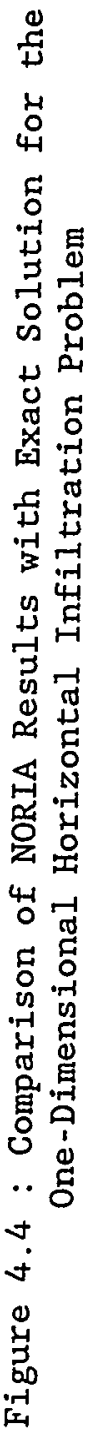




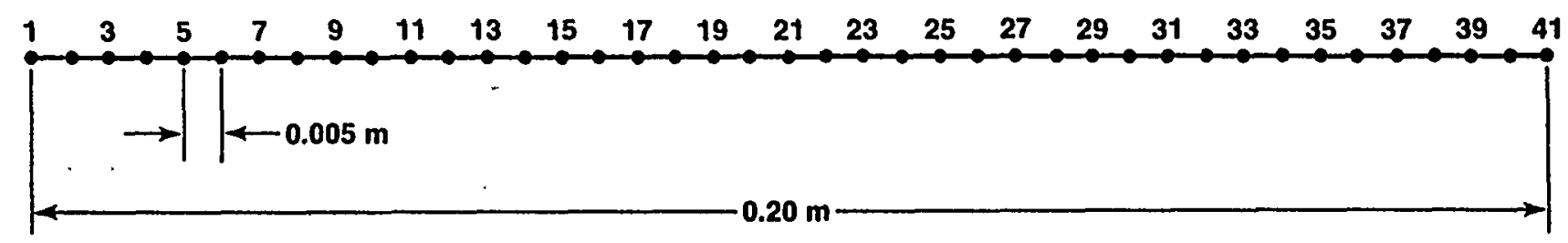

Figure 4.5: PETROS Grid for the One-Dimensional Horizontal Infiltration Problem 


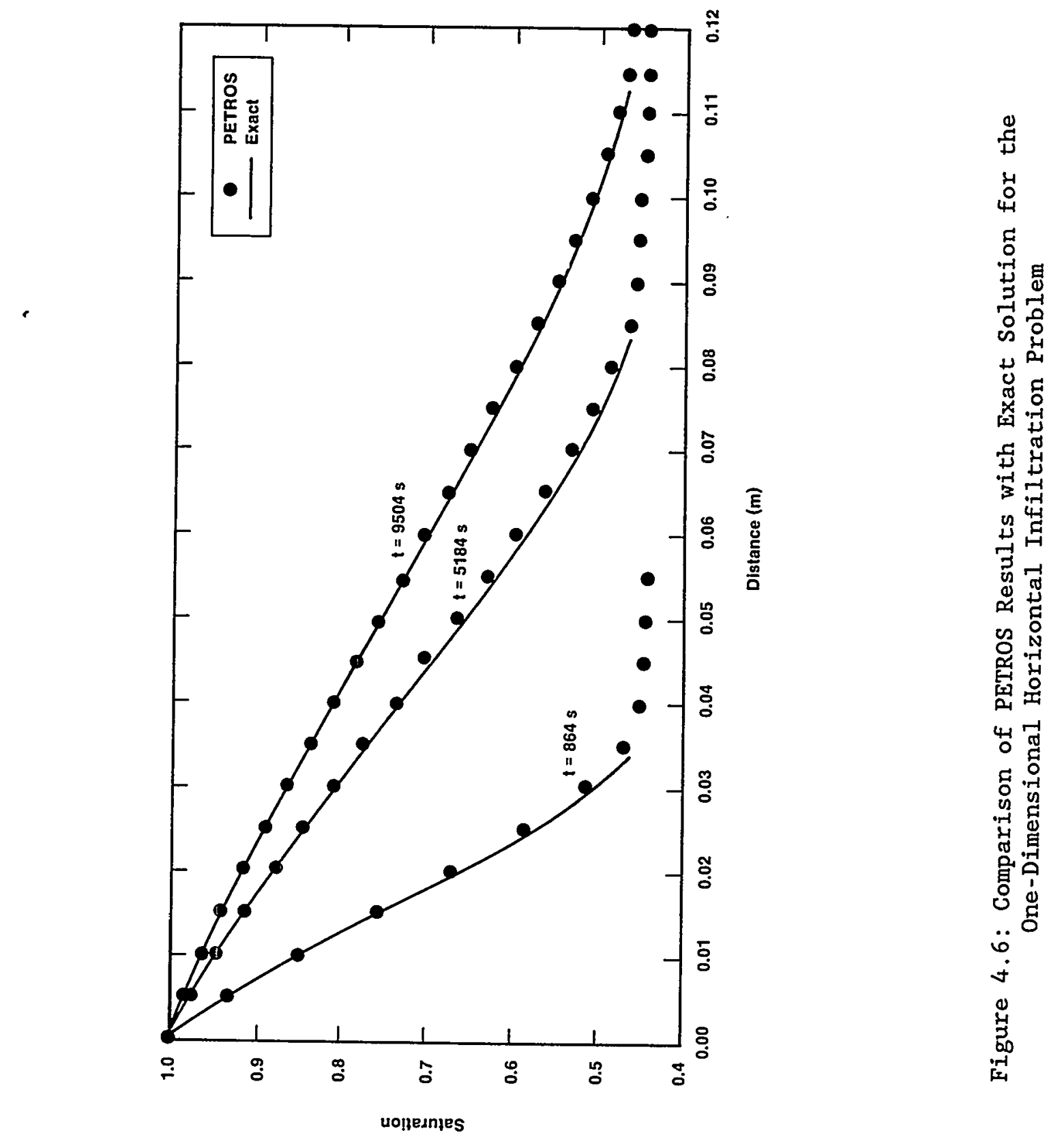


28. For a time of 9504 s, PETROS agrees with the exact solution extremely we11. It appears that, as time increases, the comparison between PETROS and the exact solution gets better. Overall, there is a very good comparison between the two solutions.

\subsubsection{One-Dimensional Heat Transport}

\subsubsection{TOUGH}

TOUGH successfully ran the linear heat transport problem, but required approximately 967 seconds of CPU time and 1300 time steps to do so. The grid used to solve the problem is presented in Figure 4.7. The grid consisted of 502 grid blocks. The left grid block, LBI, and the right grid block, RB1, were each assigned very large volumes to keep the left and right boundary pressures and temperatures constant throughout the simulation. The left boundary grid block was held fixed at a temperature of $160^{\circ} \mathrm{C}$ and a pressure of $5050000 \mathrm{~Pa}$ and the right boundary grid block at $170^{\circ} \mathrm{C}$ and 5 $000000 \mathrm{~Pa}$. The initial temperature was set at $170^{\circ} \mathrm{C}$. The initial pressure varied linearly between $5050000 \mathrm{~Pa}$ and $5000000 \mathrm{~Pa}$ throughout the modeled region. The permeability of $1.74 \times 10^{-9} \mathrm{~m}^{2}$, the viscosity of water for a temperature between $160^{\circ} \mathrm{C}$ and $170^{\circ} \mathrm{C}$, and the pressure gradient kept the mass flux of water at $10 \mathrm{~kg} / \mathrm{s}$ within the modeled region. The choice of the permeability and the pressure gradient could be anything as long as they are chosen to maintain the proper flow rate. The grid spacing of $0.1 \mathrm{~m}$ and time-step size of $100 \mathrm{~s}$ were based on criteria established in the SWIFT II documentation (Reeves et al., 1986) to control numerical dispersion and overshoot. However, a limiting factor in determining the grid spacing was dimensions of certain arrays in the code.

A comparison of TOUGH results with the exact solution is presented on Figure 4.8. The results indicate that, although the location of the heat front predicted with TOUGH appears to be located at the same distance as the location obtained with the exact solution, some numerical dispersion occurs. Apparently, the grid spacing and time-step sizes were too large, which were restricted by the memory requirements in TOUGH.

\subsubsection{NORIA}

The NORIA code successfully simulated the linear heat transport problem with approximately $3850 \mathrm{~s}$ of GPU time. The finite-element grid used to solve the problem with NORIA is presented on Figure 4.9 . Only $30 \mathrm{~m}$ of the aquifer were modeled so the grid consisted of 150 elements each $0.2 \mathrm{~m}$ wide. Node spacing was $0.1 \mathrm{~m}$, the same as that used with TOUGH for this problem. An initial time step of $0.1 \mathrm{~s}$ was used to start the simulation and the time-step size increased with simulation time according to the criteria in NORIA. The code required 225 time steps to solve the problem with the final time-step size being $1195 \mathrm{~s}$.

The left boundary was held fixed at a temperature of $160^{\circ} \mathrm{C}$ and a liquid pressure of $5050000 \mathrm{~Pa}$. The right boundary was held fixed at a temperature of $170^{\circ} \mathrm{C}$, the initial temperature, and a pressure of $5000000 \mathrm{~Pa}$. The 


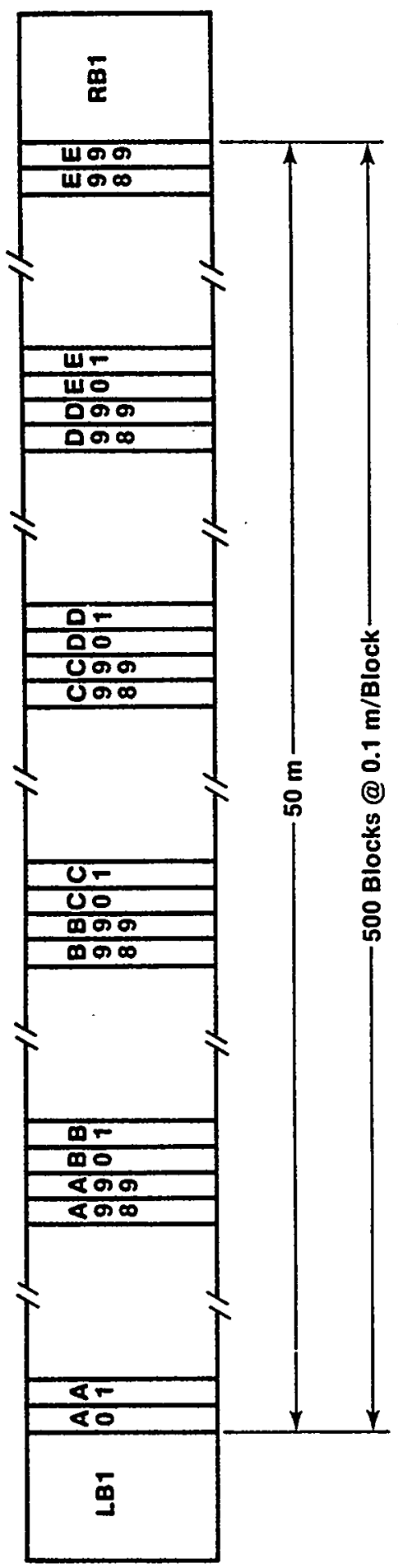

章 


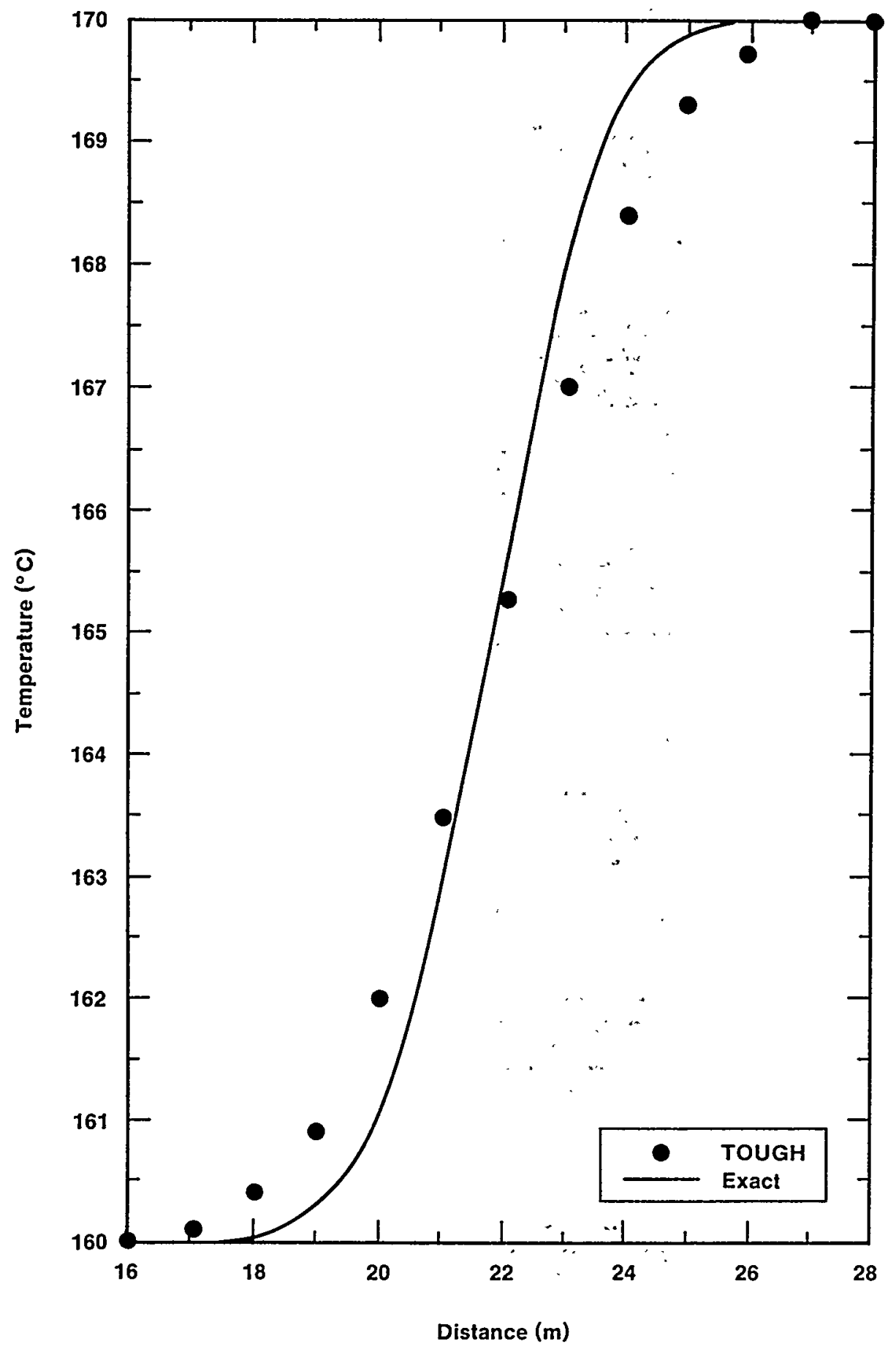

Figure 4.8: Comparison of TOUGH Results with Exact Solution for the One-Dimensional Heat Transport Problem 


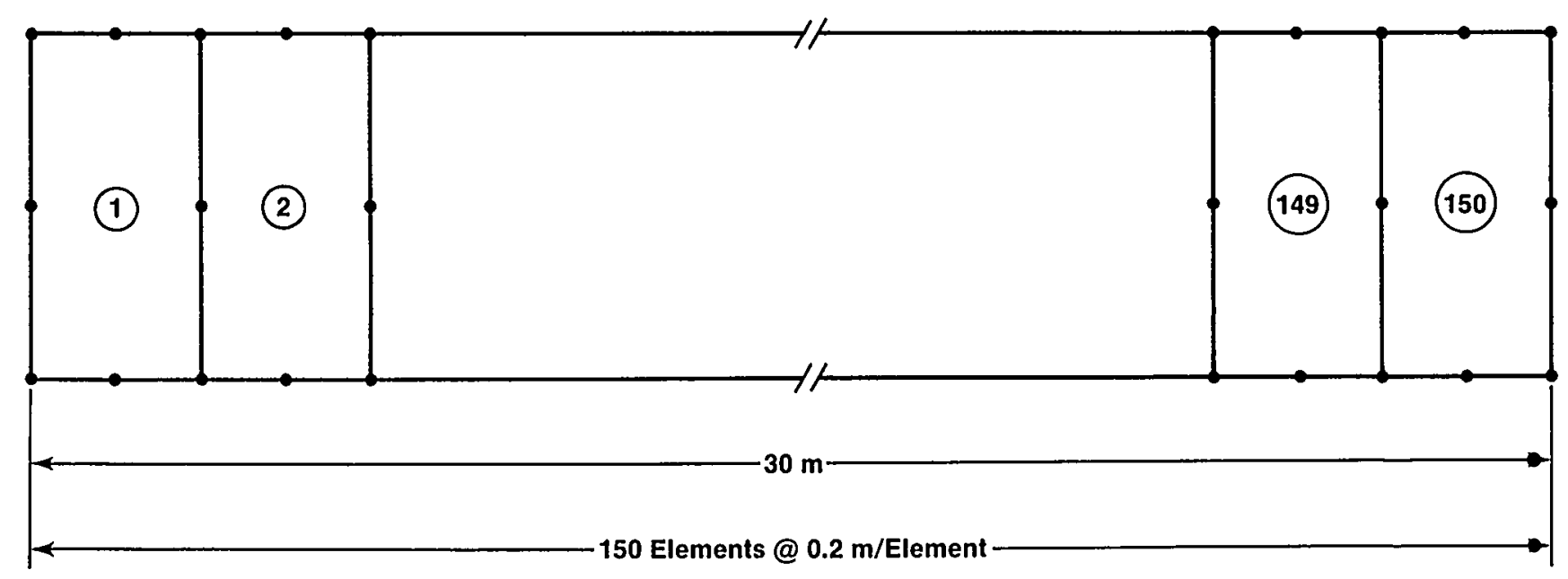

Figure 4.9: NORIA Grid for the One-Dimensional Heat Transport Problem 
initial pressure varied linearly between the assigned boundary pressures at each end of the modeled region. The pressure gradient coupled with a constant permeability and viscosity of $1.09 \times 10^{-10} \mathrm{~m}^{2}$ and 0.001

$\mathrm{Pa} \cdot \mathrm{s}$, respectively, maintained a flow rate of $10 \mathrm{~kg} / \mathrm{s}$ through the aquifer. In addition, NORIA required initial and boundary values of the air and vapor pressure. These were both set arbitrarily to values of $25000 \mathrm{~Pa}$ throughout the modeled region.

A comparison of the NORIA solution with the exact solution is presented in Figure 4.10. There is a very good agreement between the two solutions. There is no evidence of numerical dispersion. Any difference between the two solutions seems to be caused by the location of the two fronts. The location of the midpoint of the front predicted by NORIA lags behind the midpoint of the front predicted by the exact solution by approximately $0.15 \mathrm{~m}$ or approximately 0.78 of the distance traveled.

\subsubsection{PETROS}

PETROS, needing 935 seconds of CPU time, successfully solved the linear heat transport problem. The finite-difference grid used to solve the problem with PETROS is presented in Figure 4.11. The grid consisted of 501 grid points, $0.1 \mathrm{~m}$ apart. Grid points 1 and 501 are located on the boundaries of the modeled region. An initial time-step size of $1.0 \mathrm{~s}$ was used to start the simulation. Time-step sizes varied up to a maximum of $100 \mathrm{~s}$ according to the time-step criteria in PETROS. The simulation required 1317 time steps.

The left boundary was held fixed at a saturation of 1.0 , a temperature of $160^{\circ} \mathrm{C}$, and a vapor pressure of $5050000 \mathrm{~Pa}$. Similarly, the right boundary was held fixed at saturation of 1.0 , a temperature of $170^{\circ} \mathrm{C}$, and a vapor pressure of $5000000 \mathrm{~Pa}$. The air equation was not solved in this simulation. The initial conditions were set at a saturation of 1.0 and a temperature of $170^{\circ} \mathrm{C}$. The initial liquid pressure could not be set explicitly in PETROS. The gas pressure equations in PETROS do not include a time-dependent term and, therefore, an initial condition is not necessary for these equations. Instead, to set initial gas pressure conditions, PETROS solves the steady-state gas pressure equations based on the specified boundary conditions. In this case, the resulting gas pressure solution produced a linear gas pressure initial condition. By setting the capillary pressure to zero, the liquid pressure initial condition was the same as the gas pressure initial condition, a linear function with a pressure of $5050000 \mathrm{~Pa}$ at the left end and $5000000 \mathrm{~Pa}$ at the right end. The permeability of $1.09 \times 10^{-10} \mathrm{~m}$ and liquid dynamic viscosity of 0.001 $\mathrm{Pa} \cdot \mathrm{s}$ maintained a $10 \mathrm{~kg} / \mathrm{s}$ mass flow rate throughout the simulation.

A comparison of the PETROS solution with the exact solution is presented in Figure 4.12. There is a small amount of numerical dispersion noticeable on the figure. However, the location of the fronts produced by the two solutions is the same. Overall, there is a very good agreement between the PETROS and the exact solutions. 


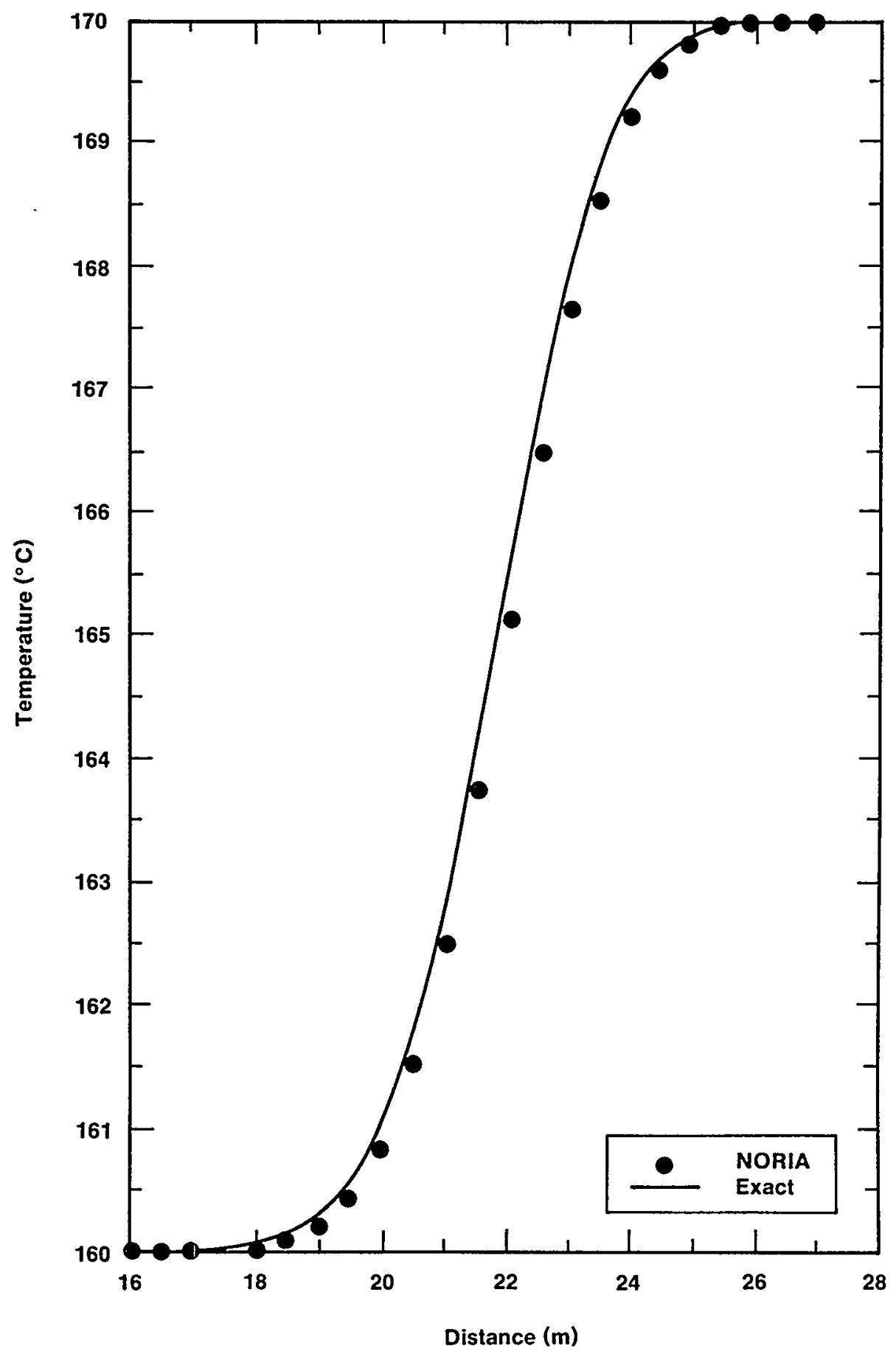

Figure 4.10: Comparison of NORIA Results with Exact Solution for the One-Dimensional Heat Transport Problem 

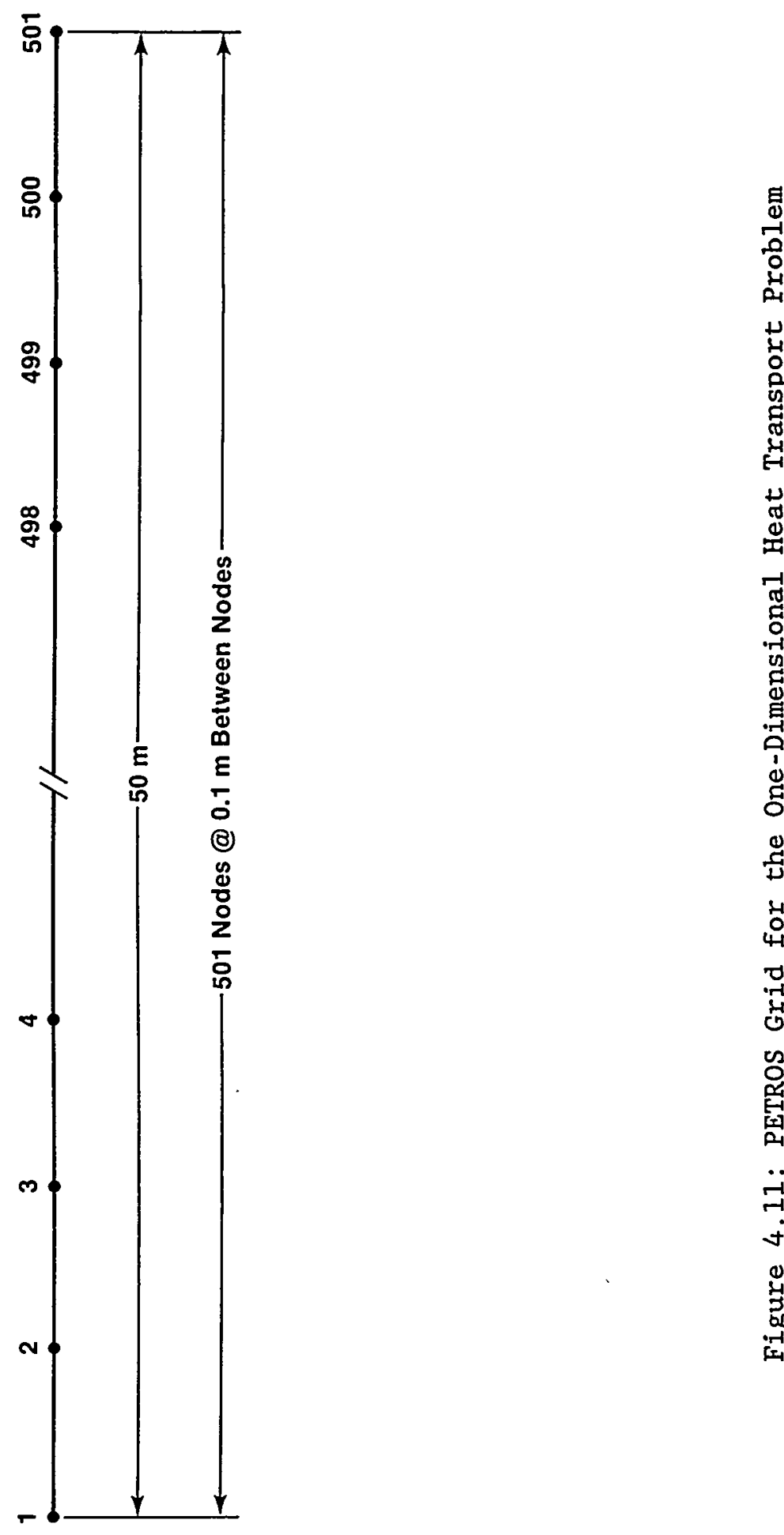

ษ

茫

劣

s

舅

P.

$\ddot{-}$

$\checkmark$

苛 


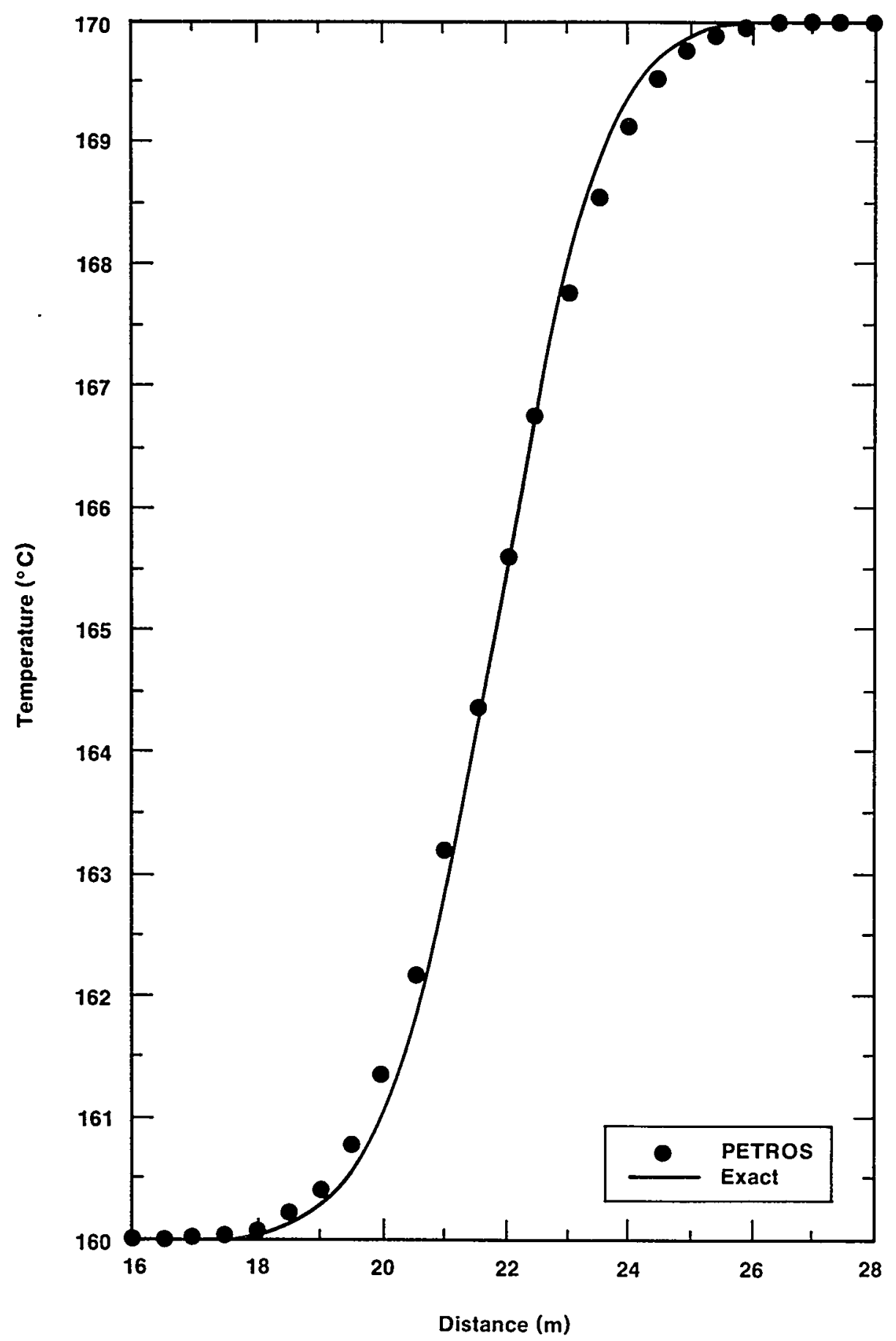

Figure 4.12: Comparison of PETROS Results with Exact Solution for the One-Dimensional Heat Transport Problem 


\subsubsection{Radial Heat Transport}

\subsubsection{TOUGH}

TOUGH could only simulate the first $1.5 \times 10^{6} \mathrm{~s}$ of the radial heat transport problem with $2000 \mathrm{~s}$ of CPU time and 3000 time steps. The problem specification required $1 \times 10^{\circ} \mathrm{s}$ or approximately 32 years of simulation. It was unlikely that TOUGH could have simulated the 32-year problem with $1 \mathrm{~h}$ of CPU time. For this reason, the TOUGH prediction is compared only with the exact solution at $1 \times 10^{6} \mathrm{~s}$.

The grid used to simulate this problem with TOUGH is shown in Figure 4.13. It consisted of 252 elements of which IBI and OBI were used to control the pressure and temperature boundary conditions. The IBI element represented the injection well and was assigned a large volume to hold the wellbore temperature and pressure fixed at $160^{\circ} \mathrm{C}$ and $5050000 \mathrm{~Pa}$, respectively, and a small radius of $0.01 \mathrm{~m}$ in order to approximate an infinitesimally small well. Subsequent distances to outer edges of grid blocks (i.e. A-1 through C-49) were assigned such that the ratio of the distance to the outer edge of. a grid block to the outer edge of the preceding grid block was 1.047128548 or $(1000 \mathrm{~m} / 0.01 \mathrm{~m})^{1 / 250}$, where $1000 \mathrm{~m}$ is the distance from the wellbore center to the outer edge of the aquifer, $0.01 \mathrm{~m}$ is the wellbore radius, and ' 250 ' is the number of grid blocks through which there is flow. This is the same relationship that SWIFTII uses to calculate the grid spacing for cylindrical coordinate systems (Reeves et al., 1986). The OB1 element, 1000 $\mathrm{m}$ away from the wellbore center, was assigned a large volume to hold the temperature and pressure fixed at $170^{\circ} \mathrm{C}$ and $5000000 \mathrm{~Pa}$, respectively. The initial temperature was set at $160^{\circ} \mathrm{C}$ and the initial pressure distribution was determined according to a logarithmic pressure function:

$$
P_{1}=P_{0}+\left(P_{i}-P_{0}\right) \frac{\ln \left(r / r_{0}\right)}{\ln \left(r_{i} / r_{0}\right)}
$$

where $P_{1}$ is liquid pressure, $P_{0}$ is pressure at the outer boundary, $P_{i}$ is pressure at the wellbore, $r$ is distance from the center of the wellbore, $r_{0}$ is distance between the center of the wellbore and the outer boundary, and $r_{i}$ is the radius of the wellbore. The pressure gradient determined from the above equation, the mass flux rate of $10 \mathrm{~kg} / \mathrm{s}$ at the wellbore, and the density and viscosity of water at $165^{\circ} \mathrm{C}$ (the midpoint of $160^{\circ} \mathrm{C}$ and $170^{\circ} \mathrm{C}$ ) resulted in a permeability of $6.36 \times 10^{-11} \mathrm{~m}^{2}$ being assigned to this problem.

A comparison of the TOUGH results to the exact solution results is presented in Figure 4.14. The comparison seems to indicate little numerical dispersion. However, the distance traveled by the front predicted by TOUGH is only about $95 \%$ of the distance predicted by the exact solution. It is suspected that the temperature dependence of the density and viscosity models implemented in TOUGH are the cause for this difference. As the cold water is injected into the aquifer, the viscosity of the aquifer decreases and the fluid moves slower. This may account for the difference in the front locations. 


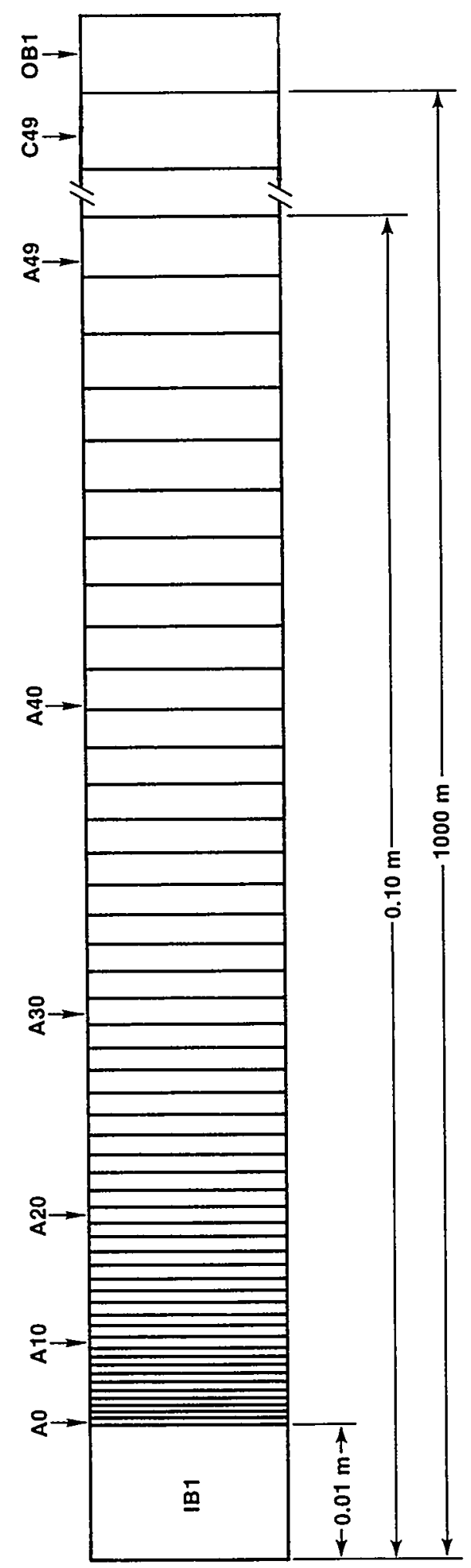

Oี 


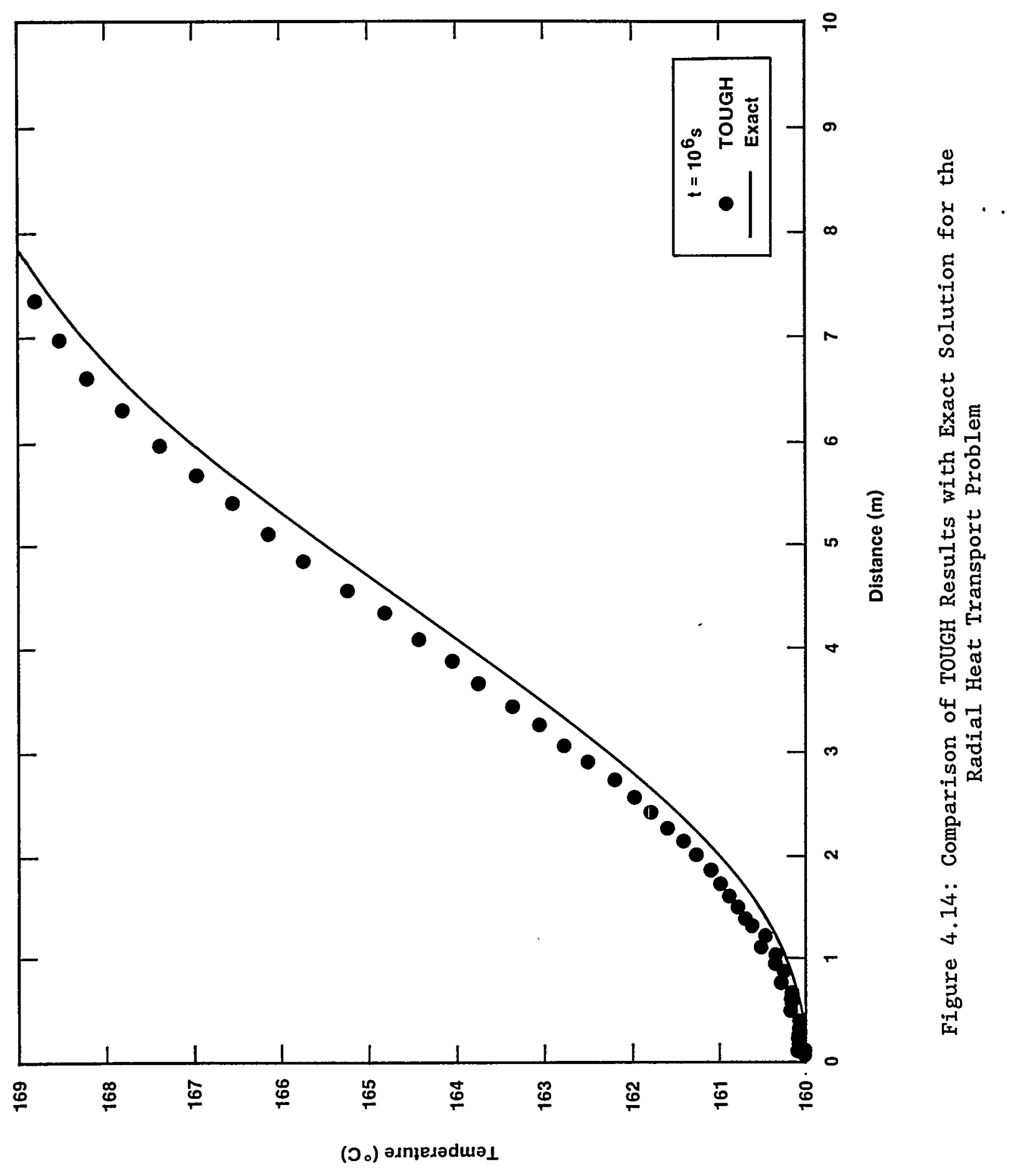




\subsubsection{NORIA}

NORIA could only simulate the first $4.6 \times 10^{6} \mathrm{~s}$ of the $1 \times 10^{9} \mathrm{~s}$ simulation for radial heat transport problem in $2 \mathrm{~h}$ of $\mathrm{CPU}$ time. The code required 459 time steps to solve the equations for the first $4.6 \times 10^{6}$ s. The initial time-step size was $1.0 \mathrm{~s}$. The time-step size selection behaved in an oscillatory manner throughout the simulation. The time-step size would be as large as $10^{5} \mathrm{~s}$, then reduce to $10^{2} \mathrm{~s}$, then increase again to $10^{5} \mathrm{~s}$.

A portion of the grid used to model the problem is presented in Figure 4.15. The exact solution indicated that the leading edge of the front would advance less than $560 \mathrm{~m}$ from the wellbore. Therefore, the outer edge of the grid was placed at $595.365 \mathrm{~m}$ from the wellbore. The grid consisted of 117 elements. Node spacing was specified such that the ratio of the distance between two successive nodes to the distance of the two preceding successive nodes was 1.032929909 . NORIA calculates the grid spacing based on the number of elements, the distance ratio, and the distance to the outer edge of the aquifer. From this information, NORIA calculated the distance between the first two nodes as $0.01 \mathrm{~m}$.

Constant pressure and temperature boundary conditions of $5050000 \mathrm{~Pa}$ and $160^{\circ} \mathrm{C}$ were assigned at the wellbore. At the outer boundary a constant pressure and temperature of $5002252 \mathrm{~Pa}$ and $170^{\circ} \mathrm{C}$, respectively, were assigned. The outer-boundary pressure condition was estimated from the pressure equation presented in the preceding section for a distance of $595.3596 \mathrm{~m}$. In addition, the initial temperature was $170^{\circ} \mathrm{C}$ and the initial pressure was estimated from the equation presented in the preceding section. The boundary and initial conditions for the air and vapor pressure equations, although irrelevant to the solution to the problem, were arbitrarily chosen as $2500000 \mathrm{~Pa}$.

Figure 4.16 presents a comparison of the NORIA solution with the exact solution at a time of $1 \times 10^{6} \mathrm{~s}$. No numerical dispersion appears and the location of the fronts generated by the two solutions is the same. Overall, there is excellent agreement between the NORIA and exact solutions.

\subsubsection{PETROS}

PETROS, requiring only $385 \mathrm{~s}$ of CPU time, was the only code to solve the radial heat transport problem for the required $1 \times 10^{9} \mathrm{~s}$ simulation time. Results for times of $1 \times 10^{6} \mathrm{~s}$ and $1 \times 10^{9} \mathrm{~s}$ are presented here. The $1 \times 10^{6} \mathrm{~s}$ case required only $28.3 \mathrm{~s}$ of CPU time.

A portion of the PETROS finite-difference grid is presented in Figure 4.17. The distance between the nodes is the same as in the NORIA grid. However, in contrast to the NORIA grid, the PETROS grid consists of 251 nodes, covering the range $r=0.01 \mathrm{~m}$, the wellbore radius, to $r=1000 \mathrm{~m}$, the distance to the outer boundary.

The saturation boundary conditions at each end of the grid and the initial conditions were specified as 1.0 since this was a saturated flow problem. The temperatures were specified as $160^{\circ} \mathrm{C}$ at the wellbore and $170^{\circ} \mathrm{C}$, 


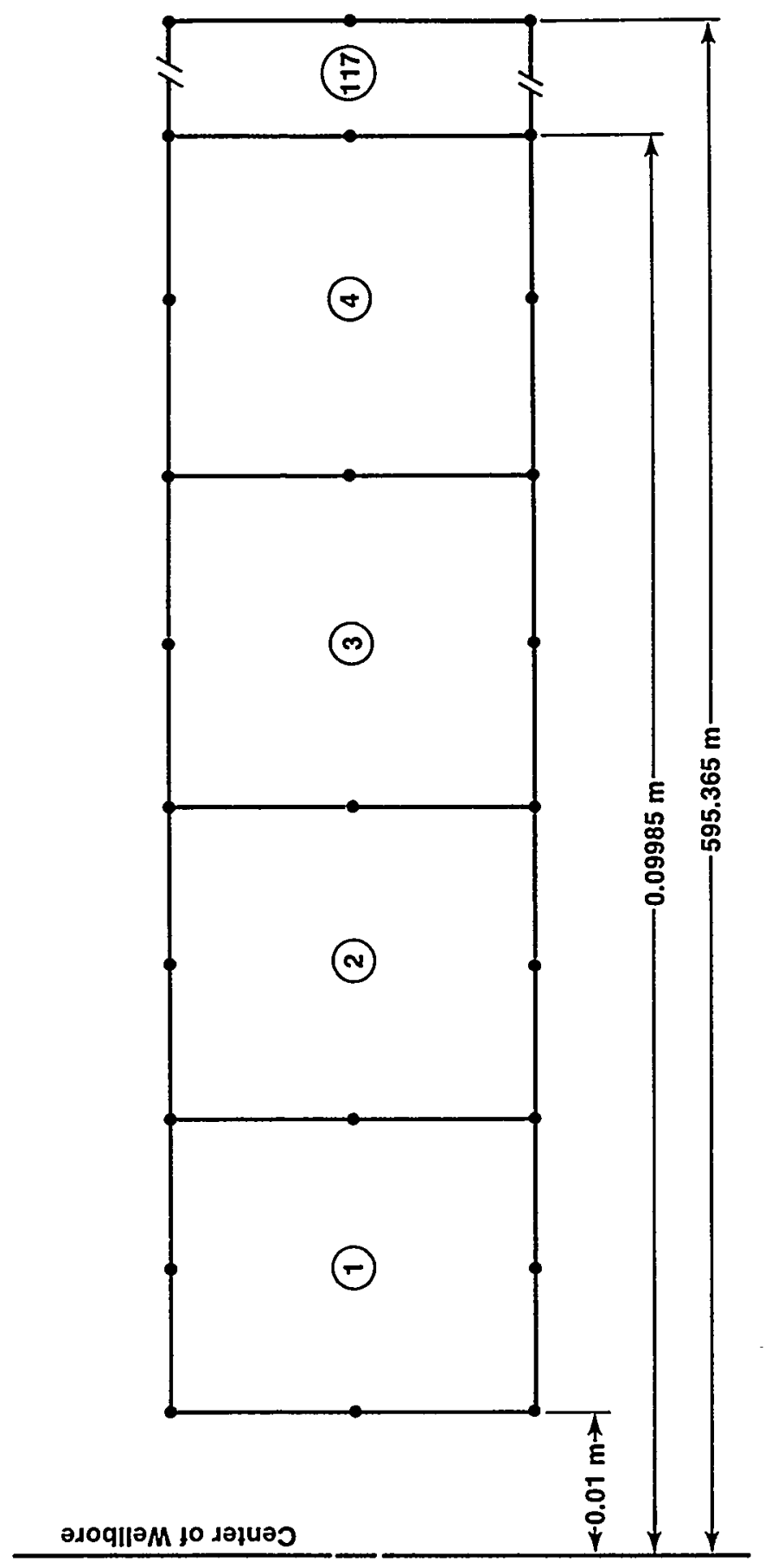

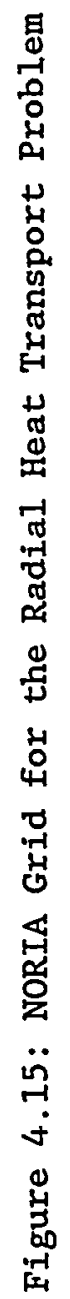




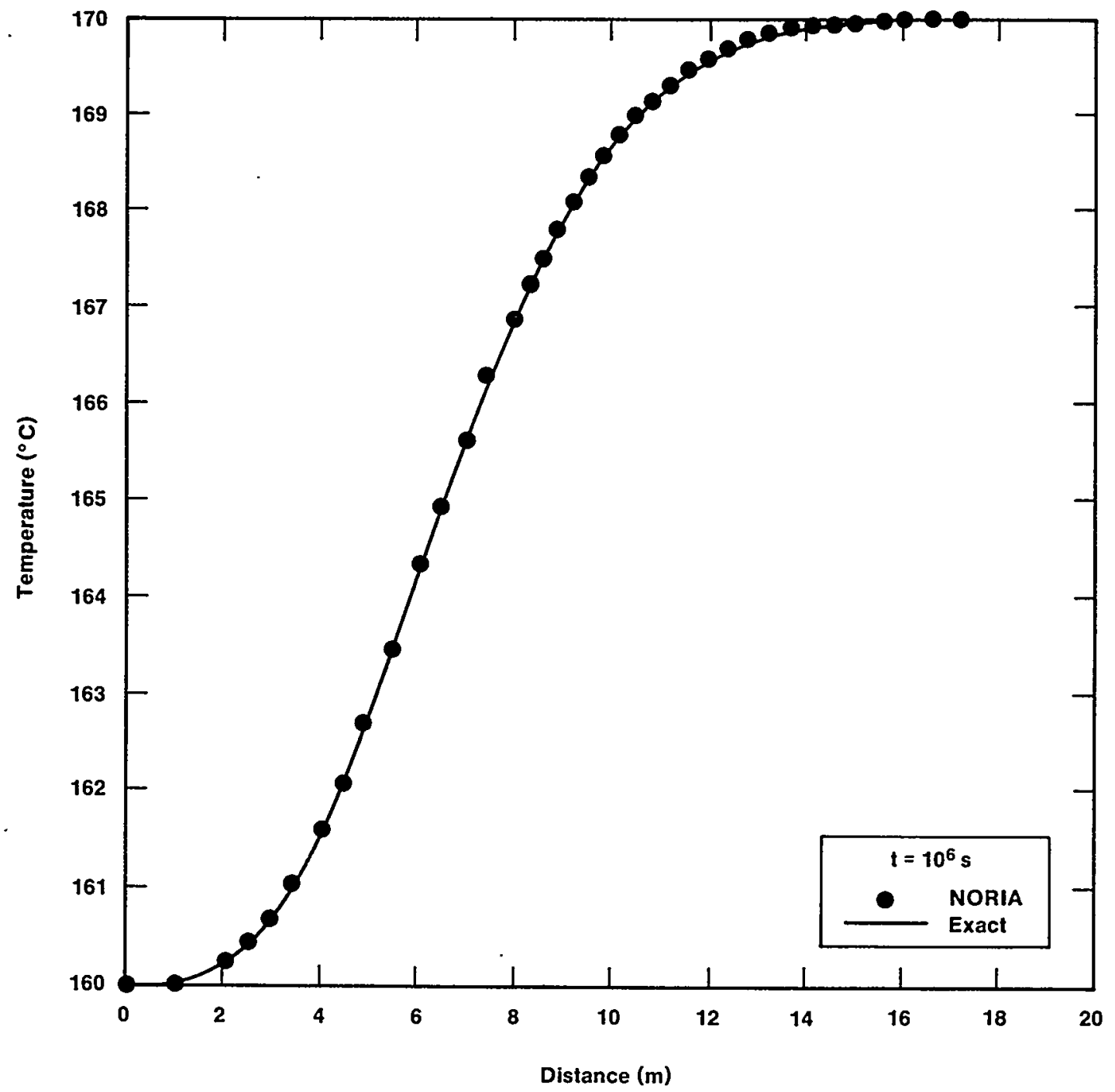

Figure 4.16: Comparison of NORIA Results with Exact Solution for the Radial Heat Transport Problem 


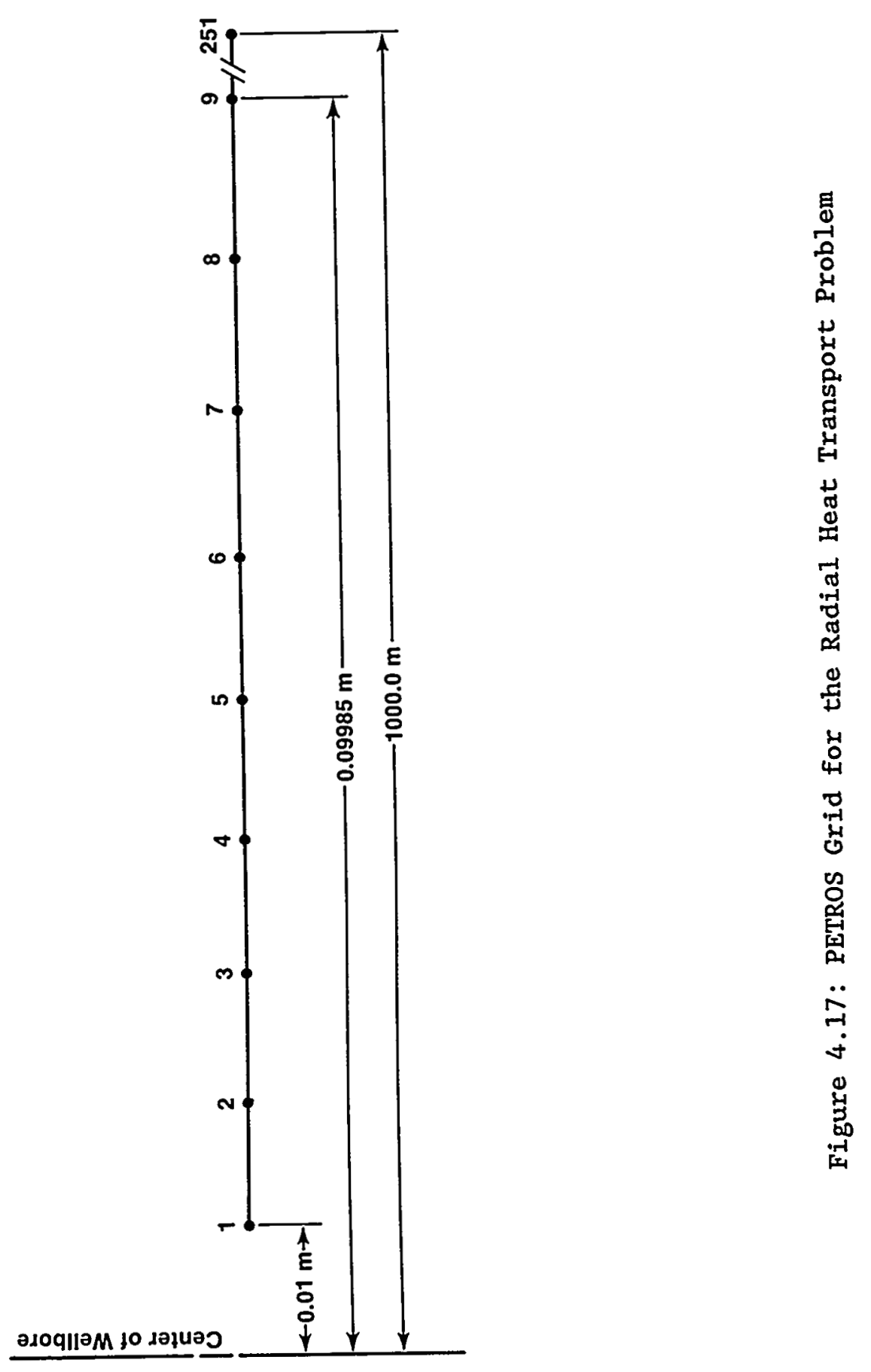

$-63-$ 
the initial condition, at the outer boundary. Finally, the vapor pressure was set to $5050000 \mathrm{~Pa}$ at the wellbore and $5000000 \mathrm{~Pa}$ at the outer boundary. As indicated in section 4.1.2.3, PETROS calculates the initial vapor pressure distribution. Since the capillary pressure is set to zero, the liquid pressure equals the vapor pressure and this equality sets up the initial liquid flow field. The initial time step for both runs was $1.0 \mathrm{~s}$. The $1 \times 10^{6} \mathrm{~s}$ run required 63 time steps and the $1 \times 10^{9} \mathrm{~s}$ run required 1066 time steps. Final time-step sizes for both runs were $77764 \mathrm{~s}$ and $471850 \mathrm{~s}$, respectively.

Results for both runs are presented on Figures 4.18 and 4.19 . For the $1 \times$ $10^{6} \mathrm{~s}$ run, the PETROS solution shows a small amount of numerical dispersion and the front advances slightly less than that predicted by the exact solution. The overall comparison is very good. For the $1 \times 10^{9} \mathrm{~s}$ run, the PETROS and the exact solutions compare extremely well. There is no evidence of any numerical dispersion and the locations of the fronts predicted by the two solutions coincide.

\subsubsection{Radial Boiling Front}

\subsubsection{TOUGH}

TOUGH, requiring 11.6 seconds of CPU time, successfully simulated the boiling front problem. A portion of the grid used to model the problem is presented in Figure 4.20. The grid consisted of 50 grid blocks, the first of which is the wellbore block where liquids and gases are removed. The first ten grid blocks, Al through $\mathrm{Al0}$, are each $1.0 \mathrm{~m}$ wide. The remaining 40 grid blocks are expanded such that the ratio of the width of one grid block to the width of the preceding grid block is approximately 1.149046286 . This ratio is a geometric ratio determined from the distance between the wellbore center to the outer edge of the grid, the grid spacing of the evenly spaced grid blocks, and the number of grid blocks in the unevenly spaced area. The distance from the center of the wellbore to the outer edge of the aquifer is approximately $1741.8 \mathrm{~m}$.

To force flow in the aquifer, the wellbore was treated as a sink from which mass is removed at a rate of $0.14 \mathrm{~kg} / \mathrm{s}$ per unit of aquifer thickness. At early times, the mass removed from the wellbore grid block consisted entirely of liquid. At later times, as the liquid in the aquifer vaporized, the mass removed from the wellbore consisted of both gas and liquid. The amount of gas and liquid withdrawn from the wellbore depended on the mobility ratio between the liquid and gas. This type of mass withdrawal is a user selected option in TOUGH. Zero flux conditions for both fluids and heat were assigned at the center of the wellbore and the outer edge of the aquifer.

A comparison of the TOUGH generated solution with Garg's (1980) numerical solution is presented on Figure 4.21. Except for the first 10 seconds of the simulation, the TOUGH solution agrees extremely well with Garg's numerical solution. It should not be inferred that, because TOUGH and Garg's solution disagree in the first ten seconds of the simulation, that the TOUGH solution is incorrect, since Garg's solution is also numerical. In fact, the small discrepancies could be attributed to numerical errors. TOUGH also predicted 


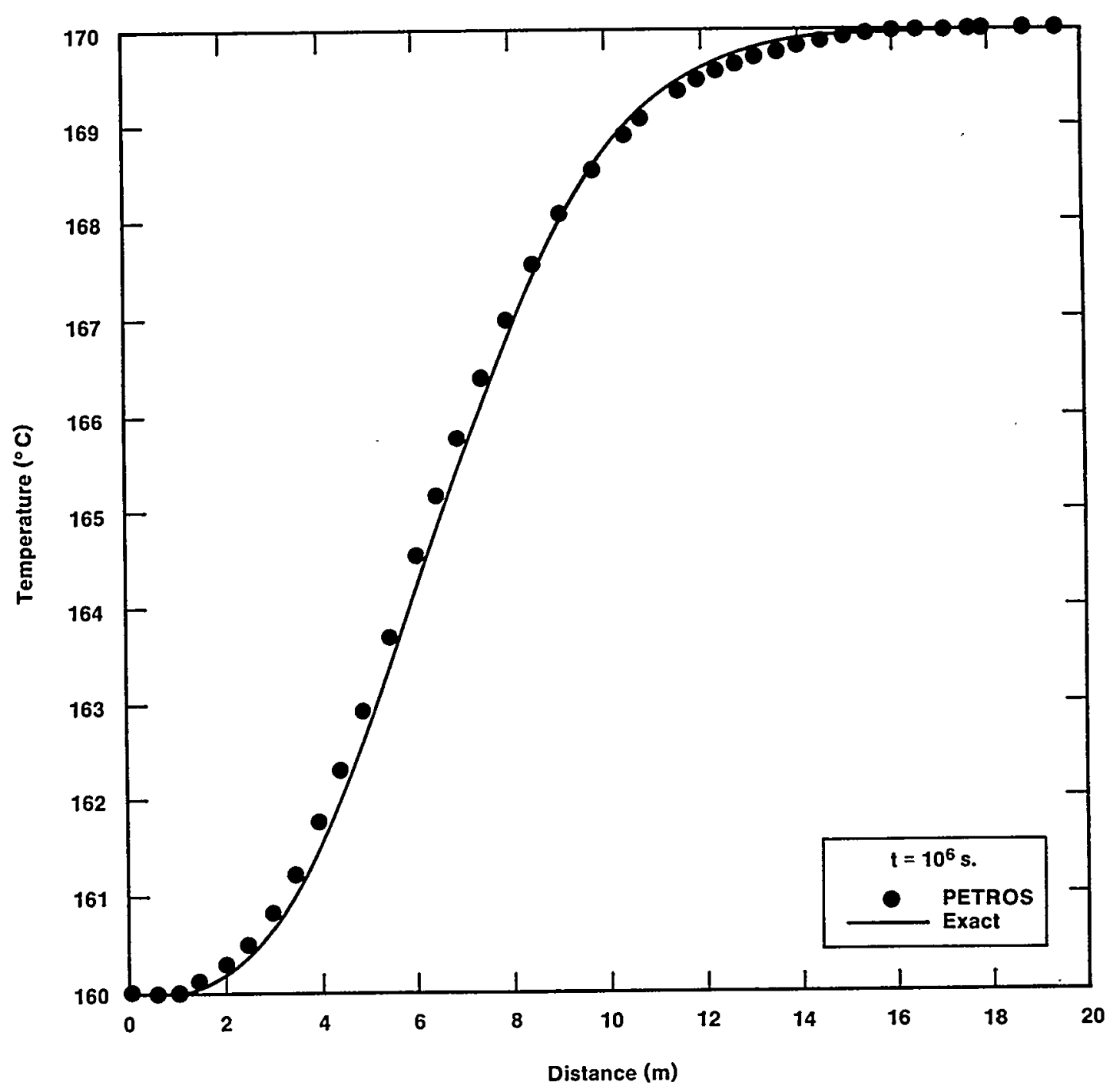

Figure 4.18: Comparison of PETROS Results with Exact Solution at $1 \times 10^{6} \mathrm{~s}$ for the Radial Heat Transport Problem 


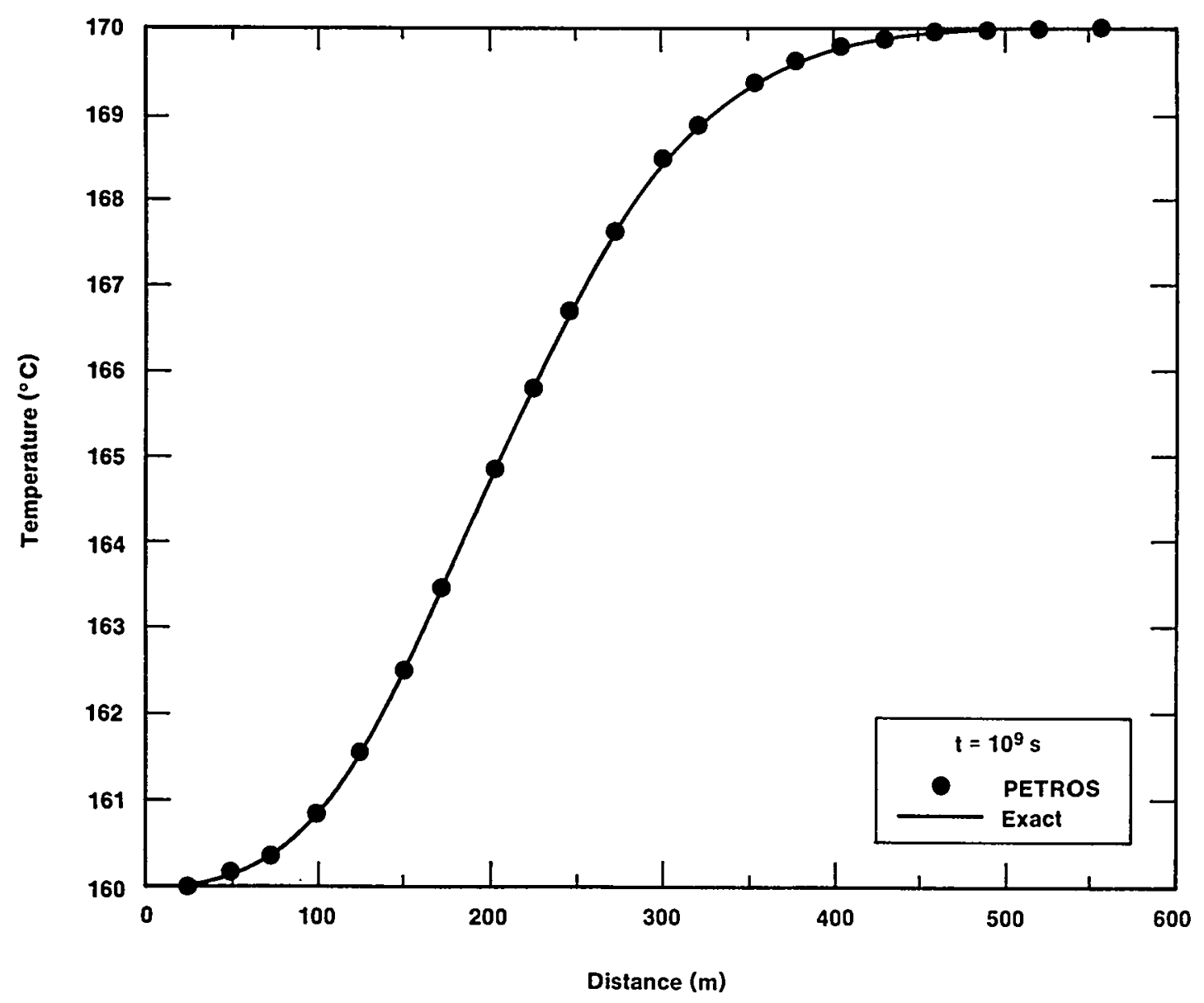

Figure 4.19: Comparison of PETROS Results with Exact Solution at $1 \times 10^{9} \mathrm{~s}$ for the Radial Heat Transport Problem 


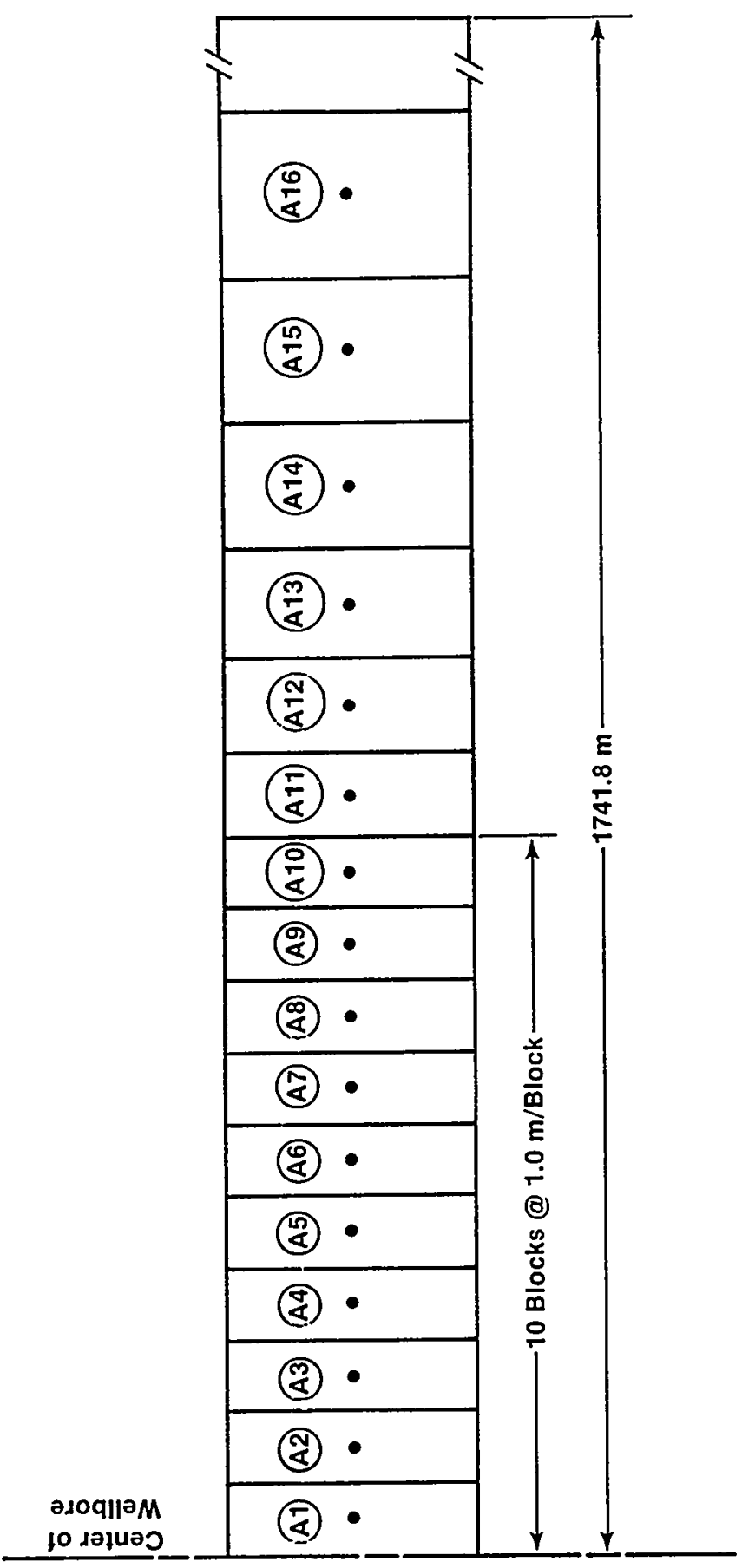

章 


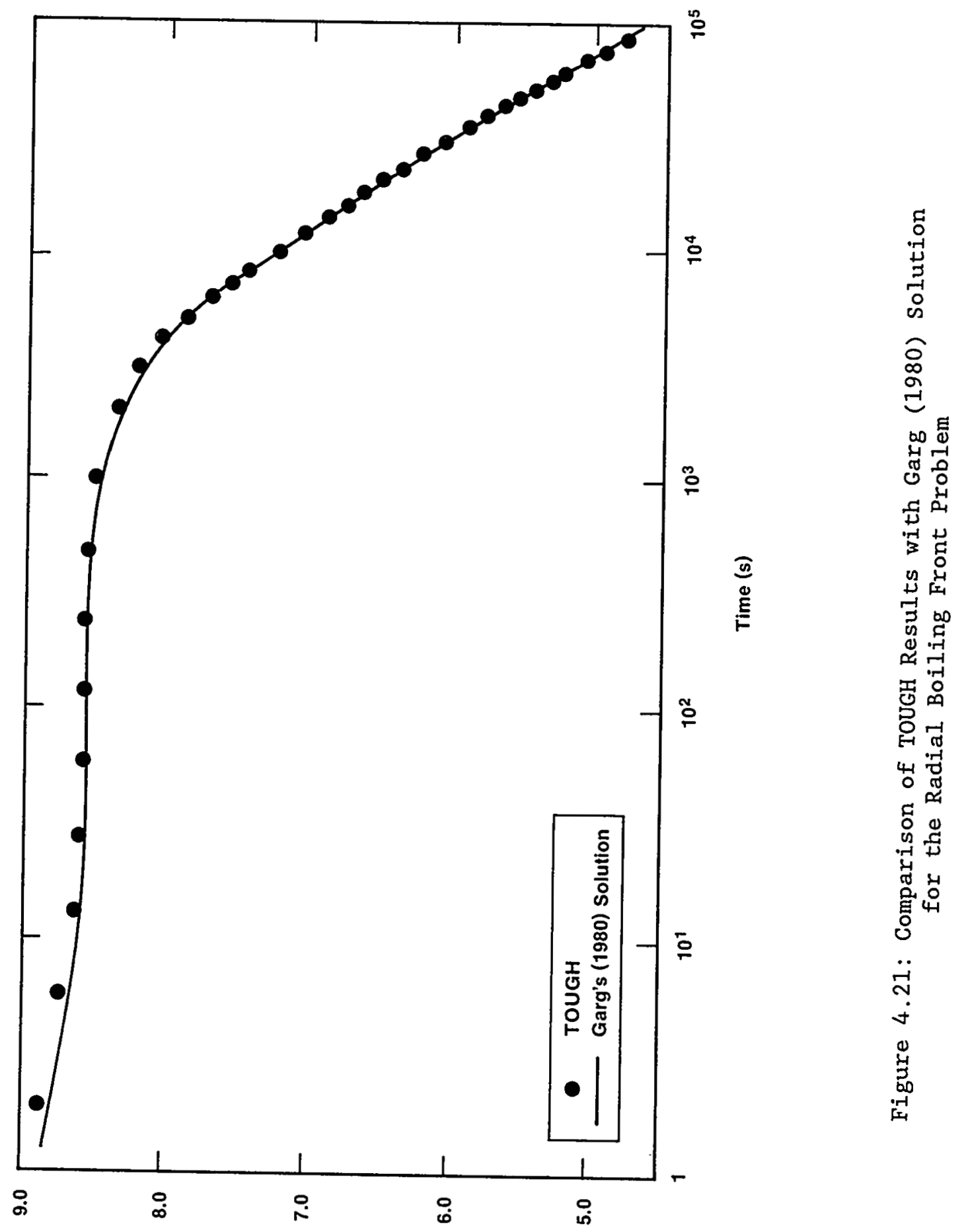

(EdW) כגnssedd әגOQ\|⿵M 
the late time straight line pressure distribution as indicated by Garg's asymptotic solution. Overall, there is an excellent agreement between the TOUGH results and Garg's numerical solution.

\subsubsection{NORIA}

The NORIA code could not successfully simulate the radial boiling front problem. It suffered from several problems. First, it required a very small time-step size in order to start the simulation. Second, as time increased, the time step also increased but reached a maximum size which varied between. about 0.05 and $0.3 \mathrm{~s}$. These small time steps produced slow progress in time. Third, the time-step size then began to decrease as time progressed until it became smaller than the initial time-step size, which caused NORIA to stop running.

\subsubsection{PETROS}

The PETROS code produced a solution to the radial bolling front problem. Unfortunately, the solution did not compare favorably with Garg's (1980) solution, either qualitatively or quantitatively.

The grid used in the PETROS simulation is presented in Figure 4.22. It consisted of 51 nodes. The first 10 nodes were each spaced $1.0 \mathrm{~m}$ apart. The first node is located $1.0 \mathrm{~m}$ from the center of the wellbore. For subsequent nodes, the ratio of the distance between a pair of nodes is approximately 1.14907 of the distance between the preceding pair of nodes. This ratio is the same as that used in the TOUGH simulation of this problem. The distance between the center of the wellbore and the outer boundary is $2000.0 \mathrm{~m}$.

At the wellbore, the mass flux into the well consisted of both liquid and vapor. The mass flux of each phase depended on its mobility ratio. A zero heat flux was assigned at the wellbore. Zero mass flux conditions and a fixed temperature of $300^{\circ} \mathrm{C}$ were assigned at the outer boundary. The initial temperature and saturation were assigned as $300^{\circ} \mathrm{C}$ and 0.9999 , respectively. Air was not included in the simulation of this problem. Because the vapor equation does not contain a transient term, no initial condition was required for it. PETROS calculated an initial constant vapor pressure prior to the simulation of the problem.

The results of the PETROS simulation are presented in Figure 4.23. There is a poor comparison between the PETROS solution and the Garg (1980) solution. PETROS assigned an initial constant pressure of approximately $8.5 \mathrm{MPa}$, which is the equilibrium pressure for a temperature of $300^{\circ} \mathrm{C}$. This pressure contrasts with the $9.0 \mathrm{MPa}$ specified in the problem description. The difference between the two pressures affected the wellbore pressure. On the initial time step, it dropped to approximately $8.1 \mathrm{MPa}$. As a result, PETROS predicted vaporization immediately after the start of the simulation, while in Garg's solution vaporization did not occur until ten seconds into the simulation. The net result of this is a very rapid pressure drop in the wellbore. 


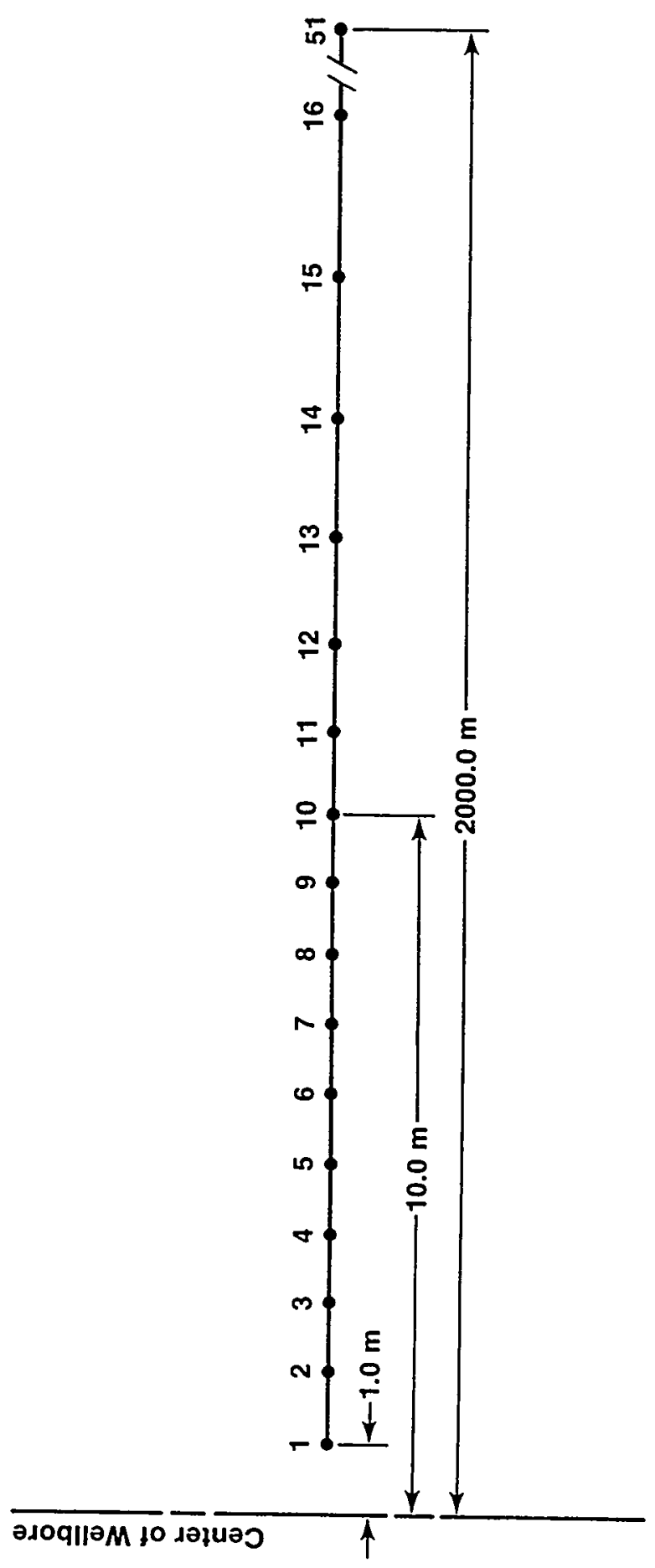

Oت 


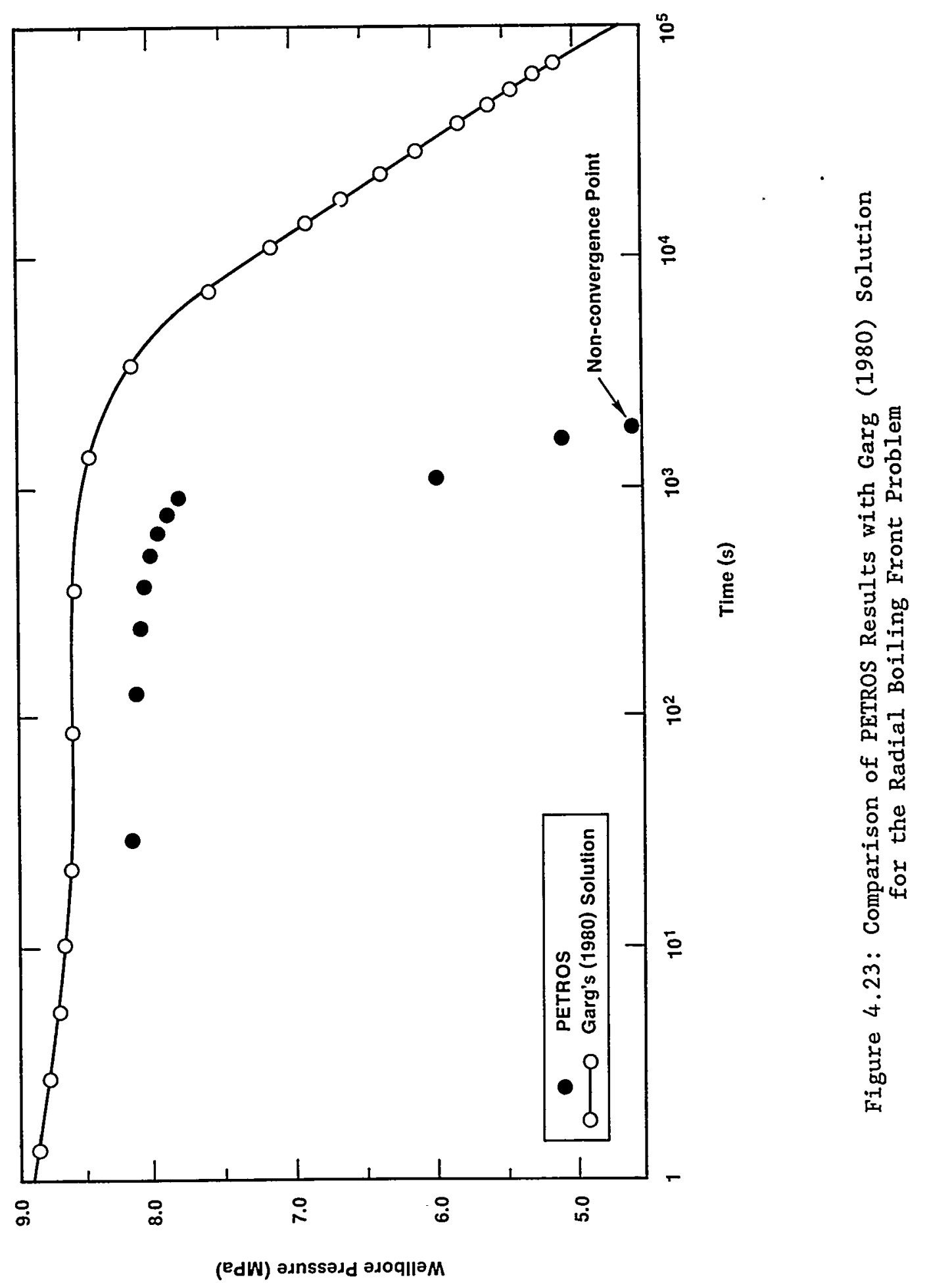

$-71-$ 
It appears, though, that the simulation is poor for another reason. The saturation at the wellbore node and at nodes near the wellbore exhibited a rather strange behavior. For most of the simulation, the saturation at the wellbore stays at 1.0 and, at the adjacent node, the saturation decreases with time. One would expect saturation to be less in the wellbore node than at the adjacent node. The reason for this behavior is not known but it was speculated that PETROS could not handle the mass flux boundary condition at the wellbore boundary.

\subsubsection{Heat Pipe}

\subsubsection{TOUGH}

TOUGH successfully simulated the heat pipe problem in $128.5 \mathrm{~s}$ of CPU time. The grid, consisting of 91 grid blocks, used to model the problem is presented in Figure 4.24. The first grid block, LB00, was assigned a large volume and was used to control the boundary conditions at the left side of the modeled region. The remaining 90 grid blocks, SAM00 to SAM89, were each $0.025 \mathrm{~m}$ wide.

The left boundary was held fixed at an ambient air pressure of $101330 \mathrm{~Pa}$, a saturation of 0.5 , and a temperature of $70^{\circ} \mathrm{C}$. The right boundary conditions were chosen as zero flux conditions for both mass and heat. The flux of heat into the right side of the modeled region was handled by injecting $100 \mathrm{~W}$ into the center of the SAM89 grid block. Initial conditions for pressure, temperature, and saturation were set at $101330 \mathrm{~Pa}, 70^{\circ} \mathrm{C}$, and 0.50 , respectively. Since the required solution was at steady state, the values of the initial conditions should be irrelevant, but because TOUGH reaches the steady-state solution via a transient solution, initial conditions had to be specified.

A comparison of the TOUGH-generated solution with the fourth-order Runge-Kutta solution of Udell and Fitch (1985) is presented in Figure 4.25. Because the liquid and gas properties were pressure- and temperaturedependent in TOUGH and constants in the Udell and Fitch model, we should have expected some quantitative differences in the two solutions. However, both solutions should exhibit, at least, a similar qualitative behavior of the dependent variables. Figure 4.25 shows an excellent agreement between the two solutions for temperature and the mole fraction of air in the gas.

However, the saturation results for the two solutions differ. TOUGH produced the higher saturations, but both solutions are qualitatively similar. The saturation results were probably strongly affected by the temperature and pressure dependence of the liquid and gas properties. Overall, there appears to be a good agreement between the TOUGH results and the results of Udeli and Fitch (1985).

\subsubsection{NORIA}

NORIA could not simulate the heat pipe problem with $1 \mathrm{~h}$ of CPU time. It had a similar problem with time-step size as mentioned in Section 4.1.4.2. The 


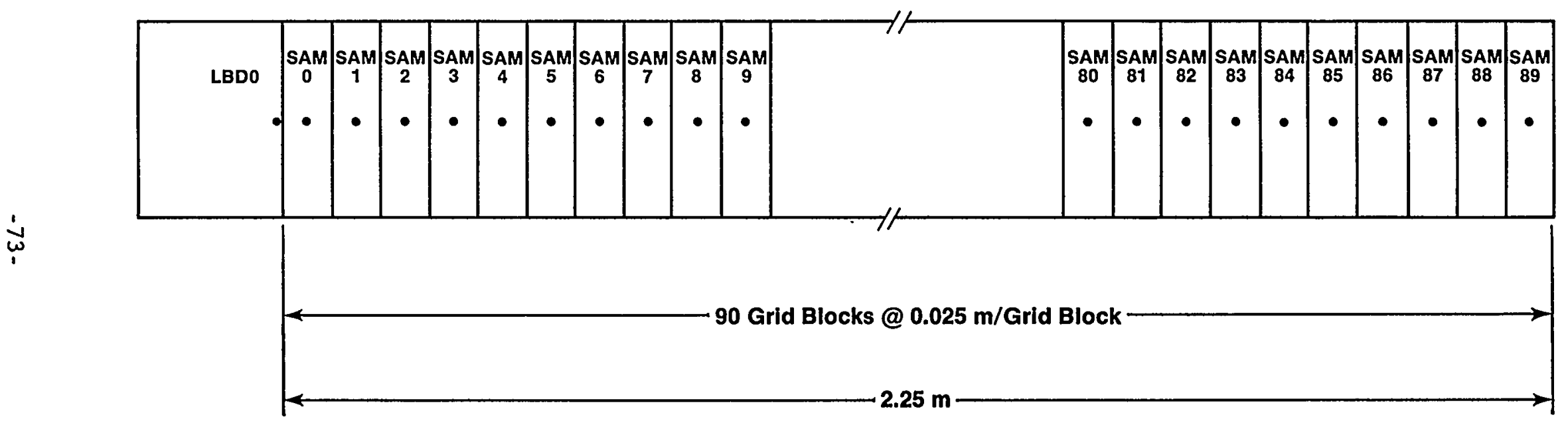

Figure 4.24: TOUGH Grid for the Heat Pipe Problem 


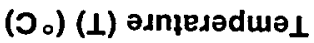

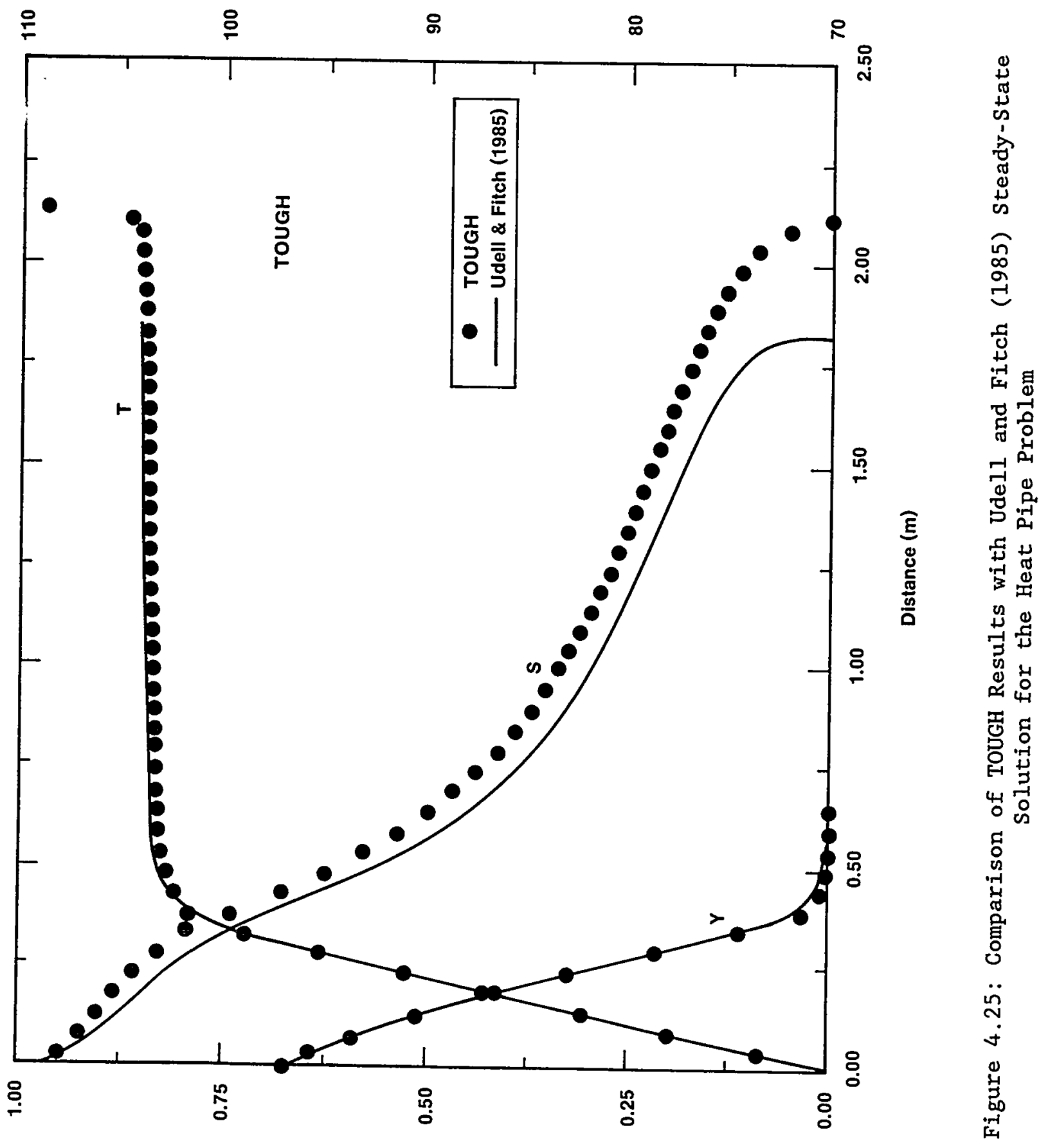

(A) UO!ฺOEd] 이

(S) uo!̣ednites 
time step was initialized at $1.0 \mathrm{~s}$ and was gradually increased until a time of approximately $1.0 \times 10^{5} \mathrm{~s}$. At this time, the time-step size started to slowly oscillate reaching values as small as $85 \mathrm{~s}$ and as large as $6836 \mathrm{~s}$. This behavior continued until a time of approximately $504000 \mathrm{~s}$. At this time, the time-step size began to rapidly decrease between time steps until it became less than the initial time-step size, at which time the simulation stopped.

The grid used to solve the problem is presented in Figure 4.26. The grid consisted of 45 elements, each $0.05 \mathrm{~m}$ wide. The liquid pressure, vapor partial pressure, air partial pressure and temperature on the left boundary were held fixed at $58056.23 \mathrm{~Pa}, 10131.23 \mathrm{~Pa}, 91198.77 \mathrm{~Pa}$, and $70^{\circ} \mathrm{C}$, respectively, These conditions corresponded to a saturation of 0.5 and a gas density of $0.960 \mathrm{~kg} / \mathrm{m}^{3}$, which was specified in the problem description. The boundary conditions at the right side were specified as zero flux for the liquid, vapor, and air flow, and $100 \mathrm{~W} / \mathrm{m}^{2}$ directed inward for the heat flow. The initial conditions are the same as the left side boundary conditions.

Although NORIA did not run long enough to allow a direct quantitative comparison with the Udell and Fitch (1985) solution, a qualitative comparison was possible between the transient NORIA solution and the steady-state solution of Udell and Fitch. On the right side of the modeled region, the soil began to dry out just as predicted by Udell and Fitch. However, in that region NORIA predicted a temperature in excess of $120^{\circ} \mathrm{C}$, while Udell and Fitch predicted a temperature near $104^{\circ} \mathrm{C}$. It is expected that, if NORIA could have generated a solution, it would have differed from the Udell and Fitch solution.

\subsubsection{PETROS}

PETROS could not generate a steady-state solution. It could only generate a solution up to approximately $250000 \mathrm{~s}$ and then only by excluding air from the simulation. The only reason times greater than $250000 \mathrm{~s}$ could not be simulated was that for large times, time steps became very small, less than one second, and the solution advanced very slowly in time. The problem was also simulated including air. However, as the partial pressure of air became very small, $0.001 \mathrm{~Pa}$, the air partial pressure solution converged slowly. Eventually, the solution did not converge at all.

The grid used to simulate the problem is presented in Figure 4.27. It consisted of 91 nodes spaced $0.025 \mathrm{~m}$ apart. For the case without air, the left boundary was held fixed at a saturation of 0.5 , a temperature of $70^{\circ}$ $\mathrm{C}$, and a vapor partial pressure of $101330 \mathrm{~Pa}$. The right side was impermeable to liquid and gas flow and had a heat flux of $100 \mathrm{~W} / \mathrm{m}^{2}$ directed inward. Initial conditions were the same values as the left side boundary conditions. For the case including air, the left side saturation, temperature, vapor partial pressure, and air partial pressure were held fixed at $1.0,70^{\circ} \mathrm{C}, 10131.23 \mathrm{~Pa}$, and $91198.77 \mathrm{~Pa}$, respectively. The right-side boundary conditions were the same as in the no-air case and the initial conditions were the same as the left-side boundary conditions. 


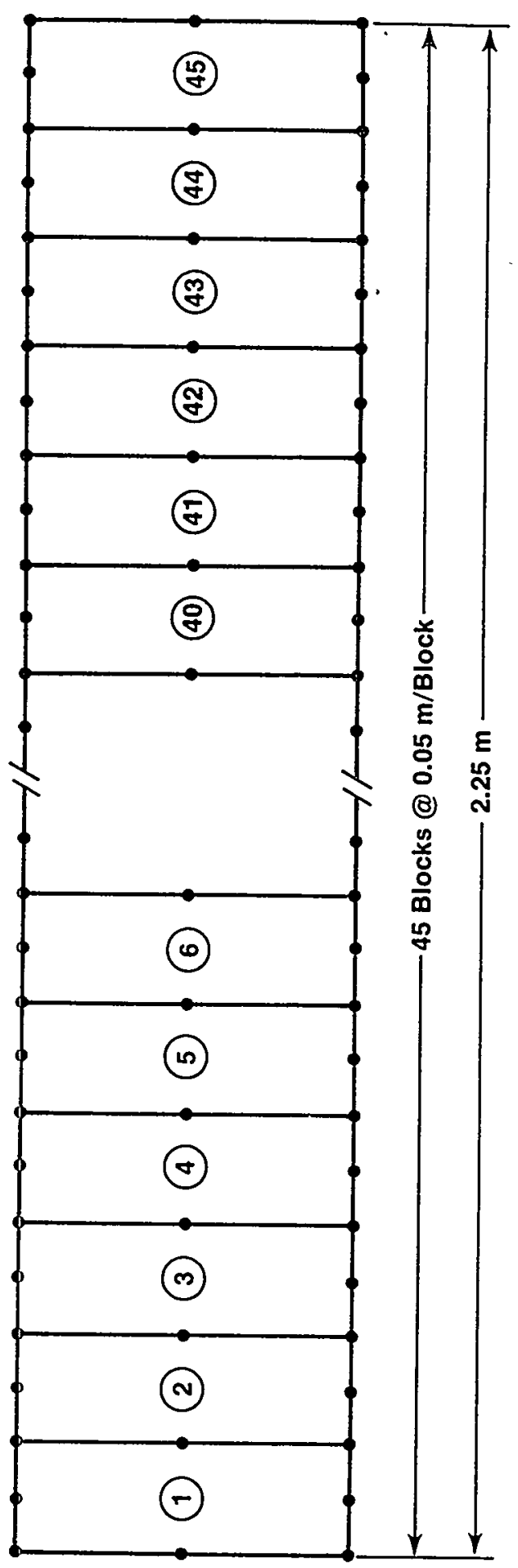

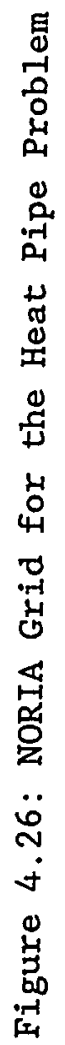




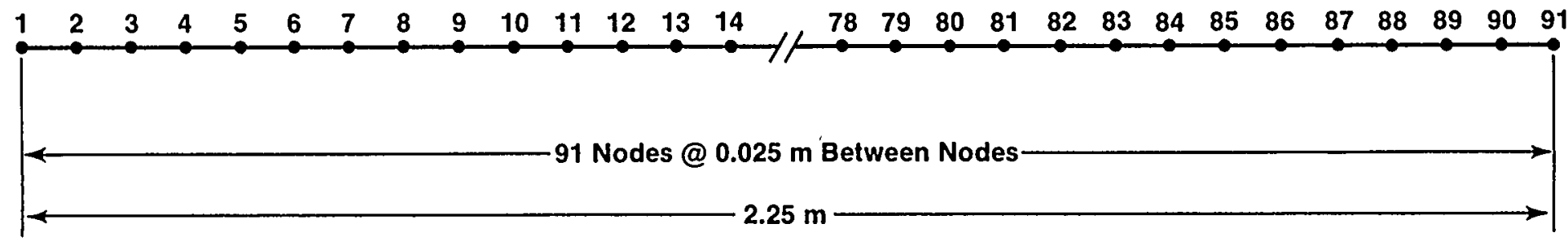

Figure 4.27: PETROS Grid for the Heat Pipe Problem 


\subsection{Comparison With Experimental Results}

\subsubsection{Two-Dimensional Infiltration}

\subsubsection{TOUGH}

Approximately $6300 \mathrm{~s}$ (one and three-quarter hours) of the $8-\mathrm{h}$, two-dimensional infiltration experiment could be simulated with TOUGH. After the $6300 \mathrm{~s}$ of simulation time, time steps became very small and the solution progressed slowly. The first $6300 \mathrm{~s}$ of simulation time required 39 time steps and the next $422 \mathrm{~s}$ required 231 time steps. This gives an idea of the CPU time consumed by the small time steps. The simulation was stopped after $1 \mathrm{~h}$ of CPU time.

The grid used to solve the problem is presented in Figure 4.28. It was 13 grid blocks wide and 5 grid blocks high. In the horizontal direction, grid block columns 1 through 12 were $0.25 \mathrm{~m}$ wide. This width was chosen to force the $0.50-\mathrm{m}$-wide infiltration source to cover exactly two grid blocks. Grid block column 13 was used to control the constant pressure boundary condition on the right side of the modeled region. In the vertical direction, grid blocks rows $A, B, C$, and D were each $0.1625 \mathrm{~m}$ high and were located in the saturated zone. Grid blocks rows $\mathrm{E}$ through $\mathrm{N}$ were each $0.1350 \mathrm{~m}$ high and were located in the unsaturated zone. In the top grid block row, grid blocks 01 and 02 were extremely thin and were used to control the infiltration rate boundary condition. Grid blocks 03 through 012 were very large and were used to set a the zero infiltration rate boundary condition along the top of the grid.

The lack of any boundary grid blocks along the bottom and left side of the grid implies zero mass and heat fluxes out these two sides. Along the right side, grid blocks A13, B13, C13, and D13 were held fixed at a saturation of 1.0 , a temperature of $20^{\circ} \mathrm{C}$, and a hydrostatic pressure distribution, which ranged between an atmospheric pressure of $101330 \mathrm{~Pa}$ at the water table and $107694 \mathrm{~Pa}$ at the bottom boundary. Grid blocks E13, F13, G13, H13, I13, $\mathrm{J} 13, \mathrm{~K} 13, \mathrm{L13}, \mathrm{M} 13$, and $\mathrm{N} 13$ were held fixed at a temperature of $20^{\circ} \mathrm{C}$, a gas pressure of $101330 \mathrm{~Pa}$ (one atmosphere), and a saturation that implied zero vertical flux of liquid. Actually, a seepage-face boundary condition should be applied to the right-side grid blocks above the water table, but TOUGH does not have this capability. Grid blocks 01 and 02 were each assigned a liquid mass input rate of $0.0103 \mathrm{~kg} / \mathrm{s}$, which corresponds to an input rate of $4.1111 \times 10^{-5} \mathrm{~m} / \mathrm{s}$. Because there were no grid blocks on top of the 01 and 02 grid blocks to allow the escape of mass or heat, zero mass and zero heat flux conditions were assumed at the top of these grid blocks. Grid blocks 03 through 012 were assigned zero liquid saturation. This prevented the flow of liquid out of the top of the grid, and approximated the seepage-face boundary conditions as long as the liquid saturation was less than full saturation at the top boundary. Because of the large volumes assigned to grid blocks 03 through 012 , the temperature and gas pressure were held fixed at $20^{\circ} \mathrm{C}$ and $101330 \mathrm{~Pa}$, respectively. For initial conditions grid block rows $A, B, C$, and $D$ in the saturated zone were assigned a saturation of 1.0 , a temperature of $20^{\circ} \mathrm{C}$, and the hydrostatic pressure distribution as described above. Grid block rows E, F, G, H, I, J, 


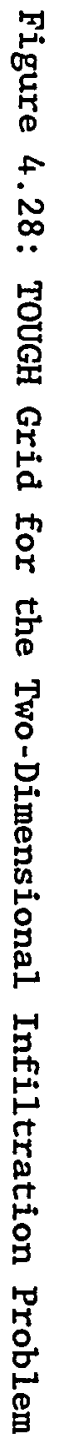

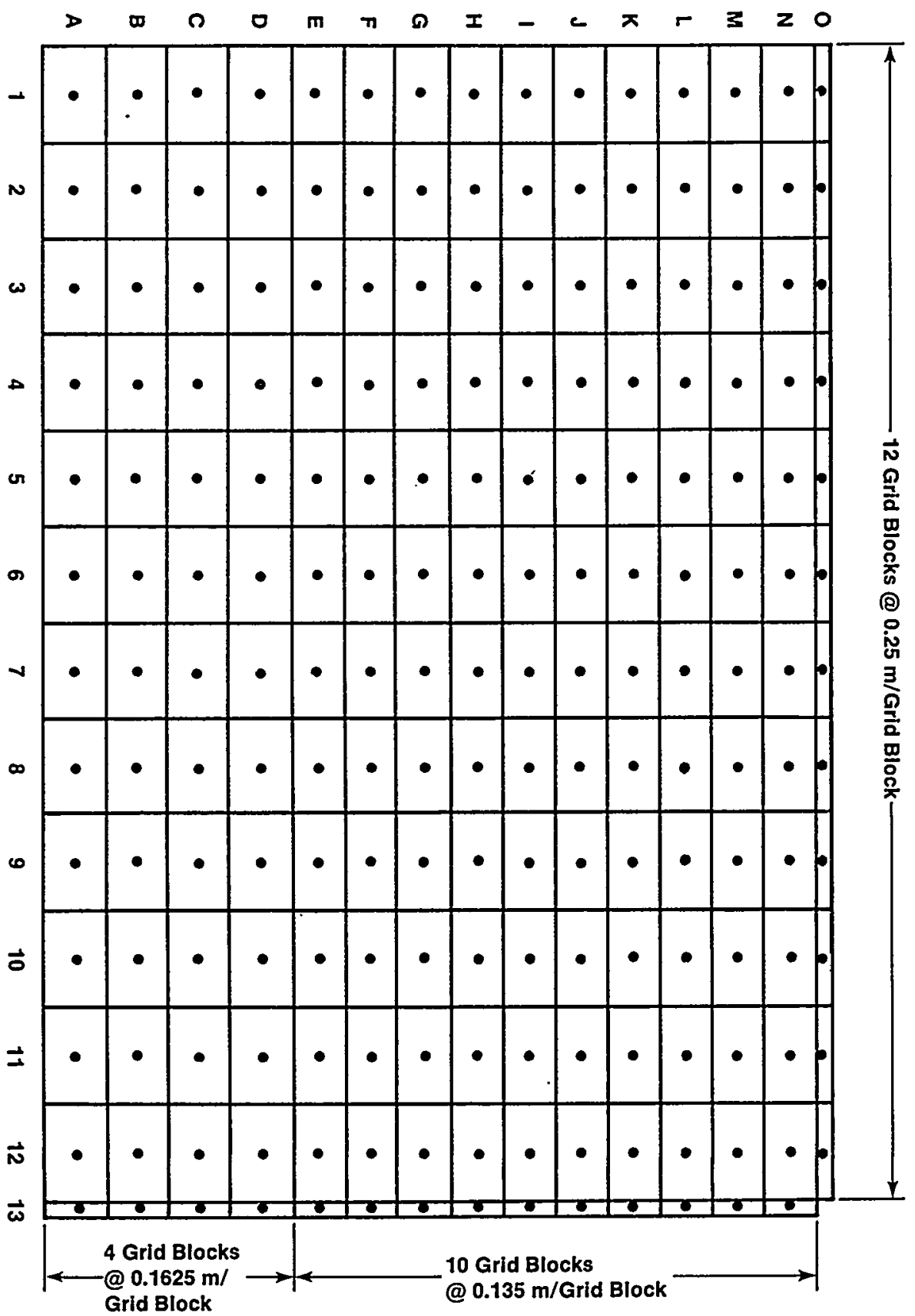


$K, L, M, N$, and 0 in the unsaturated zone were assigned a gas pressure of $101330 \mathrm{~Pa}$, a temperature of $20^{\circ} \mathrm{C}$, and saturation values which correspond to zero liquid flux in the vertical direction.

A comparison of the predictions from TOUGH with some of the experimental results is presented in Figure 4.29. For $x=0.19$, the comparison at $t=$ $0.5,1.0$, and $1.5 \mathrm{~h}$ is fairly good. At $t-1.0$ and $1.5 \mathrm{~h}$, a small

discrepancy between the TOUGH solution and the experimental results exists at a depth of approximately 0.40 to $0.50 \mathrm{~m}$. This appears to be due to a layered heterogeneity in the experiment because the same type of behavior is seen in the experimental curve for $x=0.49 \mathrm{~m}$.

For $\mathrm{x}=0.49 \mathrm{~m}$, there is poor agreement between TOUGH and the experimental results. It is suspected that the poor agreement is due to two factors. First, $x=0.49 \mathrm{~m}$ is very near the point on the top boundary of the experiment where the application of water stops and the seepage face begins. Under this point, especially at small times, a steep saturation front forms in the horizontal direction. In order to better simulate this front, it would have been necessary to use a finer grid spacing in this region. Second, the large discrepancy is partially due to the interpolation scheme used to calculate the saturation at $x=0.49 \mathrm{~m}$. The scheme is based on saturation values at $\mathrm{x}=0.375 \mathrm{~m}$, directly under the recharge region, and $\mathrm{x}=$ $0.625 \mathrm{~m}$, a nearly dry region. The interpolation scheme does not take into account the actual behavior of the saturation front between the two points but approximates it with a linear function.

For $x=0.79 \mathrm{~m}$ at $t=1.5 \mathrm{~h}$, there appears to be a good qualitative agreement between TOUGH and the experimental results in that the shape of the two curves is similar. In a quantitative sense, TOUGH predicts water moving out faster to the right than what is actually observed. In order to be sure if this is the case, the code results would have to be compared with the experimental results for larger times.

\subsubsection{NORIA}

The grid used to simulate the two-dimensional infiltration experiment with NORIA is presented in Figure 4.30. It consisted of 12 elements in the horizontal direction and 10 elements in the vertical direction for a total of 120 elements and 405 nodes. Elements 1 through 36, all located in the saturated zone, were each $0.2167 \mathrm{~m}$ high and $0.25 \mathrm{~m}$ wide. Elements 37 through 48 , each $0.15 \mathrm{~m}$ high and $0.25 \mathrm{~m}$ wide, were located in the unsaturated zone and served as transition elements to the overlying elements. Elements 49 through 120, located in the unsaturated zone, were each $0.20 \mathrm{~m}$ high and 0.25 m wide.

Boundary conditions along the left side and bottom of the grid were all assigned to reflect zero fluxes of water, air, vapor, and heat. Elements 12 , 24 , and 36 on the right side were assigned effective pressures of 107693.8 $\mathrm{Pa}$ to reflect the depth of water in the saturated zone and the atmospheric pressure. Unsaturated zone elements along the right side of the grid were assigned a zero liquid flux condition to reflect a seepage-face boundary. For all elements along the right side of the grid, an air partial pressure of $100000 \mathrm{~Pa}$, a vapor partial pressure of $11330 \mathrm{~Pa}$, and a temperature of 


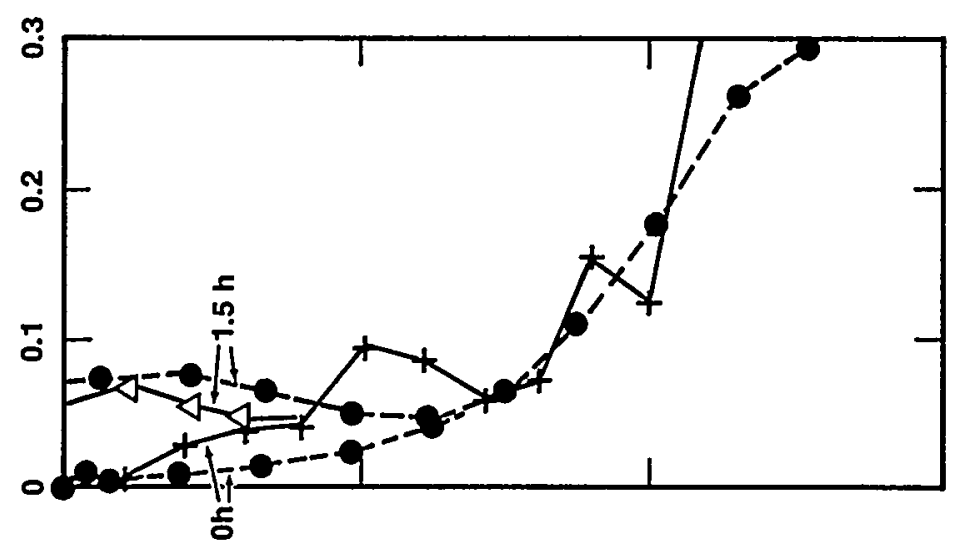

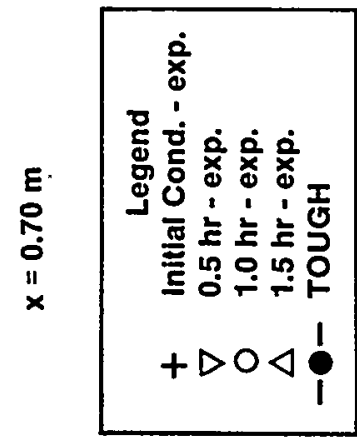

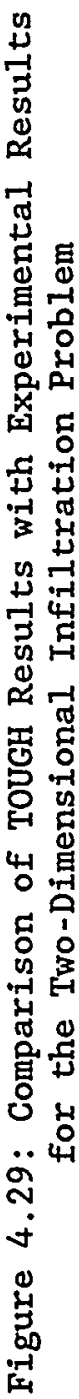

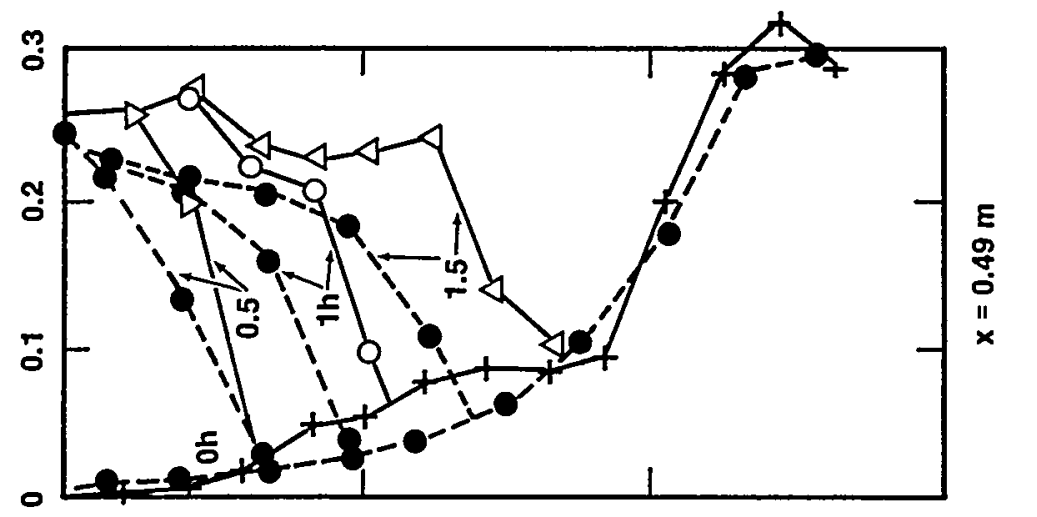

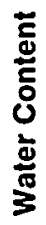

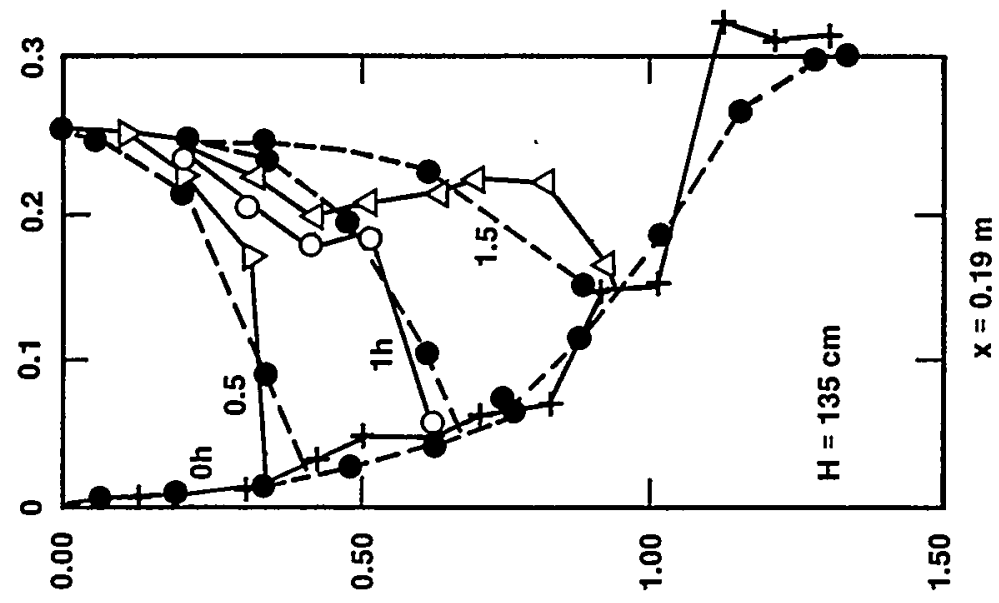

(u) $47 \mathrm{~d} ә$ 


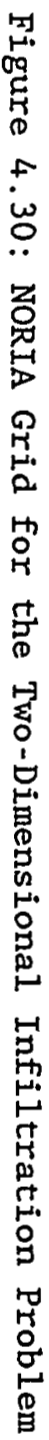

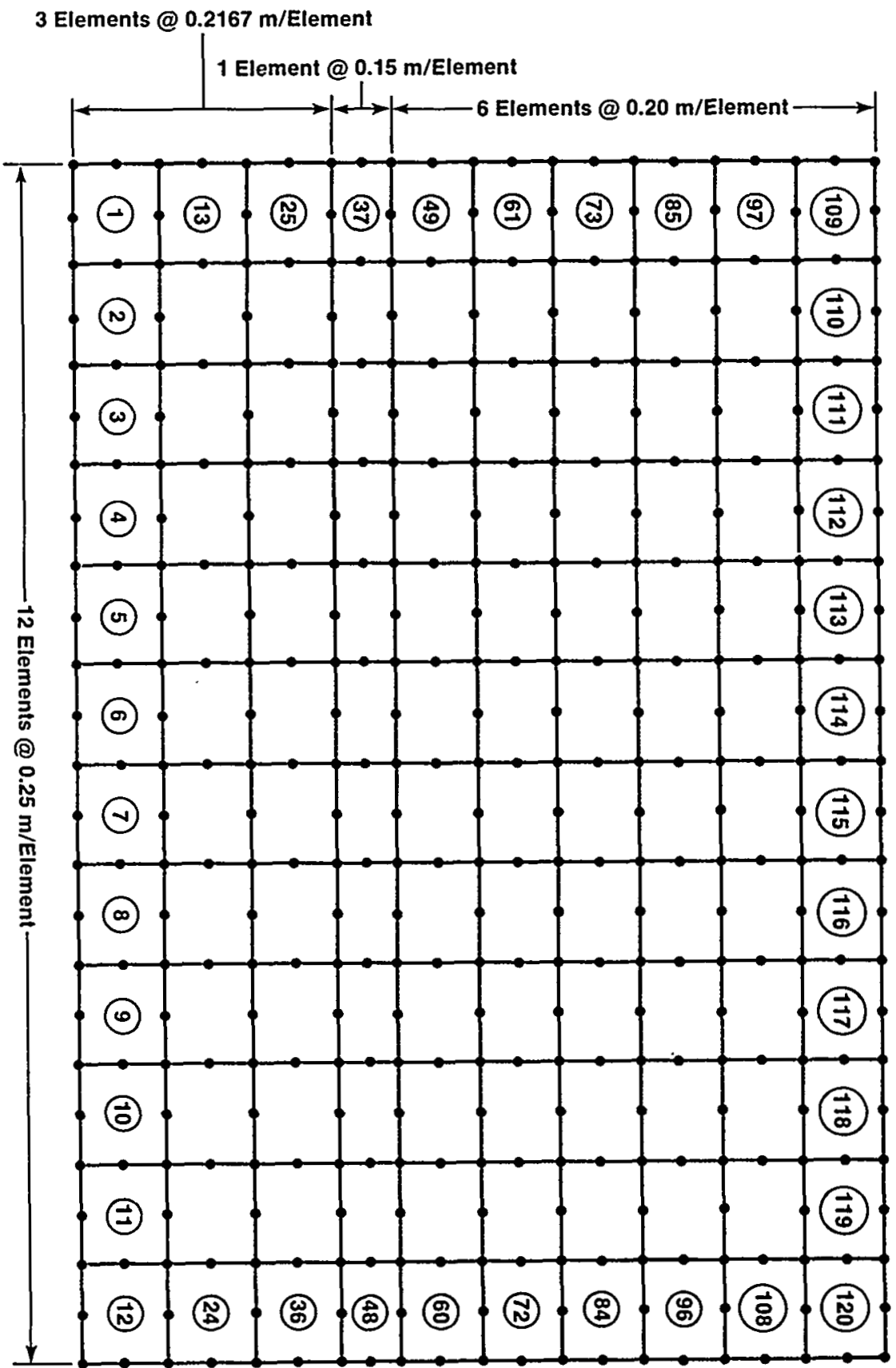


$20^{\circ} \mathrm{C}$ were assigned. Along the top of elements 109 and 110 a flow rate of $4.11 \times 10^{-5} \mathrm{~m} / \mathrm{s}$ directed inward was prescribed to simulate the recharge zone. The remaining elements along the top of the grids were assigned a zero liquid flux to reflect a seepage face. In addition, all elements along the top of the grid were assigned boundary conditions of $100000 \mathrm{~Pa}$ for air partial pressure, $11330 \mathrm{~Pa}$ for vapor partial pressure, and $20^{\circ} \mathrm{C}$ for temperature.

NORIA could not simulate this problem. It ran for approximately $8.5 \mathrm{~s}$ and then stopped because the time-step size became too small. The initial time step was $1.0 \mathrm{~s}$ and it increased with each time step to approximately $4.8 \mathrm{~s}$ at which time it started to get smaller. It appears that the vapor partial pressure term was the main reason causing the time steps to decrease. Thedifference between the predictor and corrector vapor partial pressure solution is so large that time-step size must be reduced in order to meet NORIA's time-step-size criteria.

\subsubsection{PETROS}

PETROS, being a one-dimensional code, was not capable of solving the two-dimensional infiltration problem.

\subsubsection{Convection CeII}

\subsubsection{TOUGH}

The integrated finite-difference grid used in the simulation of the convection-cell problem with TOUGH is presented in Figure 4.31. The grid consisted of 462 grid blocks, of which 62 were used to control boundary conditions.

Grid blocks T0 to EEO, representing the heater, controlled the flux of thermal energy into the system. Grid blocks 001 through 0010 controlled the constant temperature and pressure conditions at the top of the convection cell. Grid blocks All through NNII, assigned very small volumes and very large heat capacities, were used to control the zero mass flux and constant temperature boundary conditions.

Along the left side and bottom of the grid, zero mass flux and zero heat flux boundary conditions were assigned. These conditions implied that no boundary grid blocks were necessary there. At the top of the grid, the large volume grid blocks, 001 through 0010 , were assigned a fixed temperature of $21.5^{\circ} \mathrm{C}$ and a fixed pressure of $200000 \mathrm{~Pa}$. The extremely large volumes of these grid blocks allowed only very small changes to occur within the grid blocks such that the pressures and temperatures essentially remained constant. Along the right side, the small volume grid blocks, All through 0011, with large heat capacities handled two types of boundary conditions. The small volume assigned to these grid blocks forced the right edge of these grid blocks almost up against the right edge of grid blocks Al0 through 0010. This, in effect, forced the zero mass flux boundary at the right side of grid blocks AlO through 0010. On the other hand, the large heat 


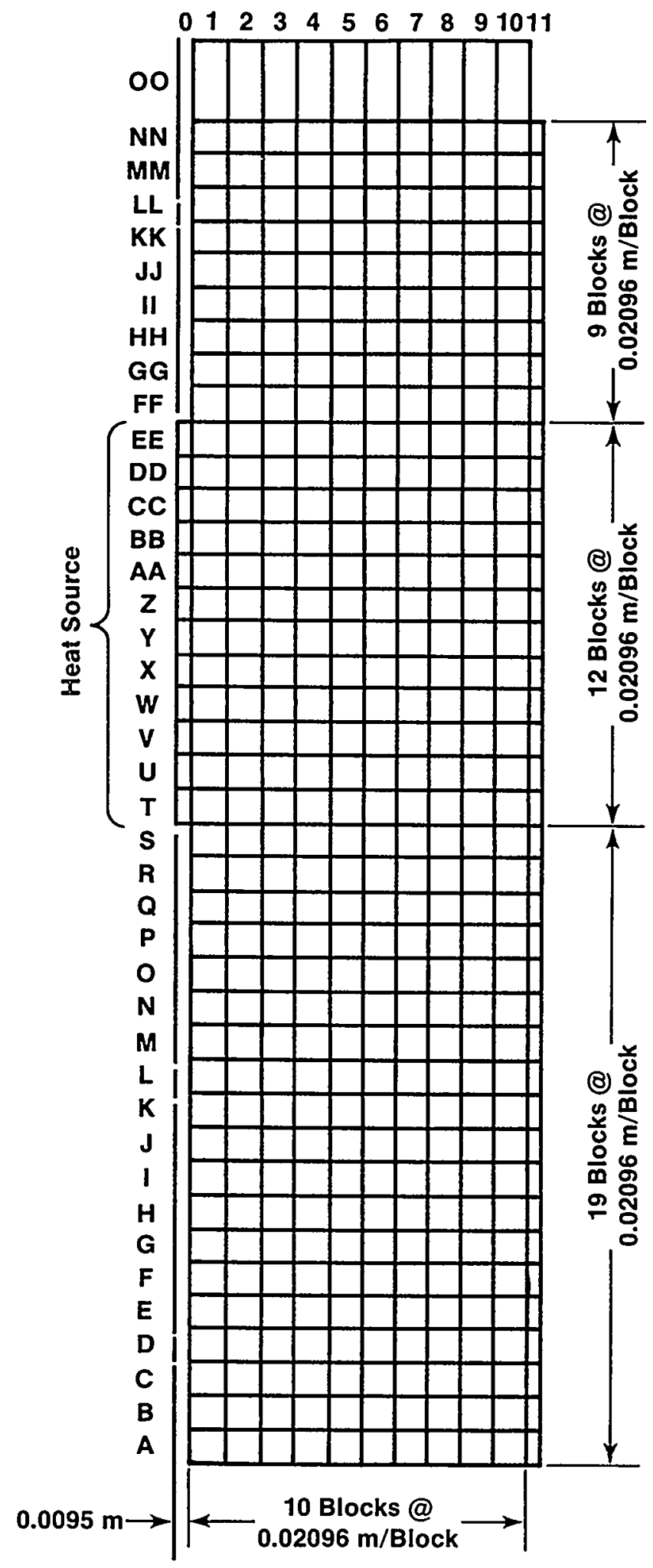

Figure 4.31: TOUGH Grid for the Convection Cell Problem 
capacities assigned to the grid blocks A11 through 0011 kept the temperature changes in these grid blocks very small, essentially keeping the temperature fixed. The initial conditions assigned to the grid blocks included a temperature of $21.5^{\circ} \mathrm{C}$ and a hydrostatic pressure distribution based on a pressure of $200000 \mathrm{~Pa}$ assigned at the interface of the NN-00 row of grid blocks.

TOUGH could not simulate the convection cell behavior of the experiment. The results of the TOUGH simulation are presented in Figure 4.32. TOUGH's predicted temperatures were higher than the experimental ones by $15^{\circ} \mathrm{C}$ for small times and as much as $30^{\circ} \mathrm{C}$ for large times. In addition, temperatures at the top and bottom of the rods differed by $30^{\circ} \mathrm{C}$ for the experiment, while TOUGH predicted a difference of less than $10^{\circ} \mathrm{C}$.

\subsubsection{NORIA}

The finite-element grid used with NORIA to simulate the convection cell problem is presented in Figure 4.33. The grid consisted of 21 elements in the vertical direction and 5 elements in the horizontal direction for a total of 105 elements and 368 nodes. The width and height of all elements except those in the top and bottom rows were $0.04192 \mathrm{~m}$. The height of elements in the top and bottom rows was one-half the height of the other elements, or $0.02096 \mathrm{~m}$. The reason for the one-half height elements on the top and bottom rows was to align the elements with the heater as shown in Figure 4.33 .

Along the bottom boundary, zero flux conditions for liquid, vapor, air, and heat, were assigned. Along the outside boundary, the temperature was held fixed at $21.5^{\circ} \mathrm{C}$, while the liquid, vapor, and air were assigned zero-flux conditions. At the top boundary, the temperature was held fixed at $21.5^{\circ} \mathrm{C}$, the effective liquid pressure at $102168.4 \mathrm{~Pa}$ (atmospheric pressure plus the height of water in the convection cell), vapor pressure at $11330 \mathrm{~Pa}$ and air pressure at $90000 \mathrm{~Pa}$. Along the inner boundary, zero flux conditions were assigned for the liquid, air, and vapor. A zero heat flux condition was assigned everywhere along the inner boundary except along the heater where the heat flux was $278.3 \mathrm{~W} / \mathrm{m}$. The initial conditions for effective liquid pressure, vapor partial pressure, air partial pressure, and temperatures were assigned values of $102168.4 \mathrm{~Pa}, 11330 \mathrm{~Pa}, 90000 \mathrm{~Pa}$, and $21.5^{\circ} \mathrm{C}$, respectively.

NORIA could not simulate the convection cell problem. As in other problems, NORIA ran for several time steps, then started reducing its time-step size until it became smaller than its initial value, at which time the simulation terminated. The cause of the time-step problem is not clear but it appears to be due to large differences between the predictor and corrector vapor partial pressure solutions. NORIA uses differences between the predictor and corrector solutions when estimating a new time-step size.

\subsubsection{PETROS}

PETROS, being a one-dimensional code, was not capable of solving the convection cell problem, which was a two-dimensional problem. 


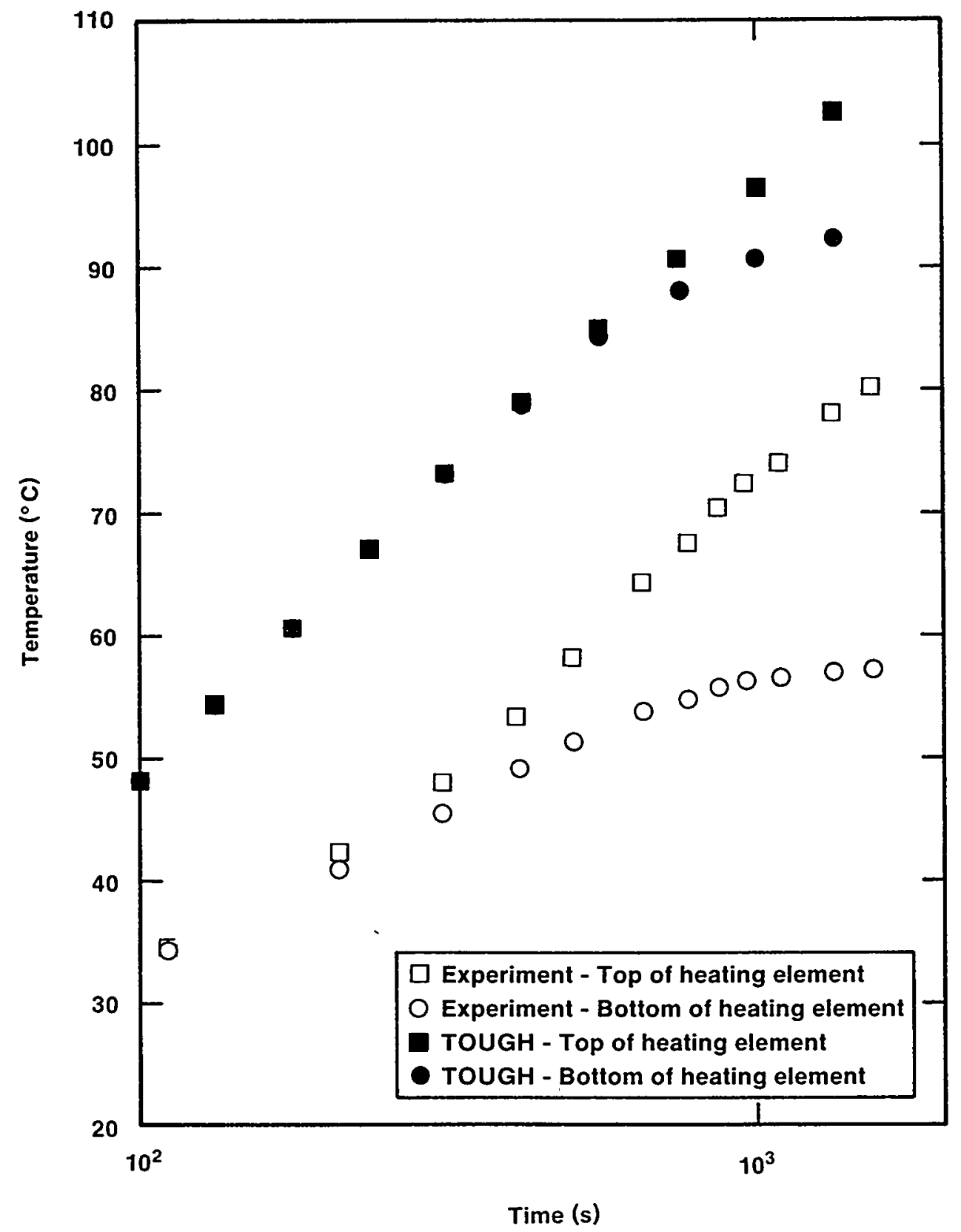

Figure 4.32: Comparison of TOUGH Results with Experimental Results for the Convection Cell Problem 


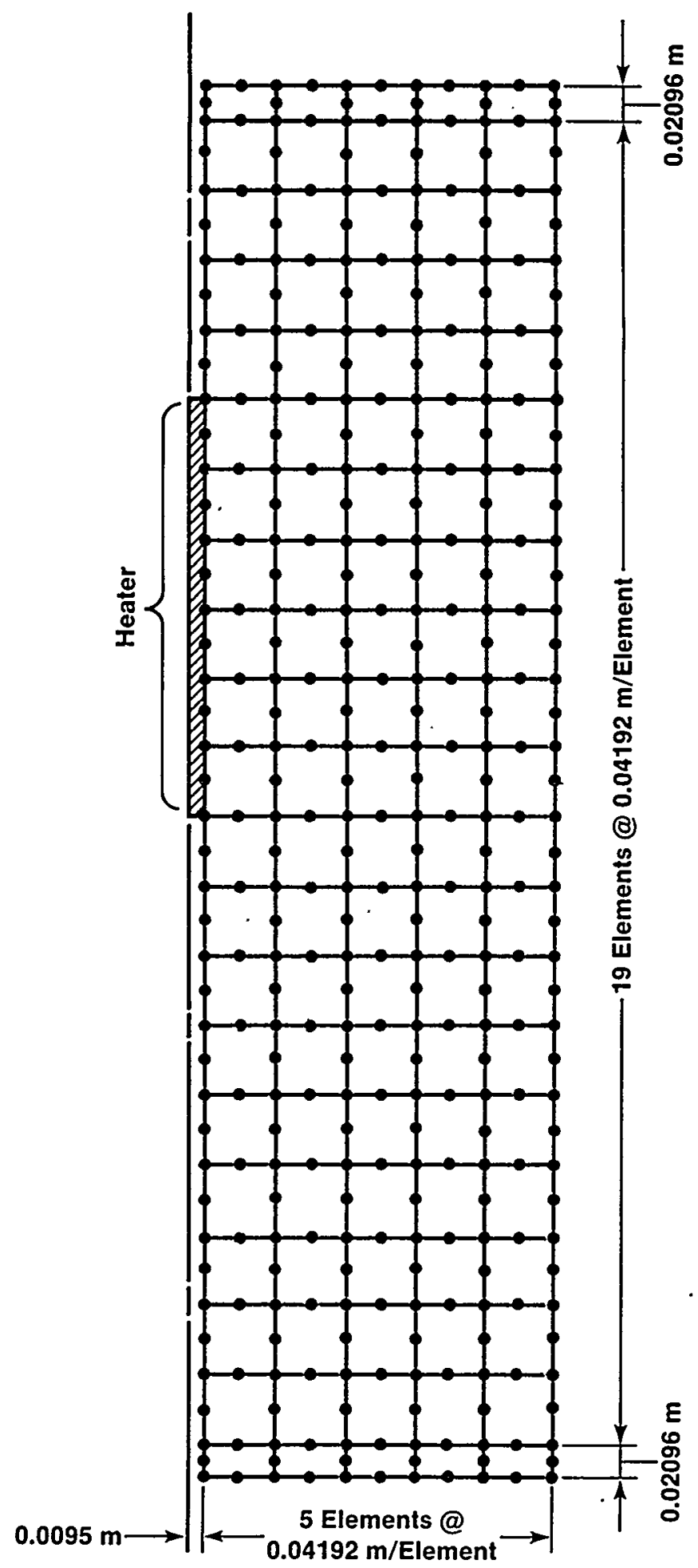

Figure 4.33: NORIA Grid for the Convection Cell Problem 


\subsubsection{Two-Phase Flow}

\subsubsection{TOUGH}

TOUGH could not simulate satisfactorily the two-phase flow problem. Probably the main reason for not obtaining a good solution was the difficulty in handling the boundary condition on the right side and the boundary condition due to placing the core in the oven. TOUGH required 70 time steps and $25 \mathrm{~s}$ of CPU time to generate its solution.

The integrated finite-difference grid used to simulate the problem is presented in Figure 4.34. It consisted of 100 grid blocks labelled AO through A99, each $0.005969 \mathrm{~m}$. wide, and a boundary grid block, labelled YY1, used to control the right side boundary conditions.

On the left side of the grid, zero flux conditions for liquid, gas, and heat were assigned. Because of these conditions, no boundary elements were added at the left side of the grid. On the right side, a large volume element was assigned. This forced a constant pressure, temperature, and gas saturation at that boundary. The pressure, temperature, and gas saturation assigned to this block were $101330 \mathrm{~Pa}, 20^{\circ} \mathrm{C}$, and 1.0 , respectively. The initial conditions were set at a pressure of $1840965 \mathrm{~Pa}$, a liquid saturation of 1.0 , and a linear temperature distribution between $192^{\circ} \mathrm{C}$ at the left edge of the core and $184^{\circ} \mathrm{C}$ at the right edge.

The comparison of the TOUGH results with the experimental results are presented in Figure 4.35. Although TOUGH showed a similar temperature trend as the experiment, TOUGH consistently predicted lower temperatures than those measured in the experiment. The cause of this was threefold and all were related to the boundary conditions.

First, the simulation did not take into account a constant temperature along the side of the core due to placing the core in the oven. This effect would have increased the temperature in the core because the oven temperature was higher than the initial core temperature. Attempts to include a constant temperature boundary condition at the outside edge of the core failed because extremely small time steps were required and, consequently, the solution progressed very slowly making the computer cost prohibitive.

Second, the large volume of the right boundary grid block was inconsistent with the zero temperature gradient boundary condition. As a result, the temperature at the right boundary grid block was kept fixed at $20^{\circ} \mathrm{C}$. This lowered the temperature all through the core which is particularly noticeable at the right side of the plot in Figure 4.35 , where the temperature gradient is very steep. Attempts to force a zero heat flux condition by assigning zero thermal conductivities to the right grid block failed because extremely small time steps resulted.

Third, TOUGH cannot directly handle a transient pressure boundary condition. It must do so with its restart feature. Because the pressure drops very rapidly at the right side, the code would have required a restart at almost every time step. Setting up the input data to repeatedly use the restart feature would have been time consuming and running the code would have been 


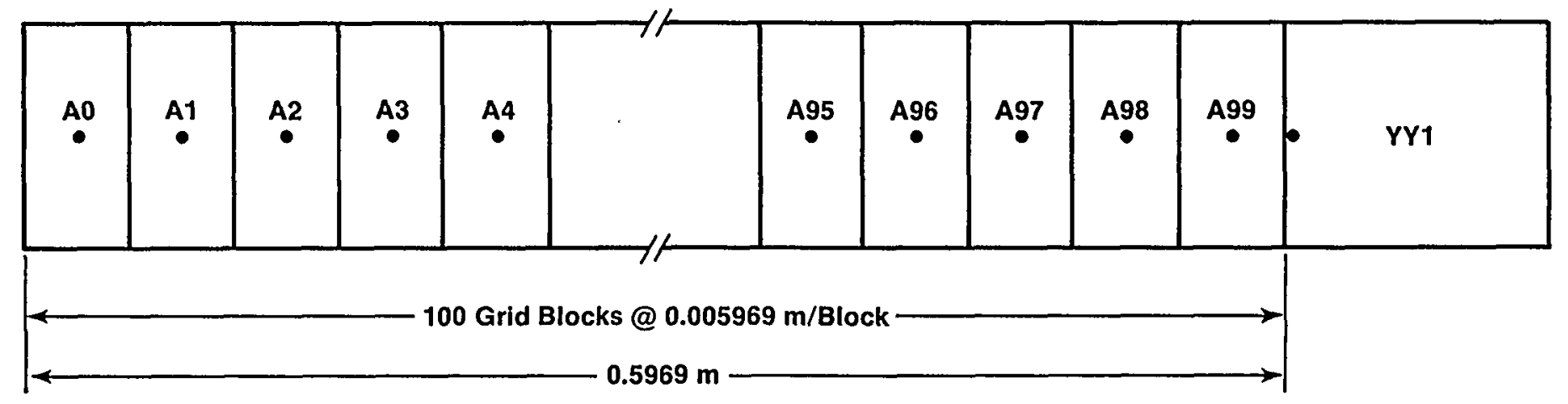

Figure 4.34: TOUGH Grid for the Two-Phase Flow Problem 


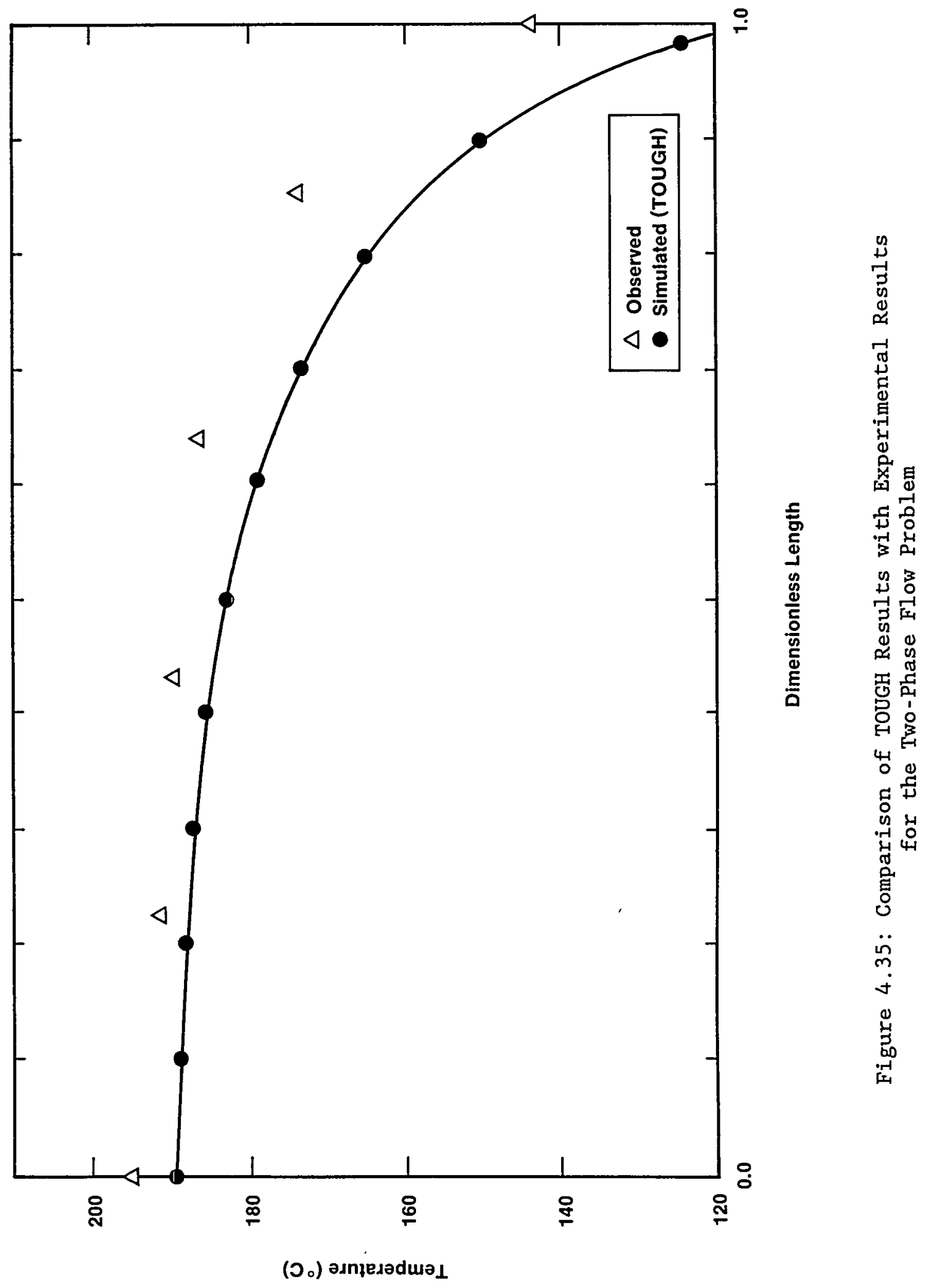


too expensive. However, if the restart feature had been used, heat would not have left the system so quickly and the temperatures would have been higher.

\section{2 .3 .2 NORIA}

The finite-element grid used to simulate the two-phase flow problem with NORIA is presented in Figure 4.36. The grid consisted of 100 elements, each $0.005969 \mathrm{~m}$ wide and $0.0508 \mathrm{~m}$ high. The height of the grid blocks was based on the diameter of the core.

In contrast to the other codes, NORIA can account for the temperature effects of placing the core in the oven by specifying boundary conditions along the top and bottom of rows of nodes on the grid. These nodes were assigned temperatures of $198.9^{\circ} \mathrm{C}$, the oven temperature. In addition, these nodes were also assigned zero values for liquid, vapor, and air mass fluxes. Along the left edge of the grid, zero values were assigned for liquid, vapor, and air mass fluxes, and the conductive heat flux. 'Along the right edge, several types of boundary conditions were applied. First, it was assumed that the liquid flux was zero in order to represent a seepage face. The vapor partial pressure was allowed to vary with time according to the relationship given in Section 3.2.3. The air partial pressure was held fixed at an arbitrarily chosen value of $1000 \mathrm{~Pa}$. Finally, a zero conductive heat flux was assigned along the right side boundary. These conditions implied that heat could escape from the core only by convection of water vapor out the right side boundary. Initial conditions for effective liquid pressure, vapor partial pressure, and air partial pressure were held constant along the length of the core at $2170000 \mathrm{~Pa}, 2170000 \mathrm{~Pa}$, and $1000 \mathrm{~Pa}$, respectively. The initial air temperature varied linearly from $192^{\circ} \mathrm{C}$ at the right end of the core to $184^{\circ} \mathrm{C}$ at the left end.

NORIA could not simulate the two-phase flow problem. After several time steps, NORIA started reducing the time-step size until it became smaller than the initial time-step size. When this situation occurred, execution stopped. The cause for the time step size reduction might be related to the difficulty NORIA has in simulating problems that have no capillary pressure.

Because NORIA cannot directly handle the saturation-capillary pressure curve when there is no capillary pressure, a curve must be forced in the code. In this problem it was done by allowing large saturation changes for extremely small capillary pressure changes by using a linear model of the form:

$$
S=\left(I-P_{c} / a\right)
$$

where $S$ is saturation, $P_{c}$ is capillary pressure, and $a$ is a small number representing the change in capillary pressure between $S=0$ and $S=1$. It can be seen that if $P_{c}$ becomes large due to a calculation that has not converged, say on the first iteration, then $S$ becomes negative and the calculations produce poor results. 


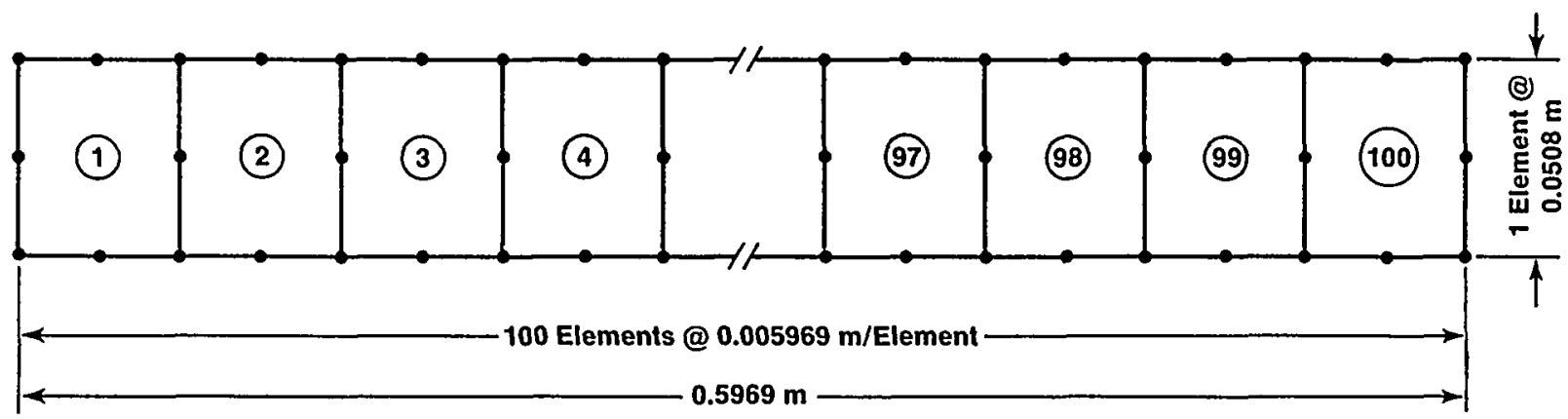

Figure 4.36: NORIA Grid for the Two-Phase Flow Problem 


\subsubsection{PETROS}

The finite-difference grid used to simulate the two-phase flow problem with PETROS is presented in Figure 4.37. It consisted of 101 nodes separated by a distance of $0.005969 \mathrm{~m}$. The left node, node 1, was set so that there was no flux of mass and heat out the system. The right node, node 101, was used to specify several types of boundary conditions. First, a zero flux boundary with respect to both water and heat was used. The zero flux condition approximated a seepage-face boundary condition as the water vaporized very quickly near the right boundary. The zero heat flux condition allowed heat to escape only by convection. In addition, at the right boundary the vapor partial pressure was set to $101330 \mathrm{~Pa}$ to approximate atmospheric conditions. Actually, the pressure at the right boundary was time dependent and approached atmospheric pressure. However, PETROS cannot handle a time dependent boundary pressure condition so the atmospheric pressure was used as a boundary condition. Initial conditions assigned were a saturation of 0.9999 and a temperature distribution of:

$$
T(x)=192.0-13.40258 x
$$

where $\mathrm{x}$ is the distance measured from the left boundary. As in other problems simulated with PETROS, the initial vapor pressure was calculated internally. In this problem, PETROS set the vapor pressure to the saturation pressure. In addition, because PETROS is a one-dimensional code, the impact of placing the core in the oven on temperature could not be simulated. Air was not included in the solution of this problem.

The results from PETROS, presented in Figure 4.38, did not compare well with the experimental results. The temperature at the right side actually increased approximately $1.5^{\circ} \mathrm{C}$ during the simulation when it should have decreased significantly. The temperature at the left side decreased approximately $1.9^{\circ} \mathrm{C}$. Apparently, heat was transferred from the left side of the core to the right side with very little actually leaving the system.

The saturation curve exhibited some odd behavior. The saturation curve produced oscillations, similar to that exhibited by PETROS in the radial boiling front problem (Section 4.1.4.3), throughout the modeled region. The cause for this behavior is not known but may be due to the specification in the problem of no capillary pressure. This specification caused zero divide errors when implemented in PETROS. To remedy this, a linear capillary pressure curve, similar to the one used by NORIA in the preceding section, was specified. This might be producing large capillary pressures causing an ill-conditioning of the finite-difference equation which results in oscillations. 


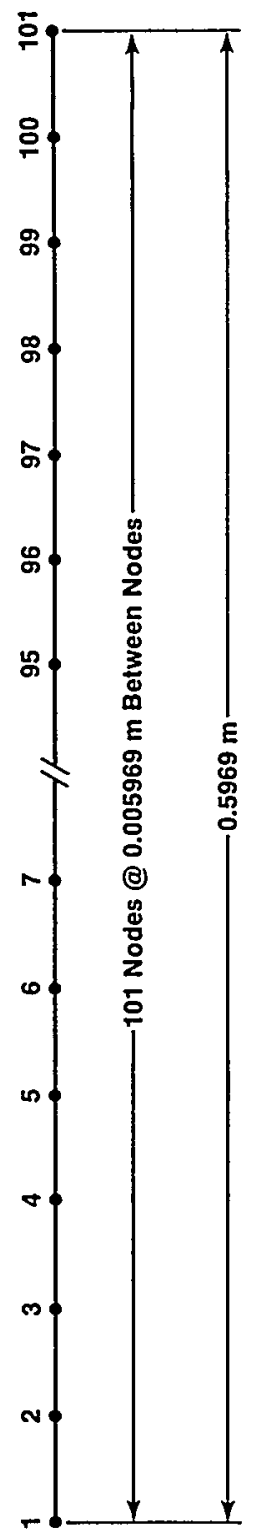

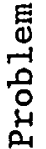

$\stackrel{3}{0}$

פ

竞

总

ఖ

莒

年

용

鼠

A.

$\ddot{n}$

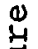

岁

䍃 


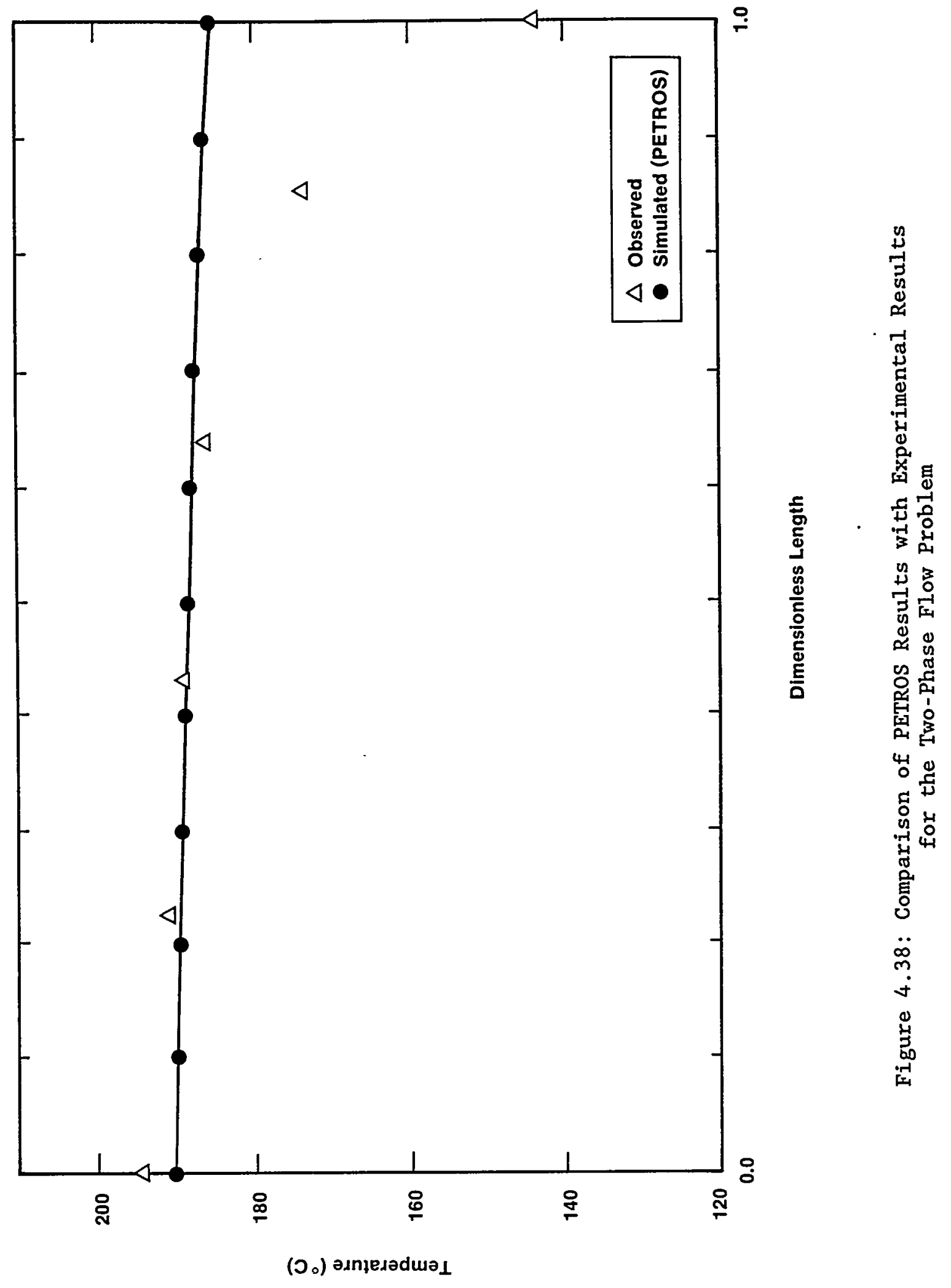


.... 
CHAPTER 5

CONCLUSIONS AND RECOMMENDATIONS

\subsection{Conclusions}

Several conclusions can be reached from this study. In general, the codes reviewed, TOUGH, NORIA, and PETROS, are extremely difficult to use. First, the codes have their own built-in time-step-size criteria which on more than one occasion prevented the codes from running to completion even for very simple one-dimensional problems. Second, each code seems to have some peculiarity in handling boundary conditions, the result of the numerical algorithm employed in the code or poor documentation. This second item is presented below in slightly more detail. Third, all the codes seemed to have some difficulty with evaporation/condensation problems. Fourth, there does not seem to be a wealth of problems available for adequately testing these codes for conditions that may occur near an HLW repository. The result of each simulation is summarized in Table 5.1 .

\subsubsection{TOUGH}

TOUGH, in general, was capable of solving most of the problems, and produced some type of solution for all the problems. Overall, with respect to solving problems, it performed the best of the three codes tested. The problems it had difficulty solving were caused by the convergence problems on the dependent variables, which resulted in small time-step sizes, and boundary conditions. It also had the most difficulty with numerical dispersion when the heat transport problems were tested. Numerical dispersion could have been avoided if a finer mesh had been used when running this problem. Typically, core storage requirements were very large, even for small problems. Time requirements for solving problems covered a large range. Smal1, well-behaved problems, those that used few grid blocks or did not include vaporization, ran fairly quickly, even rivalling PETROS' times on some problems. On other problems, the large ones or ones that involve evaporation, long running times were necessary.

TOUGH's greatest weakness is probably the way it handles boundary conditions. The method employed in TOUGH to handle boundary conditions (large volumes elements or the lack of adjoining elements) limits its use when boundary conditions are mixed in the form of prescribed mass fluxes and constant temperatures or something similar. These boundary conditions are inconsistent with each other when being implemented with the TOUGH method.

Another weakness in TOUGH is the method employed to enter the grid information. The specifying of grid volumes and interfacial areas between grid blocks is cumbersome when specifying a problem. of course, this helps with making TOUGH a one-, two-, or three-dimensional code. The weakness can be overcome with rectangular type grids and/or mesh generators.

The governing equations in TOUGH are fairly general. The code solves only three equations, gas pressure, mass fraction, and temperature, while PETROS 
Table 5.1: Summary of Code Comparisons

ANALYTICAL/NUMERICAL SOLUTIONS

Code

$\begin{array}{clll}\text { Problem } & \text { TOUGH } & \text { NORIA } & \text { PETROS } \\ 1 & \text { solved } & \text { solved } & \text { solved } \\ 2 & \begin{array}{l}\text { solved with } \\ \text { some numerical } \\ \text { dispersion }\end{array} & \text { solved } & \text { solved } \\ 3 & \begin{array}{l}\text { solved for } \\ 1.5 \times 10^{6} \mathrm{~s}\end{array} & \begin{array}{l}\text { solved for } \\ 4.6 \times 10^{6} \mathrm{~s}\end{array} & \text { solved } \\ 4 & \text { solved } & \begin{array}{l}\text { small time-step } \\ \text { sizes }\end{array} & \begin{array}{l}\text { poor solution; } \\ \text { oscillating } \\ \text { saturation }\end{array} \\ 5 & \text { solved } & \begin{array}{l}\text { small time-step } \\ \text { sizes }\end{array} & \text { poor convergence }\end{array}$

EXPERIMENTAL RESULTS

Code

$\begin{array}{llll}\text { Problem } & \text { TOUGH } & \text { NORIA } & \text { PETROS } \\ 1 & \text { solved for } 6300 \mathrm{~s} & \begin{array}{l}\text { small time-step } \\ \text { sizes }\end{array} & 1-\mathrm{d} \text { code } \\ 2 & \text { incorrect.solution } & \begin{array}{l}\text { small time-step } \\ \text { sizes }\end{array} & 1-\mathrm{d} \text { code } \\ 3 & \begin{array}{l}\text { incorrect solution } \\ \text { due to poor } \\ \text { boundary condition } \\ \text { handling }\end{array} & \begin{array}{l}\text { small time-step } \\ \text { sizes }\end{array} & \begin{array}{l}\text { incorrect solution; } \\ \text { oscillating } \\ \text { saturations }\end{array}\end{array}$


and NORIA solve four. This may result in its overall ability to solve the comparison problems; it has fewer equations on which to fail. The governing equations, however, do not consider Knudsen diffusion, which may be important in rock with small pores such as tuff, or thermodiffusion (the transport of one gas through the other due to temperature gradients).

\section{1 .2 NORIA}

NORIA had difficulty solving over half the problems tested. Its difficulty came mainly from its built-in time-step-size criteria. In the cases where NORIA could not solve the problem, its time-step size would increase for several time steps and then decrease until it became smaller than the first time step. This would cause NORIA to stop running. However, when NORIA produced results, they were extremely accurate, the result of the use of quadratic basis functions and small time steps. However, the cost of this accuracy was high. The NORIA simulations typically took the longest of the three codes tested. Even simple small problems, such as the horizontal infiltration problem, required more than one-half hour of computer time to complete.

When specifying input data, NORIA was fairly easy to use. Data are just listed in order, with blanks if default values are used, and NORIA processes the rest. There was some difficulty with the use of mass flux boundary conditions. The documentation indicated that fluxes be specified as velocity but did not specify if they were Darcy or pore velocities. The two-dimensional horizontal infiltration problem, which could have been used to test for the proper velocity, could not be successfully run.

The governing physics in NORIA are based on a liquid pressure, a vapor partial pressure, an air partial pressure, and a heat equation. The properties of water, air, and vapor can be specified by the user as either constants or variables through subprograms. However, the density of water is always a constant. The compressibility and thermal expansivity of the soll/rock can be handled with a user supplied subroutine. In addition, the Boussinesq approximation is used to handle density-driven flow caused by heat.

\subsubsection{PETROS}

PETROS was capable of correctly simulating less than half the problems tested and produced poor solutions for several others. The remaining problems could not be simulated because of time-step problems and the method used to handle vapor and air boundary conditions. In the four cases that PETROS correctly simulated, the solution was very accurate. It produced the smallest running times of the three codes tested, probably the result of it being a one-dimensional code.

When PETROS had a time-step-size problem, it was because it produced small time steps. It did not have the problem that NORIA had where time-step size would oscillate and then become very small. 
The method used in PETROS to handle vapor and air boundaries was difficult to implement. First, the air and vapor boundary conditions depended on each other and a $2 \times 2$ matrix was set up to handle this dependence. However, the documentation was not clear on what went into the matrix when flux conditions were implemented. This may have been the reason why the radial boiling front and two-phase flow problems could not be simulated.

PETROS solves similar equations as NORIA, but uses a different formulation for them. Thermodiffusion is neglected and many of the water, air, and vapor properties are treated as constants.

\subsection{Recommendations}

From this study it can be seen that each code exhibits one weakness or another, whether it be boundary conditions (TOUGH,PETROS), core storage (TOUGH), or computation time (NORIA). In addition, none of the codes can directly handle fractures, a feature of welded tuff formations. Also, it is not known how long the effects of heat generated from an HLW repository will affect the flow field around the repository.

It is recommended that an investigation be made into the exact effects heat may have on the flow field around an HLW repository. This investigation may consist of either a literature search, a modeling study, or a field study.

It is also recommended that, if the results of the investigation proposed above indicate a significant impact on the flow field caused by heat generation, a new strongly heat-driven flow code be developed. This new code would implement the best features of the codes tested in this study and eliminate the worst. In addition, it would also include the capability to directly handle fractures in the unsaturated zone. TOUGH and NORIA can model fractures, but this is done explicitly by giving the fractures their own grid blocks or finite elements. TOUGH can probably also model fractures with the GMINC method (Pruess and Narasimhan, 1985).

The code might be based on the physics presented in TOUGH, but include Knudsen diffusion and thermodiffusion, because TOUGH uses fewer equations and seemed to be able to solve most of the problems. The method of handling boundary conditions should be based on the methods presented in NORIA. These boundary conditions were quite easy to implement. Finally, the speed of PETROS should be implemented if possible. This may be difficult since PETROS is a one-dimensional code which uses a tridiagonal algorithm to solve its equations. The tridiagonal algorithm approach may be implemented in the new code by some type of iterative directional solution technique which can take advantage of the fast convergence of Newton-Raphson iterations. Conjugate gradient techniques should also be considered as a possible method for solving the equations. Conjugate gradient methods require smaller amounts of memory and seem to be faster than other methods (Bear and Verruijt, 1987)

In Section 5.1 it was mentioned that there is a lack of test problems with experimental results. Therefore, it is also recommended that some experiments be designed and performed to simulate conditions that would occur around an HLW repository. The results of these experiments should be used to test strongly heat-driven flow codes. 
CHAPTER 6

REFERENCES

Avdonin, N. A., "Some Formulas for Calculating the Temperature Field of a Stratum Subject to Thermal Injection, "Neft'i Gaz 3, 37-41 (1964).

Bear, J., and A. Verruijt, Modeling Groundwater Flow and Pollution, 414 pp., D. Reidel Publishing Co., Boston MA, 1987.

Bixler, N. E., "NORIA - A Finite Element Computer Program for Analyzing Water, Vapor, and Energy Transport in Porous Media," SAND84-2057, Sandia National Laboratories, Albuquerque NM, August 1985.

Duff, I. S., "MA28- A Set of FORTRAN Subroutines for Sparse Unsymmetric Linear Equations," Report AERE R 8730, U. K. Atomic Energy Authority, Harwe11 Laboratories, United Kingdom, 1977.

Faust, C. R., and J. W. Mercer, "Geothermal Reservoir Simulation 2. Numerical Solution Techniques for Liquid- and Vapor-Dominated Hydrothermal Systems," Water Resources Research 15, 31-46 (1979).

Garg, S. K., "Pressure Transient Analysis for Two-Phase (Water/Steam) Geothermal Reservoirs," Society of Petroleum Engineers Journal, 206-214, June 1980.

Hadley, G. R., "PETROS - A Program for Calculating Transport of Heat, Water, Water Vapor, and Air Through a Porous Material," SAND84-0878, Sandia National Laboratories, Albuquerque NM, May 1985.

International Formulation Committee, "A Formulation of the Thermodynamic Properties of Ordinary Water Substance," IFC Secretariat, Dusseldorf Germany, 1967.

Kruger, P., and H.V J. Ramey, "Stimulation and Reservoir Engineering of Geothermal Resources," Stanford Geothermal Program Report SGR-TR-1, Stanford University, Stanford CA, June 1974.

Meyer, C. A., R. B. McClintock, G. J. Silvester, and R. C. Spencer, "1967 ASME Steam Tables," $2^{\text {nd }}$ edition, American Society of Mechanical Engineers, NY, 1968. 
Oster, C. A., "Review of Ground-Water Flow and Transport Models in the Unsaturated Zone," NUREG/CR-2917, PNL-4427, Pacific Northwest Laboratory, Richland, WA, November 1982.

Phillip, J. R., "Numerical Solution of Equations of the Diffusive Type with Diffusivity Concentration Dependent," Transactions, Faraday Society 51 , 885-892, (1955).

Pruess, K., "TOUGH User's Guide," NUREG/CR-4645, SAND86-7104, LBL-20700, Sandia National Laboratories, Albuquerque, NM, August 1987.

Pruess, K., and T. N. Narasimhan, "A Practical Method for Modeling Fluid and Heat Flow in Fractured Porous Media," Society of Petroleum Engineers Journal 25(1), 14-26, (1985).

Reda, D. C., "Natural Convection Experiments about a Finite-Length Cylindrical Heat Source in a Liquid-Saturated Porous Medium," SAND83-2209C, Sandia National Laboratories, Albuquerque NM, November 1984.

Reeves, M., D. S. Ward, N. D. Johns, and R. M. Cranwell, "Theory and Implementation for SWIFT II, The Sandia Waste-Isolation Flow and Transport Model for Fractured Media, Release 4.84," NUREG/CR-3328, SAND83-1159, Sandia National Laboratories, Albuquerque NM, August 1986.

Ross, B., J. W. Mercer, S. D. Thomas, and B. H. Lester, "Benchmark Problems for Repository Siting Models," NUREG/CR-3097, Geotrans, Inc., Reston, VA, December 1982 .

Ude1l, K. S., and J. S. Fitch, "Heat and Mass Transfer in Capillary Porous Media Considering Evaporation, Condensation and Non-Condensible Gas Effects," paper presented at the $23^{\text {rd }}$ ASME/AIChE National Heat Transfer Conference, Denver CO, 103-110, American Society of Mechanical Engineers, New York, August 1985.

Vauclin, M., D. Khanji, and G. Vachaud, "Experimental and Numerical Study of a Transient, Two-Dimensional Unsaturated-Saturated Water Table Recharge Problem, " Water Resources Research 15, 1089-1101, (1979).

Ward, D. S., M. Reeves, and L. E. Duda, "Verification and Field Comparison of the Sandia Waste-Isolation Flow and Transport Model (SWIFT)," NUREG/CR-3316, SAND83-1154, Sandia National Laboratories, Albuquerque, NM, April 1984. 
DISTRIBUTION

(RESEARCH)

G. F. Birchard

MS NL/S-260

U. S. Nuclear Regulatory Commission

Washington, DC 20555

Emmy Booy

MS NL/S-260

U. S. Nuclear Regulatory Commission

Washington, DC 20555

Richard Gri11

MS NL/S-260

U. S. Nuclear Regulatory Commission Washington, DC 20555

Mark Haisfield

MS NL/S-260

U. S. Nuclear Regulatory Commission Washington, DC 20555

R. Kornasiewicz

MS NL/S-260

U. S. Nuclear Regulatory Commission Washington, DC 20555

Linda Kovach

MS NL/S-260

U. S. Nuclear Regulatory Commission Washington, DC 20555

Janet Lambert

MS NL/S-260

U. S. Nuclear Regulatory Commission Washington, DC 20555

T. J. McCartin

MS NL/S-260

U. S. Nuclear Regulatory Commission Washington, DC 20555

T. J. Nicholson,

MS NL/S-260

U. S. Nuclear Regulatory Commission Washington, DC 20555

E. O'Donne11

MS NL/S-260

U. S. Nuclear Regulatory Commission Washington, DC 20555 
W. Ott

MS NL/S -260

U. S. Nuclear Regulatory Commission Washington, DC 20555

Jacob Philip

MS NL/S-260

U. S. Nuclear Regulatory Commission Washington, DC 20555

Clark Prichard

MS NL/S-260

U. S. Nuclear Regulatory Commission Washington, DC 20555

J. D. Randall

MS NL/S-260

U. S. Nuclear Regulatory Commission Washington, DC 20555

P. Reed

MS NL/S-260

U. S. Nuclear Regulatory Commission Washington, DC 20555

Mel Silberberg

MS NL/S-260

U. S. Nuclear Regulatory Commission Washington, DC 20555

Martin Weinstein

MS NL/S - 260

U. S. Nuclear Regulatory Commission Washington, DC 20555

(NMSS HLW)

Charlotte Abrams

MS 4-H-3

U. S. Nuclear Regulatory Commission Washington, DC 20555

P. Altomare

MS 4- $\mathrm{H}-3$

U. S. Nuclear Regulatory Commission Washington, DC 20555

Ronald Ballard

MS 4-H-3

U. S. Nuclear Regulatory Commission Washington, DC 20555 
William Belke

MS 4-H-3

U. S. Nuclear Regulatory Commission

Washington, DC 20555

Michael Blackford

MS 4-H-3

U. S. Nuclear Regulatory Commission Washington, DC 20555

John Bradbury

MS 4-H-3

U. S. Nuclear Regulatory Commission Washington, DC 20555

David Brooks

MS 4- $\mathrm{H}-3$

U. S. Nuclear Regulatory Commission Washington, DC 20555

Pauline Brooks

MS 4-H-3

U. S. Nuclear Regulatory Commission Washington, DC 20555

R. E. Browning

MS 4-H-3

U. S. Nuclear Regulatory Commission Washington, DC 20555

John T. Buckley

MS 4-H-3

U. S. Nuclear Regulatory Commission Washington, DC 20555

Joseph Bunting

MS $4-\mathrm{H}-3$

U. S. Nuclear Regulatory Commission Washington, DC 20555

Tom Cardone

MS 4-H-3

U. S. Nuclear Regulatory Commission Washington, DC 20555

Document Control Center, DHLWM

MS 4-H-3

U. S. Nuclear Regulatory Commission Washington, DC 20555

K. C. Chang

MS 4-H-3

U. S. Nuclear Regulatory Commission Washington, DC 20555 
Donald L. Chery, Jr. MS 4-H-3

U. S. Nuclear Regulatory Commission Washington, DC 20555

R. B. Codell

MS $4-\mathrm{H}-3$

U. S. Nuclear Regulatory Commission W- i.ngton, DC 20555

Neil Coleman

MS 4- $\mathrm{H}-3$

U. S. Nuclear Regulatory Commission Washington, DC 20555

Seth Coplan

MS 4-H-3

U. S. Nuclear Regulatory Commission Washington, DC 20555

Julia Corrado

MS 4-H-3

U. S. Nuclear Regulatory Commission Washington, DC 20555

Mark Delligatti

MS 4-H-3

U. S. Nuclear Regulatory Commission Washington, DC 20555

James P. Donnelly

MS 4-H-3

U. S. Nuclear Regulatory Commission Washington, DC 20555

Alan B. Duncan.

MS 4-H-3

U. S. Nuclear Regulatory Commission Washington, DC 20555

D. J. Fehringer

MS 4-H-3

U. S. Nuclear Regulatory Commission Washington, DC 20555

William Ford

MS 4-H-3

U. S. Nuclear Regulatory Commission Washington, DC 20555

Shirley Fortuna

ISS 4-H-3

U. S. Nuclear Regulatory Commission Washington, DC 20555 
Danie1 M. Gillen

MS 4-H-3

U. S. Nuclear Regulatory Commission

Washington, DC 20555

Vanessa Gland

MS 4-H-3

U. S. Nuclear Regulatory Commission

Washington, DC 20555

Chad Glenn

MS 4-H-3

U. S. Nuclear Regulatory Commission

Washington, DC 20555

Paul Goldberg

MS 4-H-3

U. S. Nuclear Regulatory Commission Washington, DC 20555

Dinesh Gupta

MS 4-H-3

U. S. Nuclear Regulatory Commission Washington, DC 20555

A. K. Ibrahim

MS 4-H-3

U. S. Nuclear Regulatory Commission Washington, DC 20555

Robert Johnson

MS 4-H-3

U. S. Nuclear Regulatory Commission Washington, DC 20555

P. S. Justus

MS 4-H-3

U. S. Nuclear Regulatory Commission Washington, DC 20555

Kenneth Kalman

MS 4-H-3

U. S. Nuclear Regulatory Commission Washington, DC 20555

James E. Kennedy

MS 4-H-3

U. S. Nuclear Regulatory Commission Washington, DC 20555

Michael Lee

MS 4-H-3

U. S. Nuclear Regulatory Commission Washington, DC 20555 
Harold Lefevre

MS 4-H-3

U. S. Nuclear Regulatory Commission Washington, DC 20555

\section{Yang $\mathrm{Li}$}

MS 4-H-3

U. S. Nuclear Regulatory Commission Washington, DC 20555

John J . Linehan

MS 4- $\mathrm{H}-3$

U. S. Nuclear Regulatory Commission Washington, DG 20555

Robert MacDougal1

MS 4- $\mathrm{H}-3$

U. S. Nuclear Regulatory Commission Washington, DC 20555

Kimberly Malone

MS 4-H-3

U. S. Nuclear Regulatory Commission Washington, DC 20555

Jernee Martin

MS 4-H-3

U. S. Nuclear Regulatory Commission Washington, DC 20555

Keith McConnell

MS 4-H-3

U. S. Nuclear Regulatory Commission Washington, DC 20555

Tin Mo

MS 4-H-3

U. S. Nuclear Regulatory Commission Washington, DC 20555

M. Nataraja

MS 4-H-3

U. S. Nuclear Regulatory Commission Washington, DC 20555

Robert Neel

MS 4-H-3

U. S. Nuclear Regulatory Commission Washington, DC 20555

Jerome Pearring

MS 4- $\mathrm{H}-3$

U. S. Nuclear Regulatory Commission Washington, DC 20555 
John J. Peshel

MS 4-H-3

U. S. Nuclear Regulatory Commission

Washington, DC 20555

Charles H. Peterson

MS 4-H-3

U. S. Nuclear Regulatory Commission

Washington, DC 20555

J. Pohle

MS 4-H-3

U. S. Nuclear Regulatory Commission

Washington, DC 20555

Linda Riddle

MS 4-H-3

U. S. Nuclear Regulatory Commission

Washington, DC 20555

Frederick W. Ross

MS 4-H-3

U. S. Nuclear Regulatory Commission

Washington, DC 20555

Newton Kingman Stablein

MS 4- $\mathrm{H}-3$

U. S. Nuclear Regulatory Commission

Washington, DC 20555

Barbara Stiltenpole

MS 4-H-3

U. S. Nuclear Regulatory Commission

Washington, DC 20555

Eileen Tana

MS 4-H-3

U. S. Nuclear Regulatory Commission

Washington, DC 20555

Naiem Tanious

MS 4-H-3

U. S. Nuclear Regulatory Commission

Washington, DC 20555

Anthony J. Tesoriero

MS 4-H-3

U. S. Nuclear Regulatory Commission

Washington, DC 20555

Brian Thomas

MS 4-H-3

U. S. Nuclear Regulatory Commission

Washington, DC 20555 
David Tiktinsky

MS 4-H-3

U. S. Nuclear Regulatory Commission Washington, DC 20555

John Trapp

MS 4-H-3

U. S. Nuclear Regulatory Commission Washington, DC 20555

Tilak Verma

MS 4-H-3

U. S. Nuclear Regulatory Commission Washington, DC 20555

Wayne Walker

MS 4-H-3

U. S. Nuclear Regulatory Commission Washington, DC 20555

Jim Warner

MS 4-H-3

U. S. Nuclear Regulatory Commission Washington, DC 20555

Richard Weller

MS 4-H-3

U. S. Nuclear Regulatory Commission Washington, DC 20555

Lisa Williams

MS 4- $\mathrm{H}-3$

U. S. Nuclear Regulatory Commission Washington, DC 20555

B. Joe Youngblood

MS 4-H-3

U. S. Nuclear Regulatory Commission Washington, DC 20555

(NMSS LLW)

Sher Bahadur

MS 5-E-4

U. S. Nuclear Regulatory Commission Washington, DC 20555

M. J. Bell

MS 5-E-4

U. S. Nuclear Regulatory Commission Washington, DC 20555 
Susan Bilhorn

MS 5-E-4

U. S. Nuclear Regulatory Commission

Washington, DC 20555

Regis Boyle

MS 5-E-4

U. S. Nuclear Regulatory Commission

Washington, DC 20555

Louis Bykoski

MS 5-E-4

U. S. Nuclear Regulatory Commission

Washington, DC 20555

Lynn Deering

MS 5-E-4

U. S. Nuclear Regulatory Commission Washington, DC 20555

Kitty S. Dragonette

MS 5-E-4

U. S. Nuclear Regulatory Commission Washington, DC 20555

Myron Fliegle

MS 5-E-4

U. S. Nuclear Regulatory Commission

Washington, DC 20555

Daniel Gillen

MS 5-E-4

U. S. Nuclear Regulatory Commission Washington, DC 20555

Chad Glenn

MS 5-E-4

U. S. Nuclear Regulatory Commission Washington, DC 20555

G. N. Gnugnoli

MS 5-E-4

U. S. Nuclear Regulatory Commission Washington, DC 20555

J. Greeves

MS 5-E-4

U. S. Nuclear Regulatory Commission Washington, DC 20555

Joel Grimm

MS 5-E-4

U. S. Nuclear Regulatory Commission Washington, DC 20555 
Anthony Huffert

MS 5-E-4

U. S. Nuclear Regulatory Commission

Washington, DC 20555

Banad Jagannath

MS 5-E-4

U. S. Nuclear Regulatory Commission

Washington, DC 20555

Ted L. Johnson

MS 5-E-4

U. S. Nuclear Regulatory Commission Washington, DC 20555

Tim Johnson

MS 5-E-4

U. S. Nuclear Regulatory Commission Washington, DC 20555

Timothy C. Johnson

MS 5-E-4

U. S. Nuclear Regulatory Commission Washington, DC 20555

Samuel Jones

MS 5-E-4

U. S. Nuclear Regulatory Commission Washington, DC 20555

Joseph Kane

MS 5-E-4

U. S. Nuclear Regulatory Commission Washington, DC 20555

M. R. Knapp

MS 5-E-4

U. S. Nuclear Regulatory Commission Washington, DC 20555

Janet Lepre

MS $5-E-4$

U. S. Nuclear Regulatory Commission Washington, DC 20555

Paul Lohaus

MS 5-E-4

U. S. Nuclear Regulatory Commission Washington, DC 20555

D. Martin

MS 5-E-4

U. S. Nuclear Regulatory Commission Washington, DC 20555 
Keith McDaniel

MS 5-E-4

U. S. Nuclear Regulatory Commission

Washington, DC 20555

George Pangburn

MS 5-E-4

U. S. Nuclear Regulatory Commission

Washington, DC 20555

LeRoy S. Person

MS 5-E-4

U. S. Nuclear Regulatory Commission

Washington, DC 20555

C. L. Pittiglio

MS 5-E-4

U. S. Nuclear Regulatory Commission

Washington, DC 20555

Gary W. Roles

MS $5-\mathrm{E}-4$

U. S. Nuclear Regulatory Commission

Washington, DC 20555

Mary Jo Seeman

MS 5-E-4

U. S. Nuclear Regulatory Commission

Washington, DC 20555

James A. Shaffner

MS 5-E-4

U. S. Nuclear Regulatory Commission

Washington, DC 20555

Dennis Sollenberger

MS 5-E-4

U. S. Nuclear Regulatory Commission

Washington, DC 20555

R. J. Starmer

MS 5-E-4

U. S. Nuclear Regulatory Commission

Washington, DC 20555

John J, Surmeier

MS 5-E-4

U. S. Nuclear Regulatory Commission

Washington, DC 20555

James D. Thomas

MS 5-E-4

U. S. Nuclear Regulatory Commission

Washington, DC 20555 
Michael Tokar

MS 5-E-4

U. S. Nuclear Regulatory Commission

Washington, DC 20555

Sandra Wastler

MS 5-E-4

U. S. Nuclear Regulatory Commission

Washington, DC 20555

M. F. Weber

MS 5-E-4

U. S. Nuclear Regulatory Commission

Washington, DC 20555

Kristin Westbrook

MS 5-E-4

U. S. Nuclear Regulatory Commission

Washington, DC 20555

E. A. Wick

MS 5-E-4

U. S. Nuclear Regulatory Commission

Washington, DC 20555

D. A. Widmayer

MS 5-E-4

U. S. Nuclear Regulatory Commission

Washington, DC 20555

Yvonne Young

MS 5-E-4

U. S. Nuclear Regulatory Commission

Washington, DC 20555

(CONTRACTORS AND CONSULTANTS)

J. J. K. Daemen

Department Mining and Geological Engineering

University of Arizona

Tucson, AZ 85721

Frank Dodge

Center for Nuclear Waste Regulatory Analyses

Southwest Research Institute

6220 Culebra Rd.

P.O. Drawer 28510

San Antonio, TX 78284

D. D. Evans

Department of Hydrology and Water Resources

University of Arizona

Tucson, AZ 85721 
Christopher Frietas

Center for Nuclear Waste Regulatory Analyses

Southwest Research Institute

6220 Culebra Rd.

P.O. Drawer 28510

San Antonio, TX 78284

Professor Lynn W. Gelhar

MIT

Department of Civil Engineering

Room 48-329

Cambridge, MA 02139

John Gibbons

Remote Sensing Systems

4300 San Mateo Blvd., NE

Albuquerque, NM 87110

Michael T. Goodrich

GRAM, Inc.

1709 Moon St., NE

Albuquerque, NM 87112

Francis A. Kulacki

Office of the Dean

College of Engineering

Colorado State University

Fort Collins, CO 80523

Paul A. Mattingly

Science Applications International Corp.

2109 Air Park Rd., SE

Albuquerque, NM 87106

Dennis McLaughlin

MIT

Department of Civil Engineering

Room 48-329

Cambridge, MA 02139

Prasad K. Nair

Center for Nuclear Waste Regulatory Analyses

Southwest Research Institute

6220 Culebra Rd.

P.O. Drawer 28510

San Antonio, TX 78284

Shlomo P. Neuman

Department of Hydrology and Water Resources

University of Arizona

Tucson, Arizona 85721 
Gretchen C. Newman

GRAM, Inc.

1709 Moon St., NE

Albuquerque, NM 87112

Alva M. Parsons

GRAM, Inc.

1709 Moon St., NE

Albuquerque, NM 87112

Wesley C. Patrick

Center for Nuclear Waste Regulatory Analyses

Southwest Research Institute

6220 Culebra Rd.

P.O. Drawer 28510

San Antonio, TX 78284

Mel G. Piepho

Technadyne Engineering Consultants, Inc.

P.O. Box 13928

Albuquerque, NM 87192

John Russe11

Center for Nuclear Waste Regulatory Analyses

Southwest Research Institute

6220 Culebra Rd.

P.O. Drawer 28510

San Antonio, TX 78284

Kenneth E. Torrance

Sibley School of Mechanical and Aerospace Engineering Upson and Grumman Halls

Cornell University

Ithaca, NY 14850

C. David Updegraff

GRAM, Inc.

1709 Moon St., NE

Albuquerque, NM 87112

Krishan $\mathrm{K}$. Wahi

GRAM, Inc.

1709 Moon St., NE

Albuquerque, NM

SANDIA NATIONAL LABORATORIES

1511 R. Eaton

3141 S. A. Landenberger (5)

3151 W. I. Klein

6312 M. E. Fewel1

6400 D. J. McCloskey

6410 D. A. Dalgren

6416 E. J. Bonano 
6416 M. S. Y. Chu

6416 P. A. Davis

6416 D. P. Gallegos(32)

6416 C. P. Harlan

6416 M. W. Kozak

6416 N. E. O'lague

6416 L. L. P. Price

8524 J. A. Wackerly 


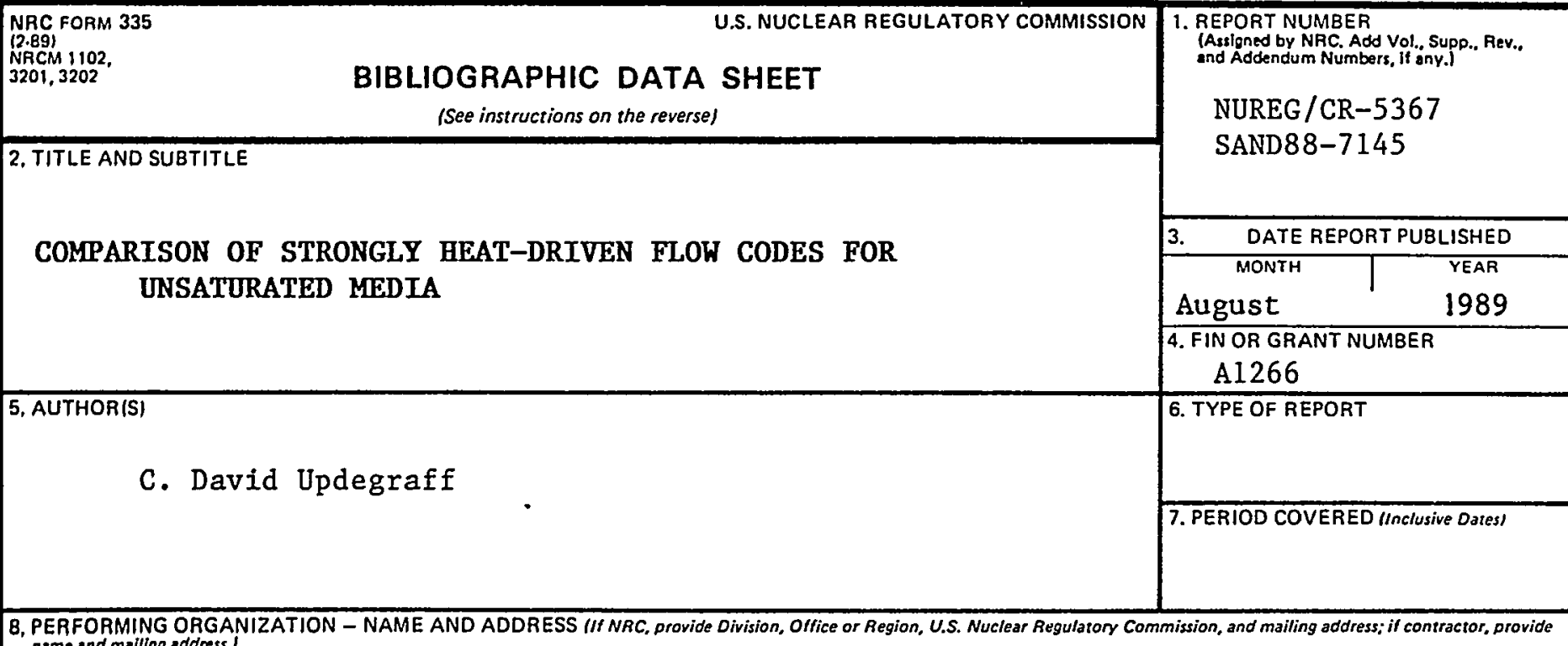
namo and mailing address.)

GRAM, Inc.

1709 Moon St. NE

Albuquerque, NM 87112
Under Contract to:

Sandia National Laboratories

Albuquerque, NM 87185

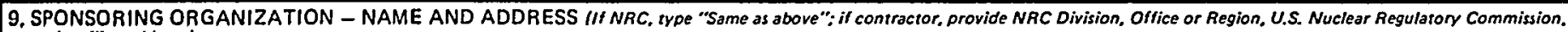
and malling address.)

Division of Engineering

Office of Nuclear Regulatory Research

U.S. Nuclear Regulatory Commission

Washington, DC 20555

10. SUPPLEMENTARY NOTES

11, ABSTRACT (200 words or less)

Under the sponsorship of the U.S. Nuclear Regulatory Commission, Sandia National Laboratories (SNL) is developing a performance assessment methodology for the analysis of long-term disposal of high-level radioactive waste (HLW) in unsaturated welded tuff. As part of this effort, SNL evaluated existing strongly heat-driven flow computer codes for simulating ground-water flow in unsaturated media. The three codes tested, NORIA, PETROS, and TOUGH, were compared against a suite of problems for which analytical and numerical solutions or experimental results exist. The problems were selected to test the abilities of the codes to simulate situations ranging from simple, uncoupled processes, such as two-phase flow or heat transfer, to fully coupled processes, such as vaporization caused by high temperatures. In general, all three codes were found to be difficult to use because of (1) built-in time stepping criteria, (2) the treatment of boundary conditions, and (3) handling of evaporation/condensation problems. A drawback of the study was that adequate problems related to expected repository conditions were not avallable in the 1iterature. Nevertheless, the results of this study suggest the need for thorough investigations of the impact of heat on the flow field in the vicinity of an unsaturated HLW repository. Recommendations are to develop a new flow code combining the best features of these three codes and eliminating the worst ones.

12. KEY WOROS/DESCR!PTORS (List words or phrases that will assist researchers in locating the report.)

ground-water flow

unsaturated flow

two-phase flow

ground-water modeling

heat-driven flow

coupled processes

high-level radioactive waste disposal

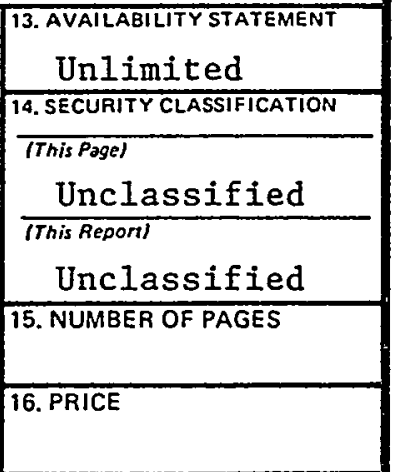

